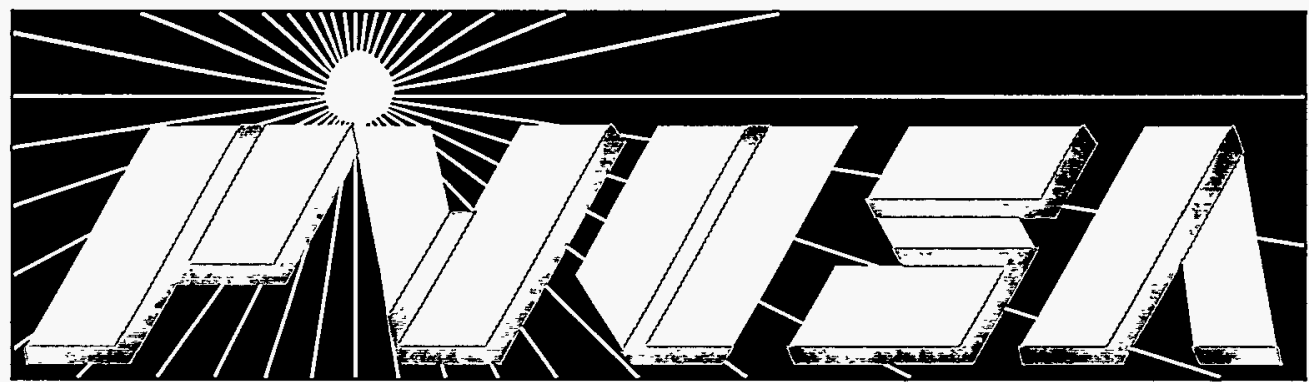

RFCEIVED

JUN 261995

OST

Kerman Photovoltaic Power Plant

R\&D Data Collection Computer System

Operations and Maintenance

June 1994

Prepared by:

P. Bradley Rosen

Endecon Engineering

Project Manager:

Brian K. Farmer 


\section{DISCLAIMER}

This report was prepared as an account of work sponsored by an agency of the United States Government. Neither the United States Government nor any agency thereof, nor any of their employees, makes any warranty, express or implied, or assumes any legal liability or responsibility for the accuracy, completeness, or usefulness of any information, apparatus, product, or process disclosed, or represents that its use would not infringe privately owned rights. Reference herein to any specific commercial product, process, or service by trade name, trademark, manufacturer, or otherwise does not necessarily constitute or imply its endorsement, recommendation, or favoring by the United States Government or any agency thereof. The views and opinions of authors expressed herein do not necessarily state or reflect those of the United States Government or any agency thereof.

\section{KERMAN PHOTOVOLTAIC POWER PLANT R\&D DATA COLLECTION COMPUTER SYSTEM OPERATION AND MAINTENANCE}

DOE/AL/82993-11

Dist. Category UC-1290

June 1994

\section{P. Bradley Rosen}

Endecon Engineering

for

\section{Pacific Gas and Electric Company \\ Research and Development \\ San Ramon, CA 94583}

Published-October 1994

PREPARED UNDER CONTRACT WITH THE UNITED STATES

DEPARTMENT OF ENERGY

Cooperative Agreement No. DE-FC04-92AL82993 


\section{DISCLAIMER}

Portions of this document may be illegible in electronic image products. Images are produced from the best available original document. 


\title{
KERMAN PHOTOVOLTAIC POWER PLANT R\&D DATA COLLECTION COMPUTER SYSTEM OPERATIONS AND MAINTENANCE
}

\section{Registration}

This document is a registered copy if the copy number and assignee below have been completed. Holders of registered copies will receive revisions to this document which may be issued from time to time. To register a copy, change assignee or address, or remove your name from the registration list, contact the PVUSA project office.

\author{
PVUSA Project Office \\ PG\&E Research and Development \\ 3400 Crow Canyon Road \\ San Ramon, CA 94583 \\ Phone: (510) 866-5569 \\ Fax: (510) 866-5447
}

\section{Copy Number:}

Registered To: 


\section{DISCLAIMER}

The Pacific Gas and Electric Company (PG\&E) and the United States Government or any agency thereof, or any of their employees, contractors, subcontractors and their employees, (1) make no representation or warranty, expressed or implied; (2) assume no legal liability for damages, including any consequential damages, even if PG\&E and/or the U.S. Government, or any of their representatives, have been advised of the possibility of such damages; or (3) take no responsibility for the accuracy, completeness, or usefulness of any information, apparatus, product, method, or process disclosed, nor do PG\&E or the U.S. Government represent that its use would not infringe the privately held rights of others. Reference herein to any commercial product, process, or service by trade name, trademark, manufacturer, or otherwise, does not constitute or imply its endorsement, recommendation, or favoring by PG\&E or the U.S. Government, any agency thereof, or any of their contractors or subcontractors.

The work disclosed in this report was done with the support of the U.S. Department of Energy, Cooperative Agreement No.DE-FCO4-92AL82993. However, any opinions, findings, conclusions, or recommendations expressed herein are those of the author(s) and do not necessarily reflect the views of PG\&E or the U.S. Government, any agency thereof, or any of their contractors or subcontractors.

\section{COPYRIGHT NOTICE}

Copyright 1994, PG\&E, subject to the license retained by the U.S. Government under 48 CFR 952,227-75. All other rights reserved. This report and the underlying research were funded and developed for use by PG\&E, members of the PVUSA project, and contributing institutions, including the U.S. Government, the Electric Power Research Institute, and the Califomia Energy Commission. Copies of this report may be obtained for a charge from PG\&E or, if for government use or purposes, from the National Technical Information Service in Springfield, Virginia. Reproduction and/or distribution in any form of this report or any part thereof by anyone other than the U.S. Government is forbidden without express written permission from the copyright owner. 


\section{PROJECT PARTICIPANTS}

In addition to the U.S. Department of Energy (including Sandia National Laboratory, National Renewable Energy Laboratory and Jet Propulsion Laboratory), the Electric Power Research Institute, and the California Energy Commission, the support of all the PVUSA Project members in conducting this research and developing this report is greatly appreciated. Members at the time of publication are:

Central and South West Services, Inc.

City of Austin, Texas

New York State Energy Research and Development Authority

Niagara Mohawk Power Corporation

Public Service Company of Colorado

Sacramento Municipal Utility District, California

Salt River Project

San Diego Gas + Electric

State of Hawaii/Maui Electric Company

U.S. Department of Defense

Virginia Power Company/Commonwealth of Virginia 
Project Manager $\frac{3 \text { niam. Promer }}{\text { Brian K. Farmer }}$

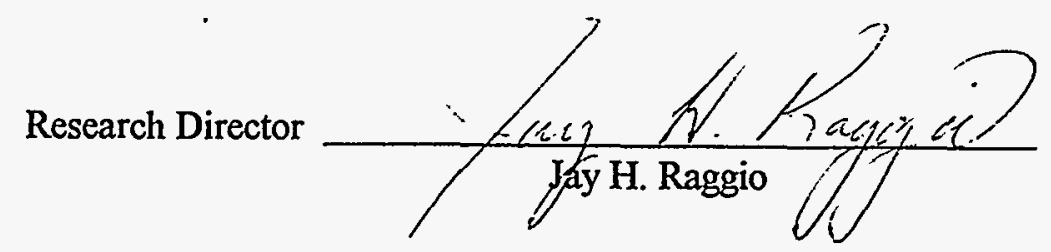




\section{CONTENTS}

\begin{tabular}{|c|c|}
\hline 1 & SYSTEM OVERVIEW ........... \\
\hline \multirow[t]{3}{*}{2} & SCADA \\
\hline & HARDWARE \\
\hline & SOFTWARE \\
\hline \multirow[t]{3}{*}{3} & 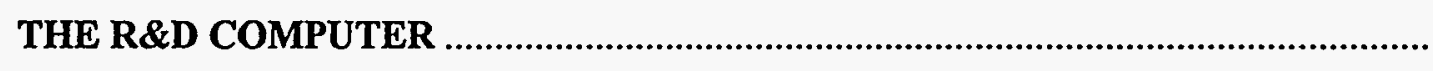 \\
\hline & HARDWARE \\
\hline & 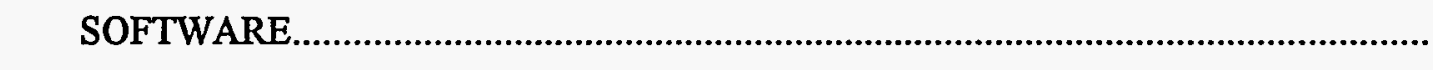 \\
\hline \multirow[t]{2}{*}{4} & 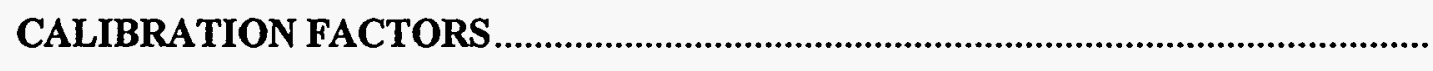 \\
\hline & CHANGING CALIBRATION FACTORS \\
\hline \multirow[t]{2}{*}{5} & THE MODEM AND COMMUNICATION SOFTWARE \\
\hline & THE MODEM PHONE NUMBER \\
\hline 6 & THE TAPE DRIVE AND SOFTWARE \\
\hline 7 & REGULAR MAINTENANCE \\
\hline & HISTORICAL DATABASE MAINTENANCE \\
\hline & 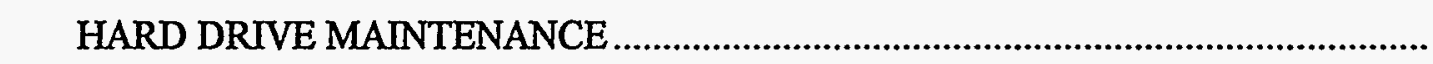 \\
\hline
\end{tabular}

Appendix A:

\section{CABLES}

Appendix B:

\section{PROCEDURES}

Appendix C:

SCADA CONFIGURATION FILES

Appendix D:

SOFTWARE CONFIGURATION FILES

Appendix E:

REALFLEX DATABASE CALIBRATION PARAMETERS

Appendix F:

KERMAN DATABASE MAPPING 
CONTENTS (continued)

Appendix G:

BATCH FILE LISTINGS

Appendix $\mathrm{H}$ :

CONTACTS 


\section{FIGURES}

Figure Page

2-1 Kerman SCADA Hardware Layout..................................................................... 2-1

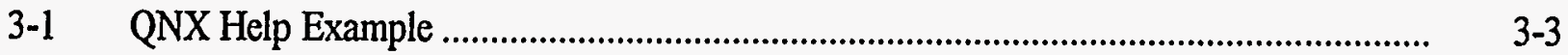

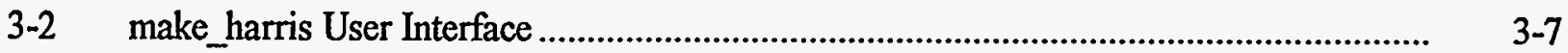

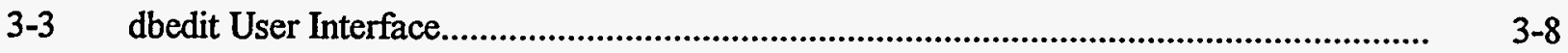

3-4 Line from coldstart File to Load Harris Driver........................................................ 3-9

3-5 Analog Maintenance Screenform................................................................................ 3-10

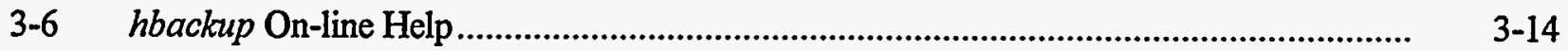

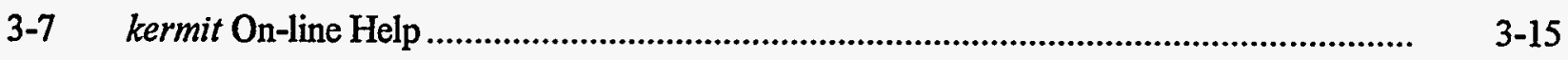

5-1 comm Initialization Line in the coldstart File...................................................... 5-1

7-1 Example Listing of /realflex/data/hist Directory ....................................................... 
TABLES

Tables $\quad$ Page

2-1 SCADA Configuration Files ................................................................................

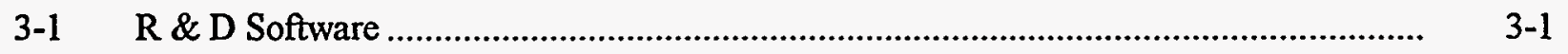

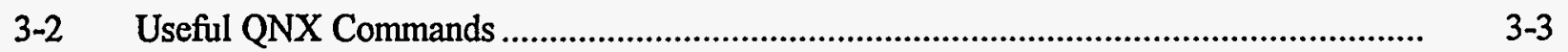

3-3 Harris Port Mapping................................................................................... 3-6

3-4 Kerman DAS Batch Files................................................................................... 3-12

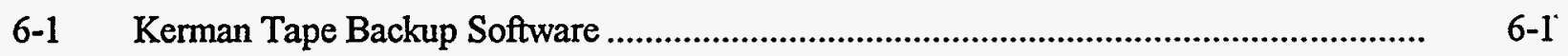




\section{Section 1 \\ SYSTEM OVERVIEW}

The Supervisory Control and Data Acquisition (SCADA) system at the Kerman PV Plant monitors 52 analog, 44 status, 13 control, and 4 accumulator data points in real-time. A Remote Terminal Unit (RTU) polls 7 peripheral data acquisition units that are distributed throughout the plant once every second, and stores all analog, status, and accumulator points that have changed since the last scan. The $R$ \& D Computer, which is connected to the SCADA RTU via a RS-232 serial link, polls the RTU once every 5-7 seconds and records any values that have changed since the last scan. A SCADA software package called RealFlex runs on the R\&D computer and stores all updated data values taken from the RTU, along with a time-stamp for each, in a historical real-time database. From this database, averages of all analog data points and snapshots of all status points are generated every 10 minutes and appended to a daily file. These files are downloaded via modem by PVUSA/Davis staff every day, and the data is placed into the PVUSA database. 
Section 2

\section{SCADA}

\section{HARDWARE}

The SCADA system is comprised of one RTU and seven peripheral units manufactured by Harris/Westronics Inc. This equipment is commonly used by PG\&E for SCADA. The peripheral units include 1 D20A analog input, 1 D20S digital input, and 5 D20C combination boards, all of which are connected via a $250 \mathrm{~Kb}$ transformer-coupled, RS-485 fiber-optic link to the RTU. The peripheral units are used to issue controls, to record analog and accumulator values, and to monitor status (digital) values. These units scan each of their associated channels approximately 1000 times a second, and the RTU scans each of the peripherals once a second (except accumulator inputs which get scanned once every 60 seconds by the RTU).

The RTU has eight RS-232 serial ports (7 data and 1 Westmaint) through which data can be passed to the outside world. At Kerman, two of the data ports are used. Port 1 is used by the PG\&E region to transfer certain data points via radio link to a monitoring center in Fresno, CA using PG\&E serial transfer protocol. Port 5 is used by PG\&E R\&D to transfer all data to the on-site R\&D Computer using Harris protocol. A port on the front panel of the RTU can be used to monitor RTU activity using a built in facility called Westmaint, and 5 data ports on the rear panel are spares.

Figure 2-1 below shows a general overview of the SCADA hardware at Kerman.

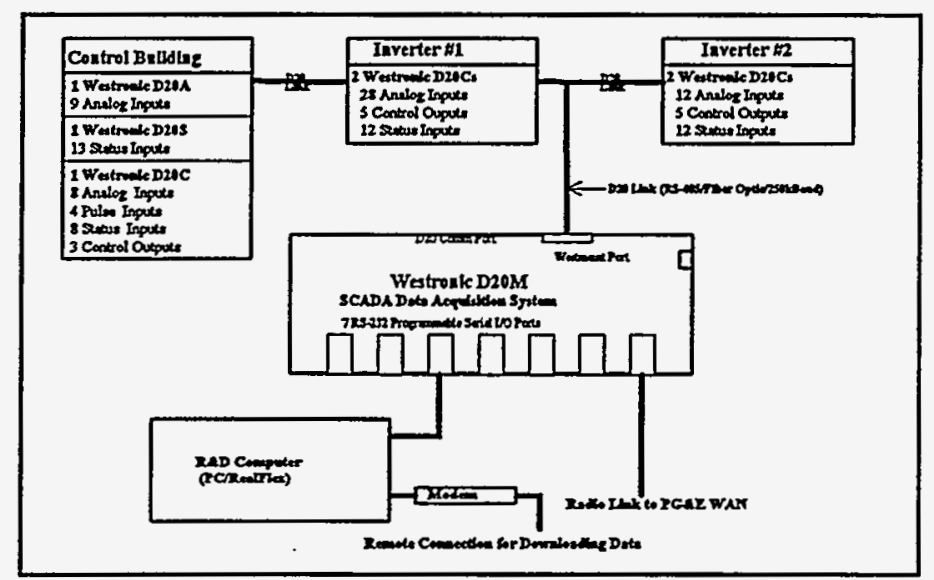

Figure 2-1. Kerman SCADA hardware layout. 


\section{SOFTWARE}

Eight files reside in the RTUs EEPROMs and provide information regarding system configuration and serial protocol information. These files are listed in Table 2-1 along with brief descriptions of their operation.

\section{Table 2-1}

\section{SCADA Configuration Files}

\begin{tabular}{|ll|}
\hline A003.CFG & Harris port configuration file. This file tells the RTU which port to use to transfer data \\
using Harris protocol, what the baud rate will be, serial communication parameters (data \\
bits, stop bits, parity, etc.), what data points to transfer. It also contains the data types, \\
ranges, and count translations for each point in the system. \\
PG\&E protocol configuration file. This file is similar to the A003 file in that it defines \\
which port will be using PG\&E protocol, what data points it will transfer, and other port \\
specific information. \\
DTA configuration file. Comms watchdog file. DTA monitors on-line/off-line status of \\
points specified in this file. Associates specified points with pseudo points specified in \\
this file. \\
B003.CFG \\
D.20 configuration file. This file specifies the different SCADA timing parameters such \\
as analog scan rate, digital scan rate, network retry interval, etc. \\
B012.CFG
\end{tabular}

Software built into the D20 called Westmaint allows a user to interact with the D20 through a nullmodem serial connection (a pin-connection diagram for this cable is shown in Appendix A) from a PC. The Westmaint facility will emulate a VT100 terminal, and provides screens for viewing real-time data of all channels (in count ${ }^{1}$ form), as well as the ability to download new configuration files into the EEPROMs and configure the RTU using its monitor language. Although the user can communicate with the RTU using a standard communications package such as Procomm, a tailored script for CrossTalk IV is available that incorporates the ability to view, manipulate, compile, and download the configuration files, as well as logon to the Westmaint facility.

\footnotetext{
${ }^{1}$ Analog data within the SCADA system is represented in counts where the range for a particular channel can be between -32768 and 32767 . For instance, the standard analog input range is from $-10 \mathrm{~V}$ to $+10 \mathrm{~V}$ (or $+/-1$ Amp with a current shunt installed). So for this example, $-10 \mathrm{~V}$ is represented by -32768 , and $+10 \mathrm{~V}$ is +32767 in the RTU. 
For more information regarding the SCADA hardware and Westmaint, please refer to the Harris/Westronic documentation supplied with the system - a copy is available at the site. 
Section 3

\section{THE R\&D COMPUTER}

\section{HARDWARE}

The R\&D Computer at Kerman is a Dell $486 / 33$ with $8 \mathrm{MB}$ of RAM and a 528MB IDE hard drive. The hard drive has been divided into two partitions, a 105 MB DOS partition and a $423 \mathrm{MB}$ QNX partition. The hard drive can store approximately 14 (all analog channels, 24 hours a day) days of real-time plant data. Beyond that, the integrity of the data and the stability of the system becomes uncertain. The QNX partition is the primary, so the system will always boot into QNX unless the '4' key is held down during the boot process. Other peripherals associated with the R\&D Computer include a 14" Dell monitor, a USRobotics V.32bis Courier modem, and a Tandberg 525MB SCSI tape drive.

The computer is located in the trailer at the site, and communicates with the RTU over an RS-232 serial link. The pin description of the PC/RTU connection cable is described in Appendix A. The R\&D Computer uses COM1 to communicate with the RTU, and COM2 to link to the modem. The computer is powered by an uninterruptable Power Supply (UPS) located in the Control Building.

\section{SOFTWARE}

Table 3-1 lists the primary software used on the R\&D Computer:

\section{Table 3-1}

\section{R\&D Computer Software}

\begin{tabular}{|l|l|c|}
\hline \multicolumn{1}{|c|}{ Name } & \multicolumn{1}{|c|}{ Serial Number } & Version \\
\hline QNX & QNX06950 & 3.20 \\
\hline QNX Windows & N/A & 2.3 \\
\hline Harris Driver & N/A & Dec. 22, 1992 \\
\hline RealFlex Windows & $1220509 \mathrm{~W}$ & 1.22 \\
\hline tdcback & tdc090 & 2.0 \\
\hline Kermit & public domain freeware & \\
\hline
\end{tabular}

Master copies of the software are kept at:

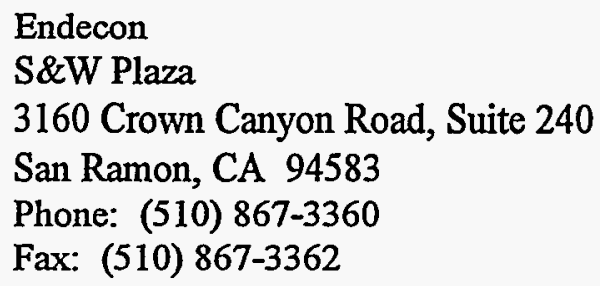


Manuals for the software can be found on site, or at the address shown above.

A tape backup of the hard drive is also kept at the above address, as well as two tape backup copies of all the real-time databases from approximately Junè 1st through September 30th.

\section{QNX/ QNX Windows}

The primary operating system on the R\&D Computer is QNX which is required for operating RealFlex. Like UNIX, QNX is a multi-tasking operating system. Many of the commands are identical to those in UNIX, although the command set as a whole is much smaller. In addition to being a multi-tasking platform, QNX is also a real-time operating system. This means that each process is guaranteed processor time within some maximum time period. That means that no single process can dominate the processor for longer than the maximum time-slice. The net result is that the system is able to handle data in real-time, and all the sub-processes required to process the incoming data can do their respective computations concurrently.

On bootup, the system is configured per commands in the sys.init. 0 file. It then logs in as user 'auto', and starts QNX/Windows and RealFlex automatically. QNX/Windows is simply a graphical, windowing extension of QNX that runs on top of the QNX kernel.

The sys.init.0 file is similar to the config.sys file in DOS. It is basically a QNX script file that has commands to load the video drivers, mount the hard drive and floppy drives, configure the modem and the comm software, configure the comm ports, and to set up passwords and the auto login. A copy of the current sys.init. 0 file can be found in Appendix D, along with descriptions of most of the commands.

As there are too many commands available in QNX to detail here, a few of the standard ones are listed below in Table 3-2. 


\section{Table 3-2}

\section{Useful QNX Commands}

\begin{tabular}{|ll|}
\hline ls & list files in current directory \\
more [fname] & show contents of ASCII file on screen \\
vi [fname] & visual editor - same as vi for UNIX \\
pwd & print working directory \\
cd [directory] & change directory (cd .. goes up one level in the directory structure) \\
tsk & display current operational tasks on screen \\
slay [tsk_name] & remove a task from the system by terminating it \\
zap [file name] & kills a busy file \\
files & gives information about files \\
rm [fname] & remove file \\
mkdir [dimame] & create directory \\
rmdir [dirname] & remove directory \\
\hline
\end{tabular}

Help on any QNX command, although extremely minimal, can be found by typing the command name followed by a question mark. For instance, Figure 3-1 shows how to find more information about the 'files' command.

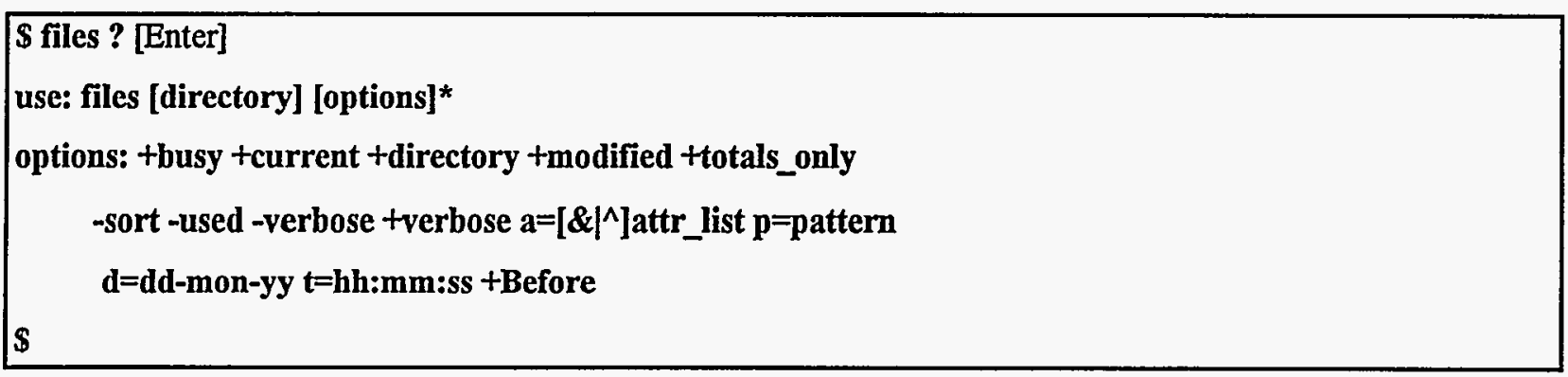

Figure 3-1. QNX help example.

Another important point to note concerning QNX is that drives are represented by numbers, not letters as in DOS. Therefore, the hard drive is device number 3 , and the floppy drives are numbered 1 and 2.

To see a list of all QNX commands, simply change to the /cmds directory by typing cd /cmds [Enter]

Note: A forward slash is used between directories, not a backwards slash as in DOS.

Then type

Is | more [Enter]. 
The "I' symbol means "pipe" the output of $l s$ to the more utility. More simply allows the user to view files one page at a time.

For more information concerning QNX or QNX commands specifics please refer to the QNX Users Manual.

\section{Harris Driver}

The Harris driver is software that "speaks" Harris protocol with the Harris driver on the RTU over the RS-232 line. It asks the RTU for any updated information approximately once every 5-7 seconds. As the real-time data comes in, the Harris driver passes it over to RealFlex for archival in the historical database. The Harris driver is loaded from one of the RealFlex startup files called coldstart which is located in the $3: /$ realflex/data directory. The most important thing about the Harris driver is that it converts RTU counts to Harris counts. The full range for Harris counts is from -2048 to +2047 (integer values). This means that -32768 in RTU counts ( $\max$ negative value on RTU) gets mapped to -2048 Harris counts and +32767 (max positive value on RTU) gets mapped to +2047 . Note that the resolution from the Harris driver is limited to 12 bits versus the 16 bits available.

\section{RealFlex}

RealFlex is a canned SCADA package written by BJ Software in Friendswood, Texas that runs on QNX RealFlex offers facilities to view real-time data, configure, edit, and maintain multiple databases, develop graphical screens and user interfaces for the system, print, interface with QNX, and to accomplish many other SCADA related tasks. A description of the RealFlex configuration at Kerman follows, as well as descriptions of some relevant RealFlex components. For details concerning RealFlex functions please refer to the RealFlex User's Manual.

\section{Startup}

coldstart. The system boots into RealFlex automatically on startup. Configuration information is read from an initialization file called coldstart which resides in the '3:/realflex/data directory'. This file is listed in Appendix D.4. It tells RealFlex what drivers to load, what parameters to use for each of them, and each of their task priorities. coldstart also contains lines to configure printers and defines files to use for scheduled events. A detailed description of this file can be found on page 6-4 in the RealFlex Installation Guide section of the supplied RealFlex documentation. 
rptcron. The QNX scheduler, cron (just as in UNLX), is used by RealFlex to schedule events such as periodic hard drive checks, regular report generation, scheduled batch file execution, tape backups, etc. After completing the driver installation and printer configuration from the coldstart file, RealFlex reads scheduler information from a file called rptcron. This file is listed in Appendix D.2. A detailed description of this file can be found on page 7-2 in the RealFlex Windows Implementation Guide section of the supplied RealFlex documentation. In addition to the default maintenance tasks that are described in the manual, rptcron is used at Kerman to create the daily average data file at midnight and to append the averaged data to this file every 10 minutes. A detailed description of this process can be found on page 3-12.

Manual Startup. Refer to Appendix B.2 for instruction on how to start RealFlex manually from the QNX prompt.

\section{Viewing/Moving Around RealFlex}

The Mouse and Screen. RealFlex has been designed using the Open Look standard. This means that all programs run within a window (or multiple windows). Each window can be closed using its "push-pin" in the upper left-hand comer. The mouse can be used to push buttons, depress push-pins, move windows, cause menus to appear, and make menu selections. The right mouse button is called the "menu button", and the left mouse button is called the "select button". Any selections other than making a menu appear on the screen or making a sub-menu appear from a menu should be made using the left mouse button. All menu selections should be made with the right mouse button. More information concerning the mouse can be found in the RealFlex Windows - Getting Around in RealFlex guide, page 3-1.

Additional explanations of the RealFlex user's interface and functionality of RealFlex can be found in the RealFlex Windows Operator's Guide.

Viewing System Performance and Weather Data. Realflex provides a facility to view the realtime values of all channels in the database. These screens are accessed by choosing 'System Summary Display' from the 'MASTER MENU' on the main RealFlex title bar. This procedure is detailed in Appendix B.12 ("To get basic channel displays from within RealFlex:"). 
Getting to a QNX Shell. This procedure is done using the WORKSPACE menu from within RealFlex Windows. The user is referred Appendix B.9 ("Opening a QNX Window") for instructions on opening a QNX shell from RealFlex Windows.

The Database. Data is mapped from the RTU into the RealFlex database using virtual ports. Virtual ports were originally designed by Harris to mimic the operation of external ports on the older RTUs. Virtual ports have no correspondence to actual I/O ports in the system. They are used in the definition of the database to tell RealFlex where to find each data point in the mapping. The system at Kerman has been designed with 15 virtual ports. The current mapping is shown in Table 3-3. Although each virtual port can map 64 individual channels, the configuration chosen for Kerman allocates 1 virtual port per SCADA peripheral card. This was done to simplify the mapping, and to allow plenty of extra channels for future expansion of the system.

Table 3-3

Harris Port Mapping

\begin{tabular}{|c|l|l|l|c|c|c||}
\hline \hline Port \# & Location & Port Type & Peripheral & Point Range & \# Points & \# CTRL Pts. \\
\hline 1 & Control Building & $\begin{array}{l}\text { Control \& } \\
\text { Indication (1) }\end{array}$ & D20S1 & $1-14$ & 14 & \\
\hline 2 & spare & $\begin{array}{l}\text { Control \& } \\
\text { Indication (1) }\end{array}$ & D20S1 & & & \\
\hline 3 & 12kV Switch & $\begin{array}{l}\text { Control \& } \\
\text { Indication (1) }\end{array}$ & D20C1 & $9-16$ & 8 & 3 \\
\hline 4 & Inverter \#1 & $\begin{array}{l}\text { Control \& } \\
\text { Indication (1) }\end{array}$ & D20C1-INV1 & $1-14$ & 14 & 5 \\
\hline 5 & Inverter \#1 (spare) & $\begin{array}{l}\text { Control \& } \\
\text { Indication (1) }\end{array}$ & D20C2-INV1 & & & \\
\hline 6 & Inverter \#2 & $\begin{array}{l}\text { Control \& } \\
\text { Indication (1) }\end{array}$ & D20C1-INV2 & $1-13$ & 13 & 5 \\
\hline 7 & Inverter \#2 (spare) & $\begin{array}{l}\text { Control \& } \\
\text { Indication (1) }\end{array}$ & D20C2-INV2 & & & \\
\hline 8 & $12 k V$ Switch & Accumulator (7) & D20C1 & $1-4$ & 4 & \\
\hline 9 & spare & & & & & \\
\hline 10 & Weather Station & Analog (3) & D20A1 & $3-10$ & 8 & \\
\hline 10 & Redundancy & Analog (3) & D20A1 & $15-16$ & 2 & \\
\hline 11 & $12 k V$ Switch & Analog (3) & D20C1 & $1-8$ & 8 & \\
\hline 12 & Inverter \#1 & Analog (3) & D20C1-INV1 & $1-15$ & 15 & \\
\hline 13 & Inverter \#1 (spare) & Analog (3) & D20C2-INV1 & $1-5,9-13$ & 10 & \\
\hline 14 & Inverter \#2 & Analog (3) & D20C1-INV2 & $1-12$ & 12 & \\
\hline 15 & Inverter \#2 (spare) & Analog (3) & D20C2-INV2 & & & \\
\hline
\end{tabular}


The information in Table 3-3 is used to create the RTU's database mapping in the A003 file (Harris configuration, Appendix C.1. Consequently, both the Harris driver and the RealFlex database must be made aware of the data mapping as it exists in the RTU. The Harris driver is configured using a program called make_harris.

make_harris. Before a user can define an RTU within the RealFlex database, a template providing the basic port information for the RTU must be created. make harris creates this template. Specifically, make_harris allows the RealFlex database designer to enter the maximum number of channels (data points) that will be used with each virtual port, and what type of data (digital, analog, meter, or accumulator) each port will be mapping, and reports the current status for each port (active $=1$, inactive $=0$ ).

make_harris must be set up before the database can be created and edited, and must be run either from dbedit (refer to Appendix B.3), or in a QNX shell window from RealFlex (refer to Appendix B.9 for the procedure on how to open a QNX window).

Figure 3-2 below shows the make_harris user-interface, as well as the current setup for Kerman.

\begin{tabular}{|c|c|c|c|c|c|c|c|}
\hline \multicolumn{8}{|c|}{$\begin{array}{l}\text { TELEMETRY } \\
\text { Harris Protocol }\end{array}$} \\
\hline \multicolumn{3}{|c|}{ Port Configuration: } & \multicolumn{4}{|c|}{ RTU supports 15 Ports $(\mathrm{Y} / \mathrm{N}) \mathrm{Y}$} & $(\mathrm{N}=7$ Ports! $)$ \\
\hline & type & \# points & $\mathrm{o} / 1$ & & type & \# points & o/l \\
\hline Port 1: & 1 & 32 & 1 & Port 2: & 1 & 32 & 1 \\
\hline Port 3: & 1 & 32 & 1 & Port 4: & 1 & 32 & 1 \\
\hline Port 5: & 1 & 32 & 1 & Port 6: & 1 & 32 & 1 \\
\hline Port 7: & 1 & 32 & 1 & Port 8: & 7 & 4 & 1 \\
\hline Port 9: & & & & Port 10: & 3 & 32 & 1 \\
\hline Port 11: & 3 & 32 & 1 & Port 12: & 3 & 32 & \\
\hline Port 13: & 3 & 32 & 1 & Port 14: & 3 & 32 & \\
\hline Port 15: & 3 & 32 & & & & & \\
\hline
\end{tabular}

Figure 3-2. make_harris user interface. 
In order for RealFlex to collect and convert data from an RTU, it must be told where the data is coming from, how each data point is mapped from the Harris driver's virtual ports, conversion information from Harris counts to engineering units, alarm information, and any other point specific information. This data is entered by the user with a program called dbedit. dbedit must be run from RealFlex. Appendix B.3 gives the procedure for running dbedit.

\section{dbedit}

Because dbedit is a fairly elaborate database interfacing program, the user is referred to section 2 (Database Implementation) on page 2-1 in the RealFlex Windows Implementation Guide, V4. A few of the more confusing aspects of dbedit are detailed here.

The main dbedit screen is shown in Figure 3-3.

PCU MAINTENANCE

NAME ADDRESS ACTIVE CHAN STATUS ANALOGS METERS TANKS

$\begin{array}{llllllll}\text { SYSTEM } & 0 & \text { ON } & 0 & 19 & 2 & 0 & 0 \\ \text { KERMAN_CALIB } & 1 & \text { OFF } & 1 & 0 & 49 & 4 & 0 \\ \text { KERMAN } & 1 & \text { ON } & 1 & 55 & 52 & 4 & 0\end{array}$

F1-ADD F2-DELETE F3-SORT F4-TELEMETRY F5/F6-EDIT DB F7-DISK UTL F8-DB OPTIONS F9-COPY PCU F10-EXIT SF1-WARMSTART SF10-HELP

Figure 3-3. dbedit user interface.

The 'PCU' MAINTENANCE' menu shown in Figure 3-3 is the opening menu of the database editor process (dbedit). Summary information conceming all RTU's in the RealFlex database is input and maintained by the user on this screen. The 'SYSTEM' PCU is internal to RealFlex and keeps information such as printer status, hard drive space available, etc. The 'KERMAN_CALIB' PCU is a special configuration used during calibration, and contains only the analog channels at Kerman. Each point in the 'KERMAN_CALIB' PCU is configured to convert Harris counts back to raw voltages or currents as originally seen by the SCADA peripherals. As can be seen under the 'ACTIVE' column,

2The acronym PCU stands for process control units, and is RealFlex's term for RTU. 
the 'KERMAN_CALIB' PCU is not currently collecting data. The 'KERMAN' PCU represents the normal RTU configuration at Kerman, and contains all the defined data points and calibration coefficients used in the system.

Although the RealFlex database can handle data from multiple RTUs and multiple I/O drivers, the implementation at Kerman is 1 to 1 . This means that there is 1 RTU called 'Kerman', and RealFlex is "fed" data from 1 I/O driver, called the Harris driver. The Harris driver passes data to the RealFlex database (RFDB) through a control queue as defined in both the Harris driver startup line (from within the coldstart file) and dbedit. The harris driver definition from the coldstart file is shown in Figure 3-4. The channel number definition is shown in bold $(c=1)$.

\section{$0 \quad 8 * 10$ harris $\mathrm{c}=10=20=2 \mathrm{~d}=100 \mathrm{f}=2 \mathrm{t}=100 \mathrm{f}=0 \mathrm{p}=\$$ tty $3 \mathrm{~B}=9600$}

Figure 3-4. Line from coldstart file to load harris driver.

In Figure 3-3, the dbedit 'PCU MAINTENANCE' screen, the column 'CHAN' represents the control queues used by each PCU in the system. The ' 1 ' shown for the 'KERMAN' PCU corresponds with the channel number defined for the harris driver as shown in Figure 3-4.

If a new PCU were to be added to the RealFlex database, it would be added on the 'PCU MAINTENANCE' screen shown in Figure 3-3. Before any data points can be added to the system or edited, the "Telemetry Data" for the PCU must be defined. This is done using the make harris program. As explained on Page 3-7, make harris can either be run from within a QNX shell manually, or from dbedit (easiest method). Running make_harris from dbedit simply means pressing the [F4] ('TELEMETRY) key from the 'PCU MAINTENANCE' screen. The phrase "Telemetry" is a term used by RealFlex to refer to any data transferred to and from a remote unit to the RealFlex database over a serial link. Refer to Page 3-7 for more information on using make_harris.

To access the individual point definitions simply use the up/down arrow keys from the 'PCU MAINTENANCE' screen to select the 'KERMAN' PCU, move over to the desired group (ANALOGS, STATUS, etc.) using the [Tab] key, and choose [F6] to edit the database. A screen with 
all the labels for that particular data type will be displayed. Using the arrow key, position the highlighted area over the desired data point and press [Return]. An editing window similar to Figure 3-5 will appear.

\begin{tabular}{|lrlr|}
\hline & \multicolumn{2}{c|}{ ANALOG MAINTENANCE } & \\
TAGNAME: & D20A1_AN03 & HLM: & 0.000 \\
POINT DESC: & WEATHER-GSA & COLOR: & NO ALARM \\
UNITS: & W/m^2 & LLM: & 0.00 \\
POINT OFFSET: & 10 & COLOR: & NO ALARM \\
AUX ADDRESS: & 3 & HHLM: & 0.00 \\
SUBTYPE: & 0 & COLOR: & NO ALARM \\
EU TYPE: & 0 & LLLM: & 0.00 \\
MIN RAW: & -2048 & COLOR: & NO ALARM \\
MAX RAW: & 2047 & ROC TYPE: & 0 \\
MNNEU: & -1800 & ROC INHIIT: & 0 \\
MAX EU: & 1800 & ROC LIMT: & 0.000 \\
& & ALARM ACTION: & 0 \\
& & ALARM & 0 \\
& & DEADBAND: & 677.342 \\
\hline
\end{tabular}

Figure 3-5. Analog maintenance screenform.

Figure 3-5 shows the analog maintenance screenform for the global single axis irradiance data point. The only items that are not either intuitive or explained in the RealFlex users manual are the "POINT OFFSET' and 'AUX ADDRESS'. These fields are used for the Harris virtual port mapping. 'POINT OFFSET' refers to the virtual port \#, and 'AUX ADDRESS' refers to the actual point \# on the associated D20 peripheral unit. Appendix F lists all the points in the system along with their labels, virtual port numbers, actual point numbers, and associated peripherals.

The last important point to note about dbedit concerns notifying the Harris driver following changes to the RealFlex database. This is done by typing [Shift]-[F1] ('WARMSTART') from the 'PCU MAINTENANCE' screen. The Harris driver should be "warm-started" in this fashion whenever any changes are made using dbedit.

\section{histdb}

RealFlex communicates with the Harris driver to obtain any updated data from the RTU, and puts it into the historical database using a process called histdb. histdb is the database manager. All inquires from the database and inserts into the database must go through histdb. histdb converts from Harris 
counts $(-2048$ to +2047$)$ to engineering units before placing the data in the database. The conversion parameters are defined for each point when the database is configured using dbedit (with the 'MIN/MAX RAW' and 'MIN/MAX EU' fields). All calibration factors for the system are incorporated into the conversion parameters for each point. Calibration factors are discussed on Page 4-1, and are listed in Appendix E.

Some important points concerning histdb:

- histdb must be running for any program, driver, or user to interface with the database in any way.

- The RealFlex database files can not be manually accessed, moved, renamed, or deleted when histdb is running. Refer to Appendix B for procedures concerning the database files.

- histdb is responsible for all calculations pertaining to data in the database when accessed through RealFlex. This is pertinent to the generation of averaged data for the PVUSA database because it is important to know that histdb is generating the averages and not the Report Generator.

- histdb should always run at priority level 7. This is one higher than normal, and therefore requires a ' $t$ ' sign following the ' $\&$ ' whenever starting histdb manually. For example, after deleting the real-time database the user would restart histdb by typing "ontty \$tty0 histdb \&+[Return]". As an aside, the "ontty \$tty0 histdb" command tells the system to start histdb on virtual console 0 (as opposed to the current console) where all the other system processes exist. We do this so that the process will not be killed when we logoff, or if some problem arises with the modem (the process for which exists on \$tty4).

The Report Generator. The Report Generator is a RealFlex utility that can be used to assemble "reports" based on data in the historical database. The user can use The Report Generator to create custom reports that include averages, mins, maxs, raw data, etc. The procedure for running The Report Generator from RealFlex is shown in Appendix B.4. For particulars concerning The Report Generator the user is referred to page 5-1 of the RealFlex Windows Implementation Guide, V4.

The Report Generator is used at Kerman to produce the 10-minute averages in comma-separated-value (.CSV) format. The name of the report is 'Kerman'. The report contains field definitions for every analog, status, and accumulator point in the system. Because The Report Generator only accepts a certain number of characters per line, and because the field definitions for all the points at the plant take up much more space than is available on one line, the .CSV format was set up in multiple lines. Each field is separated by 
a comma in the report so that when the 'Kerman' report is generated every 10 minutes, each data point is separated by a comma in the output file. Each of the analog fields in the 'Kerman' report has been defined to output the average for that particular point over the previous 10 minutes. The first field on the first line contains the date and time the report was generated. This date/time field is generated from the system clock.

In order to make the data available any time during the day, to keep the time it takes to generate a report to a minimum, and to get the daily files in a semi-manageable form, the 'Kerman' report is generated once every 10 minutes instead of once a day. rg_exec (the Report Generator executable file) is spawned every 10 minutes by cron (as specified in rptcron). The Report Generator executes the 'Kerman' report, generating averages for the previous 10 minutes (analog values only, status values are snapshot). The report is output to the file 3:/realflex/bin/Kerman. 3 minutes following each generation of the 'Kerman' report (i.e. 10:13, 10:23, 10:33, etc.), cron spawns a batch file (refer to section on batch files) which appends the 'Kerman' report file to a file in the 3:/realflex/data/Kerman.data directory of the format "DDMMYYkm.csv" that contains all of the 10-minute averages since midnight on that day.

\section{Batch Files and Daily Average File Generation}

As described previously, QNX batch files are used to create a new daily file at midnight every night, and to append the average data reports generated every 10 minutes to this file. These batch files are explained briefly in table 3-4, and are listed in Appendix G.

Table 3-4

\section{Kerman DAS Batch Files}

\begin{tabular}{|l|l|l|}
\hline \multicolumn{1}{|c|}{ Name } & \multicolumn{1}{|c|}{ Directory } & \multicolumn{1}{c|}{ Description } \\
\hline rundaily.bat & /realflex/data/Kerman.batch & $\begin{array}{l}\text { Creates temporary file with current date in } \\
\text { it, then uses led and ledcmds to parse date. }\end{array}$ \\
\hline ledcmds & /realflex/data/Kerman.batch - & $\begin{array}{l}\text { Contains led commands to parse date and } \\
\text { create the run10.bat file. This file is } \\
\text { passed to led by rundaily.bat. }\end{array}$ \\
\hline run10.bat & /realflex/data/Kerman.batch & $\begin{array}{l}\text { This file is run after each 10-minute } \\
\text { average report is generated. It appends } \\
\text { Kerman report file to a daily file of the } \\
\text { name DDMMYYkm.csv. }\end{array}$ \\
\hline
\end{tabular}


rundaily.bat. The process of appending 10-minute average data to a daily file begins at 12:04am every night. The file rundaily.bat is spawned by cron (according to rptcron) at this time. rundaily.bat uses the system date command to put the current date and time into a file called datefile in the 3:/realflex/data/Kerman.batch directory. Then, rundaily.bat uses the system led command to parse the date in datefile and create the runI0.bat file according to the commands that are redirected (with the ' $<$ c symbol) to it from the file ledcmds.

ledcmds. ledomds is a command file that is passed to the system led command by rundaily.bat. It first parses out the date from the date/time string in datefile. An example string is shown below.

24-Nov-93 12:05:00 am

ledcmds parses the string taking the dashes out of the string, replacing the character representation of the month with the numerical (ie. 11), and removing the time. So, the string looks like

\section{3}

after the first stage of ledcmds. Next, ledcmds appends 'km.csv' to the string forming

$241193 \mathrm{~km} . c s v$

Now that the daily file string has been created, ledcmds instructs led to put the system concatenate command (cat) followed by the path that the file will reside in before the string as follows:

cat 3:/realflex/data/Kerman.data/241193km.csv

The form of the cat command is cat [input_file1] [input_file2]* > [output_file]. Because the cat command will not create a new file if the output file does not exist, the report file must be concatenated to the daily file and the result must be stored in a temporary file [tmp]. Then the temporary file must be copied over to the daily file (this will overwrite the previous daily file each time, or will create a new daily file with the first report in it at 12:10am). ledcmds does this by continuing the previously described process and assembling the following two lines:

- cat 3:/realflex/data/Kerman.data/241193km.csv 3:/realflex/bin/Kerman >

- $3: /$ realflex/data/Kerman.batch/tmp

- cp 3:/realflex/data/Kerman.batch/tmp 3:/realflex/data/Kerman.data/241193km.csv 
These lines are then written to a file called run10.bat and that file is made executable with the command (last line in rundaily.bat)

$$
\text { chattr } a=+e \text { rundaily.bat }
$$

Now the file run10.bat exists with the commands to append the Kerman report to an existing daily file in the /realflex/data/Kerman.data directory, or if it is the first report of the day and no daily file exists yet, runl0.bat will create the daily file with the first 10-minute average report in it.

Once runl0.bat has been created at 12:04am each day, it can be run after each 10-minute report is generated and the new data will be appended to the daily file.

\section{hbackup}

hbackup is a RealFlex utility found in the realflex/bin directory, used to backup a realflex database to an ASCII file, or restore an ASCII file previously backed up using hbackup. hbackup allows the user to specify an end time, duration, what calculations (average, min, max, raw) histdb should perform on the data (compression), the compression interval, and what points to include. hbackup is particularly useful when " 5 -second" data is needed in a manageable form. It provides the only method for transferring "5second" data to a readable ASCII format. Figure 3-6 is a screen dump of the on-line help for hbackup and shows the format used.

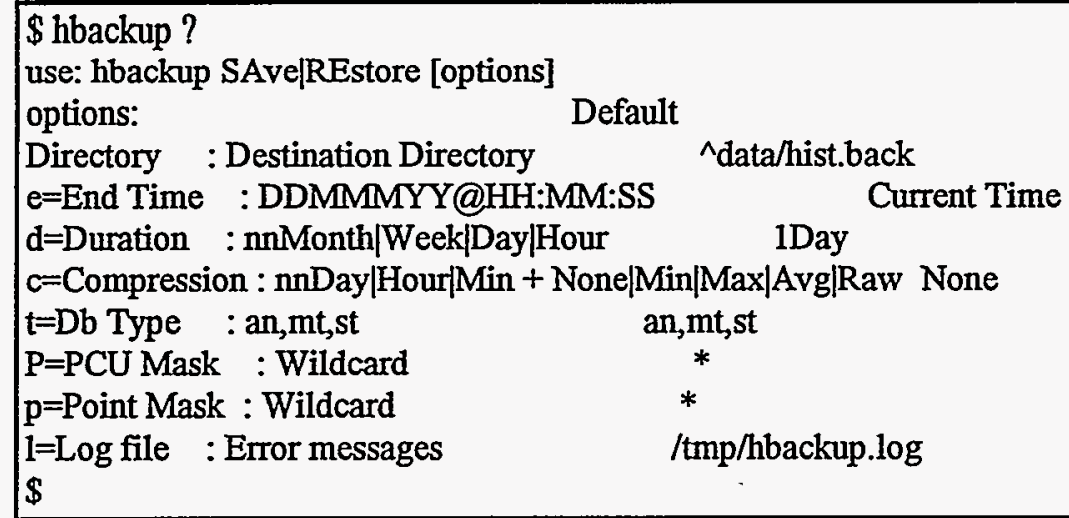

Figure 3-6. hbackup on-line help.

For example, if the user wanted to write the raw analog " 5 -second" data to a file for the period between 12:00:00 and 15:00:00 on October 15, 1993, he would type 
at the QNX prompt while RealFlex was running. RealFlex must be running to use hbackup. Unless a specific directory is specified as the first parameter on the command line, the data new data file will be placed in the /realflex/data/hist.back directory. The file is always named using the date of the last data point in the sample. Therefore, the name of the data file for this example would be

\section{$150 \mathrm{ct} 93 . ? ?$}

and will be found in the realflex/data/hist.back directory.

\section{kermit}

kermit is a standard serial communications file transfer protocol. It is installed on the Kerman R\&D Computer. kermit was chosen because it is a standard protocol that has been implemented on most platforms, and because it is one of the few offered for QNX. Because kermit is the only serial transfer protocol on the system at Kerman, it is used for all transfer of files to and from the R\&D Computer by both serial and direct links through the comm port. The kermit on-line help is shown in Figure 3-7.

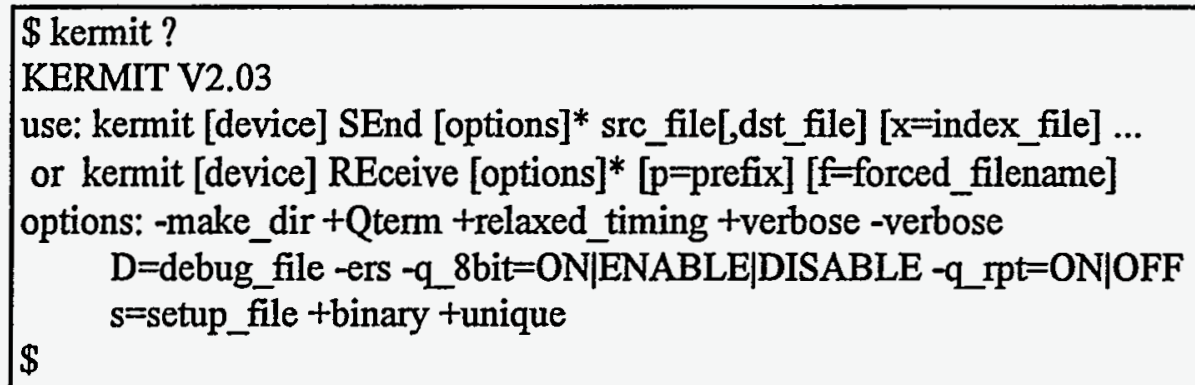

Figure 3-7. kermit on-line help.

A typical use for kermit at Kerman would be to download a daily 10-minute average data file. This is done by logging on to the system via modem (refer to Appendix B for logging on procedures) and typing the following line at the QNX prompt:

$$
\text { kermit se [data_file_name] }
$$

where [data_file_name] is replaced by the actual name of the file to download. The user's remote communications program should automatically initiate the transfer procedure. If the remote comm program does not automatically start the download, the user should manually initiate the download specifying kermit as the protocol. 
Section 4

\section{CALIBRATION FACTORS}

Calibration factors were determined in April of 1993 for all analog points at Kerman. During calibration, known values (voltages or currents) were input into the system at the various measurement points throughout the plant. These values were held constant for five minutes per input level, and were then increased to the next level. During this process the RealFlex system recorded "5-second" data for all analog channels. Following the completion of each channel, 1-minute averages were generated using the Report Generator. These averages were then imported into Excel and regressed against the actual values that were injected into the system. A multiplier for each channel was calculated based on the regressions performed. The multipliers for each channel were then applied to the Ymin and Ymax values in the database using dbedit. Appendix $\mathrm{E}$ lists all analog $\mathrm{Ymin}$ and $\mathrm{Ymax}$ values in the Kerman database.

For more information concerning calibration techniques, procedures, and actual regression values, please refer to Appendix $\mathrm{H}$ for contact names and telephone numbers.

\section{CHANGING CALIBRATION FACTORS}

Follow the procedure called 'Running the Database Editor (dbedit)' (Appendix B.3). Move the cursor to the 'KERMAN' PCU using the down arrow keys. Next, using the [Tab] key, move the cursor to the 'ANALOGS' column and press [F6] ('EDIT DB') to edit the database. A window with all the analog scan points in the system should come up listed by tag name. By positioning the cursor on the particular point whose calibration constants you want to change and pressing [Return], a window with all the individual configuration information for that point should come up. To change the calibration constants, simply change the appropriate 'YMAX', 'YMIN', 'EUMAX', and 'EUMIN' fields as desired. Before exiting make sure to do a 'WARMSTART' by pressing [Shift]-[F1] from the main dbedit screen. 


\section{Section 5 \\ THE MODEM AND COMMUNICATION SOFTWARE}

The R\&D Computer at Kerman is set up with a USRobotics V.32bis modem connected to COM2. The modem is a 14.4 kbaud modem capable of baud rates up to 19200 bits per second with compression. Upon startup of the system, the modem is initialized by a program called comm from within the sys.init. 0 file. comm runs in the background on the computer and emulates a virtual terminal through the modem. This enables other computers to connect to the Kerman R\&D Computer via modem, and login as a new user as if directly connected on a LAN. PLANT CONTROL CAN NOT BE PERFORMED REMOTELY! To get a password, contact the PVUSA project manager at the number shown in Appendix H. The comm initialization line in sys.init. 0 is shown in Figure 5-1.

ontty \$tty4 comm $+1+h$ b=19200 $i=a t \& f \& a 3 \& b 1 \& h 1 \& r 2|a=A T A| l=\$ c o n l ~ q=15$ "m=Welcome to the Kerman R\&D

Computer"

Figure 5-1. comm initialization line in the coldstart file.

The initialization line shown in Figure 5-1 starts up comm on \$tty4, which is QNX's name for COM2. We use COM2 because COM1 is being used by RealFlex to talk to the RTU. The baud rate is set at 19200 by setting " $\mathrm{b}=19200$ ", and the modem is initialized with the string following the " $\mathrm{i}=$ ". The " $a=$ " parameter is the modem's manual answer string, and is sent to the modem by comm when a ring alert is sent to the computer from the modem. The other parameters can be referenced in the QNX Users Manual.

\section{THE MODEM PHONE NUMBER}

The phone number for the line that the modem is connected to is:

(209) 846-7958 
Section 6

\section{THE TAPE DRIVE AND SOFTWARE}

A 525MB SCSI Tandberg tape drive is connected to the Kerman R\&D Computer. The software required to use the tape drive is located in the 3:/tdcback directory. These files are listed and described in Table 6-1.

Table 6-1

Kerman Tape Backup Software

\begin{tabular}{|c|c|c|c|}
\hline File & Directory & Page & Description \\
\hline$a h a$ & /tdcback & 7 & $\begin{array}{l}\text { SCSI driver for tape - must be loaded before tdcback, tdc, or } \\
\text { tdcdir is used. }\end{array}$ \\
\hline tdcback & /tdcback & 13 & Main backup software. Saves, restores, lists files of tape, etc. \\
\hline$t d c$ & /tdcback & 12 & $\begin{array}{l}\text { Small utility program to gracefully unload aha, and to send } \\
\text { SCSI commands to devices on the SCSI bus. }\end{array}$ \\
\hline tdcdir & /tdcback & 22 & $\begin{array}{l}\text { Utility for saving and restoring a directory tree. } t d c d i r \text { is not a } \\
\text { client of the } a h a \text { administrator as are the other tdc programs. }\end{array}$ \\
\hline TDCBACK_FULL & /cmds & N/A & $\begin{array}{l}\text { Batch file that automatically loads } a \text { ha and does a full backup } \\
\text { to tape of the entire hard disk. Any errors go to } \\
\text { /tdcback/errors, and a verify log is written to } \\
\text { /tdcback/verify_log. Gracefully unloads aha when finished. }\end{array}$ \\
\hline$T D C B A C K \_I N C R$ & /cmds & N/A & $\begin{array}{l}\text { Batch file that automatically loads aha and does an } \\
\text { incremental backup of the entire hard disk. Any errors go to } \\
\text { /tdcback/errors, and a verify log is written to } \\
\text { /tdcback/verify_log. Gracefully unloads aha when finished. }\end{array}$ \\
\hline$T D C B A C K \_C S V$ & /cmds & N/A & $\begin{array}{l}\text { Batch file that automatically loads aha and does a backup of } \\
\text { the /realflex/data/Kerman.data directory (not subdirectories). } \\
\text { This batch file is intended to back up all the daily data files. } \\
\text { Any errors go to /tdcback/errors, and a verify log is written to } \\
\text { /tdcback/verify_log. Gracefully unloads aha when finished. }\end{array}$ \\
\hline TDCBACK_HIST & /cmds & N/A & $\begin{array}{l}\text { Batch file that automatically loads aha and does a backup of } \\
\text { the /realflex/data/hist.save directory where the backed up } \\
\text { historical databases reside. Any errors go to /tdcback/errors, } \\
\text { and a verify log is written to /tdcback/verify_log. Gracefully } \\
\text { unloads aha when finished. }\end{array}$ \\
\hline TDCBACK_LIST & /cmds & N/A & $\begin{array}{l}\text { Batch file that automatically load aha and lists all the files on } \\
\text { the tape currently in the drive. Any errors go to } \\
\text { /tdcback/errors, and the actual list is written to /tdcback/list. }\end{array}$ \\
\hline
\end{tabular}

The last five items in Table 6-1 list batch files that have been developed for some of the common backups done regularly on the system. These files may be run from any directory. Listings of these files can be found in Appendix $\mathrm{G}$, and explanations of the various commands used are contained in the tdcback manual. 
In general, these tape backup procedures take a long time. When executed remotely they should be run on virtual console \$tty0, which is the system console, so the user doesn't have to be logged in for the entire duration of the backup. To run one of the batch files when logged on remotely, simply type:

\section{ontty \$tty0 [batch file name] [Return]}

After starting the batch file with the command above, the user may log out of the system, and the backup will proceed until finished or internupted by an error. The batch files listed in Table 6-1 put an error log called errors and a verify log called verify_log in the /tdcback directory. It is in the user's best interest to consult these logs after each backup is complete to confirm that all went as planned.

New tapes must be formatted before any data can be stored on them. The procedure 'Initializing a Tape' in Appendix B will format and initialize a new tape. Formatting a tape erases all data that was previously stored on it. 
Section 7

\section{REGULAR MAINTENANCE}

\section{HISTORICAL DATABASE MAINTENANCE}

Because the RealFlex historical database is set up to collect data every 5-7 seconds on all analog, status, and accumulator channels, it gets quite unwieldy in size very quickly. As the database approaches $65 \mathrm{MB}$ the system becomes unstable, averages get lost, and the directory and file structure on the hard disk become corrupt. Therefore, it is important to monitor the size of the historical database frequently.

The RealFlex historical database and its associated files are kept in the

\section{/realflex/data/hist}

directory. The file that contains all of the data, and that is of concem, is called

\section{histdata}

By changing to the /realflex/data/hist directory with the command

$$
\text { cd /realflex/data/hist [Retum] }
$$

and using the files command, the sizes, dates and times last changed, and file names of all historical data files will be display as shown in Figure 7-1.

\begin{tabular}{|llll|}
\hline files & & & \\
& & & \\
Blks & Date & Time & Name \\
15 & $1-$ Dec-93 & $9: 15 \mathrm{am}$ & data.bit \\
60552 & $1-$ Dec-93 & $9: 04 \mathrm{am}$ & histdata \\
1676 & $1-$ Dec-93 & $8: 22 \mathrm{am}$ & histindex \\
1 & $1-$ Dec-93 & $9: 15 \mathrm{am}$ & index.bit \\
5 & $1-$ Dec-93 & $8: 00 \mathrm{am}$ & index.root \\
& & & \\
Total blocks: 62249 & Total files: 5 & & \\
$\$$ & & & \\
\hline
\end{tabular}

Figure 7-1. Example listing of /realflex/data/hist directory. 
Notice the line shown in bold in Figure 7-1. The number ' 60552 ' is the size of histdata in blocks. One block equals 512 bytes. Therefore, the size of histdata shown in Figure 7-1 is

$$
60552 * 512=31,002,624 \text { bytes (31 Mbytes). }
$$

When the size of this file exceeds 100,000 blocks ( 50 MB), the procedure for 'Removing (or Moving) the Real-Time Historical Database' shown in Appendix B should be followed. Under no circumstances should the size of histdata exceed 127,000 ( $65 \mathrm{MB})$ blocks! The size of histdata should be checked every time a user logs onto the system. Generally, it should be removed once every two weeks.

During the summer months when data are critical, all of the real-time data should be archived to two tapes. This procedure starts by moving each historical database as it gets too large every two weeks into its own temporary directory. Then, once a month, the temporary directories containing the old historical databases should be backed up using the command TDCBACK_HIST. Before deleting the old databases, there should be a copy of each on TWO different tapes. So, during the time period when all the data are being saved, and the histdata file gets to a size where it should be removed, follow the procedure for 'Moving the Real-Time Historical Database'.

It is important to note that these files can not be manipulated in any way while histdb is running. histdb is the database manager, and locks these files when it is active. Whenever the historical database is to be backed up, moved, or deleted, the histdb process must be slayed first, and then restarted on $\$$ tty 0 with the ' $\&+$ ' parameter when done.

\section{HARD DRIVE MAINTENANCE}

Occasionally, when the system gets bogged down because of a database that has gotten too large, or when an operation gets canceled before it has completed writing to disk, the integrity of the directory and file structure on the hard disk gets "confused". It is therefore good practice to perform regular hard drive maintenance operations that check for any abnormalities on the hard drive. Unfortunately, these procedures must be done at the site. The procedure "Hard Drive Maintenance" on Appendix B.6 should be carried out at least once every two months. 
Additionally, it is important to monitor the free space on the hard drive frequently, and to delete unneeded files whenever possible. The command to quickly see how much hard drive space is available is query [Return] 
Appendix A

\section{CABLES}

0075947IDSS2 


\section{CABLES}

A.1. RealFlex PC-to-RTU Cable Pin Connections

\begin{tabular}{|l|l|l|l|l|}
\hline X-END SIGNAL (D20) & PIN NO. & COLOR CODE & PIN NO. & Y-END SIGNAL (RF) \\
\hline RTS & 7 & RED & 1 & DCD \\
CTS & 8 & & & \\
$+\mathrm{V}(5 / 12)$ & 4 & & & \\
\hline RXD & 2 & WHT & 3 & TXD \\
\hline TXD & 3 & GRN & 2 & RXD \\
\hline COM & 5 & BLK & 5 & COM \\
\hline DCD & 1 & ORN & 6 & $\begin{array}{l}\text { RSR } \\
\text { RTS } \\
\text { CTS }\end{array}$ \\
\hline
\end{tabular}


Appendix B

\section{PROCEDURES}

D075947/DSS2 


\section{PROCEDURES}

\section{B.1. Booting into DOS}

To boot into the DOS partition on the R\&D Computer simply press the ' 4 ' key down after the memory check and before the computer beeps.

\section{B.2. Running RealFlex from the QNX Prompt}

From the QNX prompt type:

$$
\text { windows [Enter] }
$$

Then, when the mouse pointer becomes visible, position the mouse pointer anywhere on the screen other than the menu bar on top and click the right mouse button

A menu should appear at the mouse pointer. Position the mouse pointer over 'Programs' and click with the right mouse button.

Another menu should appear to the right of the first menu. Position the cursor over the word 'RealFlex' and click with the left mouse button.

RealFlex should now start. It will wait about 5 seconds for you to choose 'WARM START' or 'COLD START', but will then continue on to do a 'WARM START' by default.

\section{B.3. Running the Database Editor (dbedit)}

1. Position the mouse cursor over the 'MASTER MENU' button on the RealFlex menu bar and click on the left mouse button.

2. Position the mouse cursor over the 'UTMITIES' option on the menu that appears below 'MASTER MENU' and click with the left mouse button.

3. Choose 'DATABASE EDITOR' in the upper-left hand comer of the next menu that appears to the right of 'Utilities' and click on the right mouse button. dbedit should come up.

\section{B.4. Starting the Report Generator}

1. Position the mouse cursor over the 'MASTER MENU' button on the RealFlex menu bar and click on the left mouse button.

2. Position the mouse cursor over the 'UTILITIES' option on the menu that appears below 'MASTER MENU' and click with the left mouse button.

3. Position the mouse cursor over the 'REPORT GENERATOR' and click with the left mouse button.

\section{B.5. Shutting RealFlex Down}

1. Position the mouse cursor over the 'MASTER MENU' button on the RealFlex menu bar and click on the left mouse button.

2. Position the mouse cursor over the 'UTILITIES' option on the menu that appears below 'MASTER MENU' and click with the left mouse button.

3. Position the mouse cursor over the 'EXIT REALFLEX' and click with the left mouse button. 
4. A message box will appear asking if you are sure you want to exit RealFlex. Click on 'YES' with the left mouse button.

5. Press [Return] after the message to type 'windows' appears in text.

\section{B.6. Hard Drive Maintenance}

You must be at a QNX prompt to execute this procedure. If Realflex is running, exit it using the 'Shutting Realflex Down' procedure. At the QNX prompt type:

slay comm $t=4 u=8000-v-q$ [Return]

slay olwm [Return]

slay dialog [Retum]

slay screen_event [Return]

slay queue [Return]

Next, type tsk followed by [Return] and verify that 12 or less tasks are running on the system.

Then type dcheck 3 [Return]

Press [Return] when it prompts you. When it is finished, type

files 3:/ t $b$ [Return] and make sure no files are listed as busy. If files are busy, at the prompt type: zap filename. Then type

chlfsys 3 [Return] and yes [Return] after you have read the warning. If any errors come up follow the instructions to have the program fix them.

Reboot the computer using the reset button on the front panel when chkfsys is finished. All these were done to make sure $\mathrm{HD}$ is in good condition.

\section{B.7. Initializing a Tape}

From the QNX prompt type the following lines:

cd /tdcback [Return]

aha \& [Return]

tdcback aha0 1 init "put_file_name_here" [Return] (example: Kerman_backup_tape\#X, where $\mathrm{X}$ is a number greater than last one. Check directory.)

tdcback aha0 1 quit [Return]

\section{B.8. Tape Backup Procedures}

\section{B.8.1. Backing Up the Hard Drive While at the Site}

Before you start follow Hard Drive Maintenance Procedures above.

1. Make sure the desired tape is in the tape drive. (Initialize, if necessary or read the TapeX.list file for important information about the Tape.)

2. Although RealFlex can be running when a backup is done, it is suggested that it be shut down so that all files on the hard disk are accessible. RealFlex locks certain files and therefore will not allow them to be backed up while it is running. To shut RealFlex down follow the procedure for 'Shutting RealFlex Down'. 
3. To initiate the backup, pick the appropriate batch file from Table 6-1, type its name in at the QNX prompt followed by a [Return]. Make sure to use capitols. For example, to initiate a full backup of the hard drive type

\section{TDCBACK_FULL [Return]}

at the QNX prompt. The backup should commence and it takes about 2 hours.

4. Make list of changed tapes. From the QNX prompt type the following lines:

cd/tdcback [Return]

aha \& [Return]

tdcback aha0 1 list $>$ tapeX.list [Return]

mv tapeX.list/realflex/data/kerman.data/tape_backup_logs [Return]

The files errors, tapeX.list, and verify_log will contain pertinent information concerning the backup after it is completed. The files can be found in the /tdcback directory.

5. more Verify_Log file to make sure backup was successful and move file to tapes_logs directory.

\section{B.8.2. Backing Up the Hard Drive Remotely}

For this procedure the user must be logged in to the Kerman system remotely. This procedure will not back up the /realflex/data/hist directory (namely the current historical database) because RealFlex is running (unless histdb is slayed). So, follow the procedure for 'Shutting Down $h i s t d b$ ' or 'Removing the Real-Time Database' prior to executing this procedure if the current RTDB should be backed up.

1. Shut down histdb if necessary.

2. Choose the appropriate backup procedure from Table 6-1.

3. Type the following line at the $\mathrm{QNX}$ prompt, replacing TDCBACK_FULL with the appropriate batch file name.

\section{ontty Stty0 TDCBACK_FULL [Return]}

4. The backup should commence. The files errors and verify_log will contain pertinent information concerning the backup after it is completed. The files can be found in the /tdcback directory.

5. Log off system as desired, the backup will continue in the background until it is finished, or it encounters an unrecoverable error.

\section{B.9. Opening a QNX Window}

1. Position the mouse cursor in a blank area of the screen (the RealFlex base window) where no windows are open, and no buttons or menu bars exist (in other words, on the background of the screen).

2. Click with the right mouse button. A menu should appear.

3. Position the mouse cursor over the 'UTILITIES' option and click with the right mouse button. A new menu should appear just to the right of the previous one.

4. Position the mouse cursor over the 'SHELL' option and click with the left mouse button.

4. A QNX window should open. To close this window type 'exit' from the QNX prompt within this window.

MAKE SURE TO CLOSE THE QNX SHELL WINDOW WHEN FINISHED! 


\section{B.10. Direct serial link between R\&D Computer and another PC}

1. Make sure you have a null modem connection from laptop (COM1) to R\&D Computer (COM2)

2. Run any comm program you want on laptop (like term.exe in windows) with the following settings:
a. baud $=19200$
b. direct connection
c. hardware flow control

3. On R\&D Computer, get into a QNX shell by clicking the right mouse button on the windows background, choosing utilities from the menu, and choosing shell from the following menu.

4. type:

slay comm $t=4 u=8000-v-q$

stty + fix $>\$$ tty 4

stty inton $=3$ baud $=19200$ thangup $>\$$ tty 4

ontty $\$$ tty 4 login

The laptop should be asking for login at this time. Download as desired.

\section{B.11. Procedure for re-connecting modem to R\&D Computer.}

0. Connect modem to COM2 (direct serial connection)

1. Cycle power on modem

2. In QNX shell type:

slay login $t=4$

stty + fix $>\$$ tty 4

stty inton $=3$ baud $=19200$ bits $=8$ par $=$ none stop $=1$ esc $=0$ thangup up=al down=a9 right=a6

left $=\mathrm{a} 4$ ins $=\mathrm{ab}$ del $=\mathrm{ac}>\$ \mathrm{tty} 4$ DAS"

ontty $\$$ tty 4 comm $+1+h b=19200 i=a t \& f|" a t| a=a t a ~ l=\$ c o n 1 q=15$ "m=Welcome to the Kerman

Notice where the quotes are on the " $m="$ line, it's important!

\section{B.12. To get basic channel displays from within RealFlex:}

1. Click on MASTER MENU with the right mouse button

2. Click on SYSTEM SUMMARY with the right mouse button

3. Click on KERMAN with the right mouse button

4. Click on PAGE DOWN and PAGE UP with right button to scroll through analog, status, control, and accumulator data points.

5. To close: Click on "push-pin" in upper left corner of window with left mouse button.

\section{B.14. Removing the Real-Time Historical Database:}

1. From the QNX prompt type 


$$
\text { cd/realflex/bin[Retum] }
$$

2. Type

$$
\text { pwd[Return] }
$$

and verify that you are now in the /realflex/bin directory.

3. Type

$$
\begin{aligned}
& \text { start_up +t[Return] } \\
& \text { slay Wrealflex [Return] } \\
& \text { cd /realflex/data/hist[Retum] } \\
& \text { Is[return] }
\end{aligned}
$$

verify that the file 'histdata' is in that direcotory

2. Type

The next line will DELETE the real-time database files - if they need to be archived first, follow the procedure for Moving the Real-Time Historical Database.

To delete the database type:

$$
\text { rm *[Return] }
$$

3. Type

$$
\begin{aligned}
& \text { cd /realflex/bin[Return] } \\
& \text { ontty \$tty0 realflexRetum] }
\end{aligned}
$$

The previous line re-starts Realflex.

4. Wait about 1 minute for the system to start up and type

$$
\text { tsk | more[Return] }
$$

and verify that histdb and Wrealflex are listed.

\section{B.15. Moving the Real-Time Historical Database:}

During the summer (from 6/1 through 10/1), the following steps should be followed to backup the Kerman historical database whenever the histdata file exceeds 130,000 blocks (about 65MB).

1. cd /realflex/bin [Return]

2. start_up $+t$ [Return]

3. slay Wrealflex [Return]

4. cd/realflex/data/hist.save [Return]

5. mkdir ddmmyykmhist [Return] (ddmmyy is the current date)

6. Is [Return] (make sure the directory was created correctly)

7. cd /realflex/data/hist [Retum]

Be very careful to type the next step carefully!

8. $\mathrm{mv}$ * ../hist.save/ddmmyykmhist [Return]

9. cd/realflex/bin [Return]

10. ontty Stty0 realflex [Return] (after this command wait about 30 seconds for system to start up) 
11. tsk / more [Retum] (verify that histdb is listed in the task list)

12. Fill out an O\&M sheet and fax it to PVUSA.

\section{B.15. Restoring an Archived Real-Time Historical Database:}

Use this procedure to restore a database that has been archived in the '/realflex/data/hist.save/mmddyykmhist' directory (where mmddyy is the date the database was archived).

1. cd /realflex/bin [Return]

2. start_up +t [Return]

3. slay Wrealflex [Return]

4. cd /realflex/data/hist [Return] .

5. mkdir temp [Return] ${ }^{\circ}$

6. $\mathrm{mv}$ * temp [Return]

7. cd /realflex/data/hist.save [Return]

8. cd mmddyykmhist [Return] (where mmddyy is the date the desired database was archived)

9. $\mathrm{mv}$ */realflex/data/hist [Return]

10. cd/realflex/bin [Return]

11. ontty Stty0 realflex [Return] (wait about $\mathbf{3 0}$ seconds for system to start up)

If real-time data needs to be extracted from this database, do your hbackup command now.

If the original database needs to be re-installed, use the following procedure:

1. cd/realflex/bin [Return]

2. start_up +t [Return]

3. slay Wrealflex [Return]

4. cd /realflex/data/hist [Return]

5. $\mathrm{mv}^{*}$ *./hist.save/mmddyykmhist [Return] (where mmddyy is the same mmddyy used in step 8 above)

6. cd temp [Retum]

7. $\mathrm{mv}^{*}$.. [Return]

8. cd .. [Return]

9. rmdir temp [Return]

10. cd/realflex/bin [Return]

11. ontty \$tty0 realflex [Return]

12. tsk [Return] (to look for histdb on the list and make sure histdb is running)

\section{B.16. Directions for Re-booting System:}

1. In the top left corner of the screen is a graphic "button" with the words "MASTER MENU".

Using the mouse, position the mouse cursor over this button. Click on the mouse's right button once. 
2. A menu should appear. Position the mouse cursor over the "UTLITIES" menu item and click on the mouse's right button. Another menu should appear.

3. Choose the "EXIT REALFLEX" menu item by positioning the mouse cursor over this selection and pressing the left mouse button. There should be a single audible beep, and a message saying "Shutting Down RealFlex" should appear.

4. When this message disappears you should hear another audible beep and the screen should be blank except for a down arrow in the upper left corner of the screen. Position the cursor over this arrow and click on the left mouse button. The screen should go black, there should be some writing at the top, and there should be a command prompt (a '\$) just below the writing.

5. At this point simply push the reset button on the front panel of the computer once. The reset button is a horizontal button next to the power switch. The computer should reboot and automatically go into RealFlex.

\section{B.17. If the System is Not Responding Properly}

There are several situations where the mouse or keyboard will be operating incorrectly or not at all. The first approach is to attempt to bring down the computer as outlined in Appendix B.16. If this is unsuccessful, the next safest approach is to have the system rebooted remotely. Contact Endecon Engineering at (510) 867-3360 to perform this operation. Finally, if you are unable to have the system rebooted remotely, then you must reboot the computer via the reset button. This approach can be damaging if it is performed when the computer is writing to the hard disk. Before pressing the reset button make sure that the drive light shows no activity. The system will then reboot and bring QNX and Realflex into operation.

\section{B.18. Logging onto the R\&D Computer Via Modem}

1. Using a Hayes compatible 14.4 kbaud or slower modem (V.42bis, V.32bis, MNP 2-5 supported) configured for no parity, 8 data bits, and 1 stop bit, dial (209) 846-7958.

2. If the connection is made successfully, the following lines should be displayed:

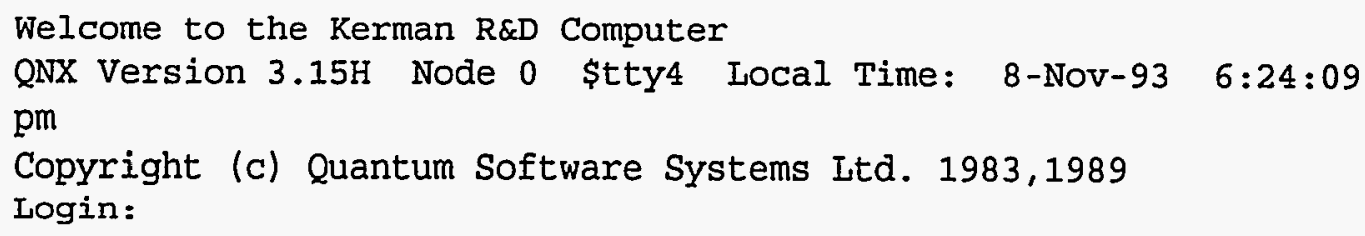

3. At the 'password:' prompt type the password.

\section{B.19. Viewing the Historical Database}

Note: You must be at the R\&D Computer at Kerman to do this.

1. Select 'Master Menu' with the left mouse button.

2. Select 'Utilities' with the left mouse button.

3. Select 'Historical Maintenance' with the right mouse button.

4. Use the cursor and page-up/down buttons to move up and down through the points list. Place the highlight line on the desired point.

5. Press F3 to edit this point.

6. Using the up/down arrows to change the values, and the Tab button to change fields, select the desired date and time to view. 
7. Press [Enter] when ready to view data. If data is entered correctly, the window should fill with the real-time data for the chosen point for the time and date specified.

8. Scroll through the data using the Page Up/Page Down buttons.

9. Press [ESC] to exit the real-time screen, and F10 to exit back to RealFlex.

\section{B.20. Changing RealFlex Passwords}

1. Click on 'Master Menu' with the left mouse button.

2. Click on 'Utilities' with the left mouse button.

3. Click on 'Change Passwords' with the right mouse button.

4. Type in the old password.

5. Follow instructions to change desired passwords.

\section{B.21. Setting the Clock}

1. From a QNX prompt type date ? [Return]

2. Following the format shown, use the date command to enter the new date and time followed by [Return].

3. That set the QNX clock, but not the internal clock. The internal clock must be set in order for the change to be permanent. To change the internal clock type rtc at +set [Return]. And example of changing the date is shown below.

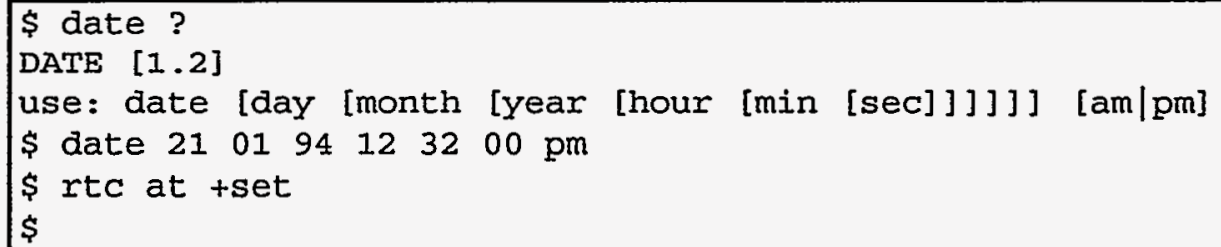


Appendix C

SCADA CONFIGURATION FILES 


\section{SCADA CONFIGURATION FILES}

\section{C.1. A003 - Harris Protocol Configuration File}

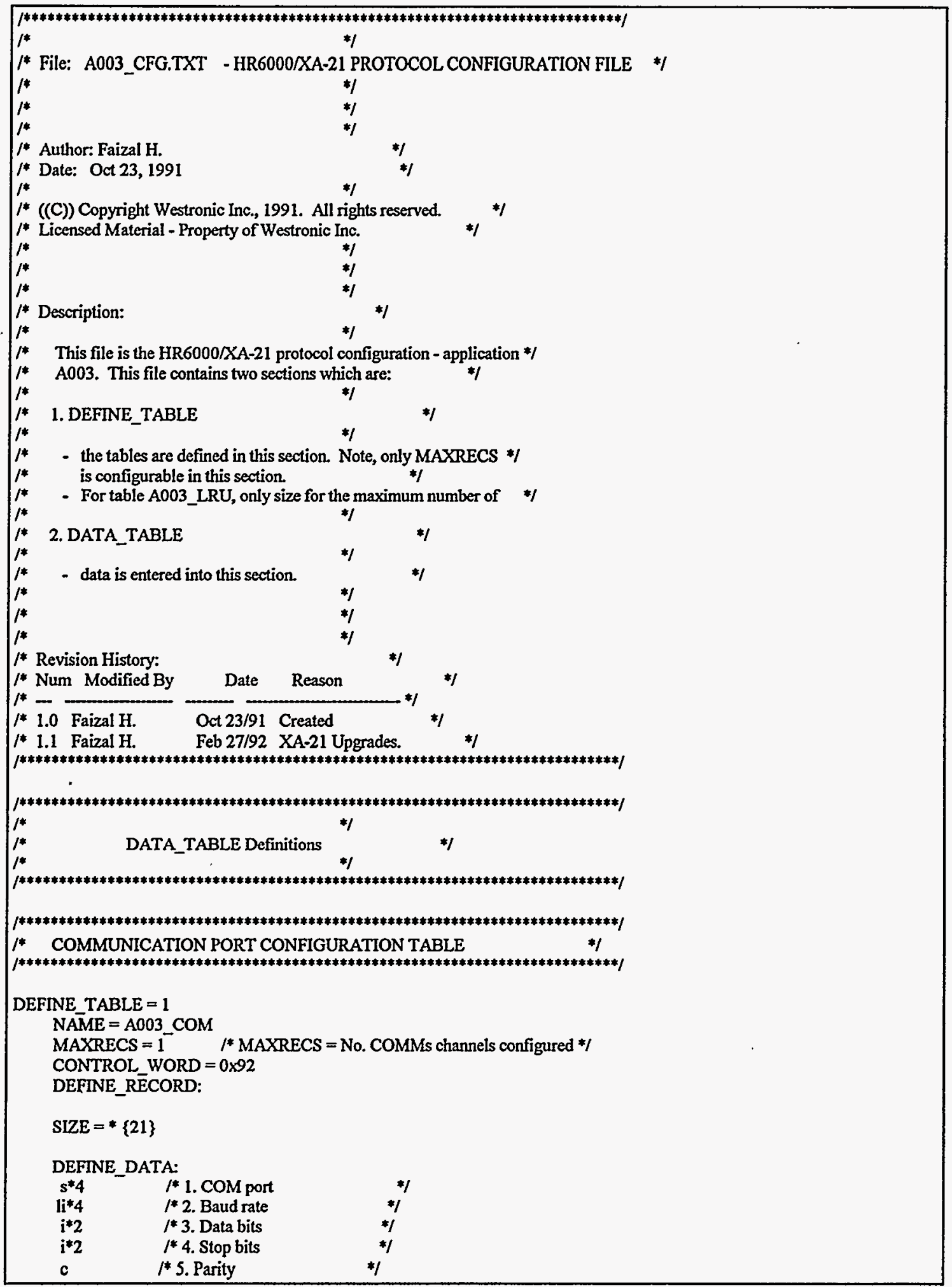




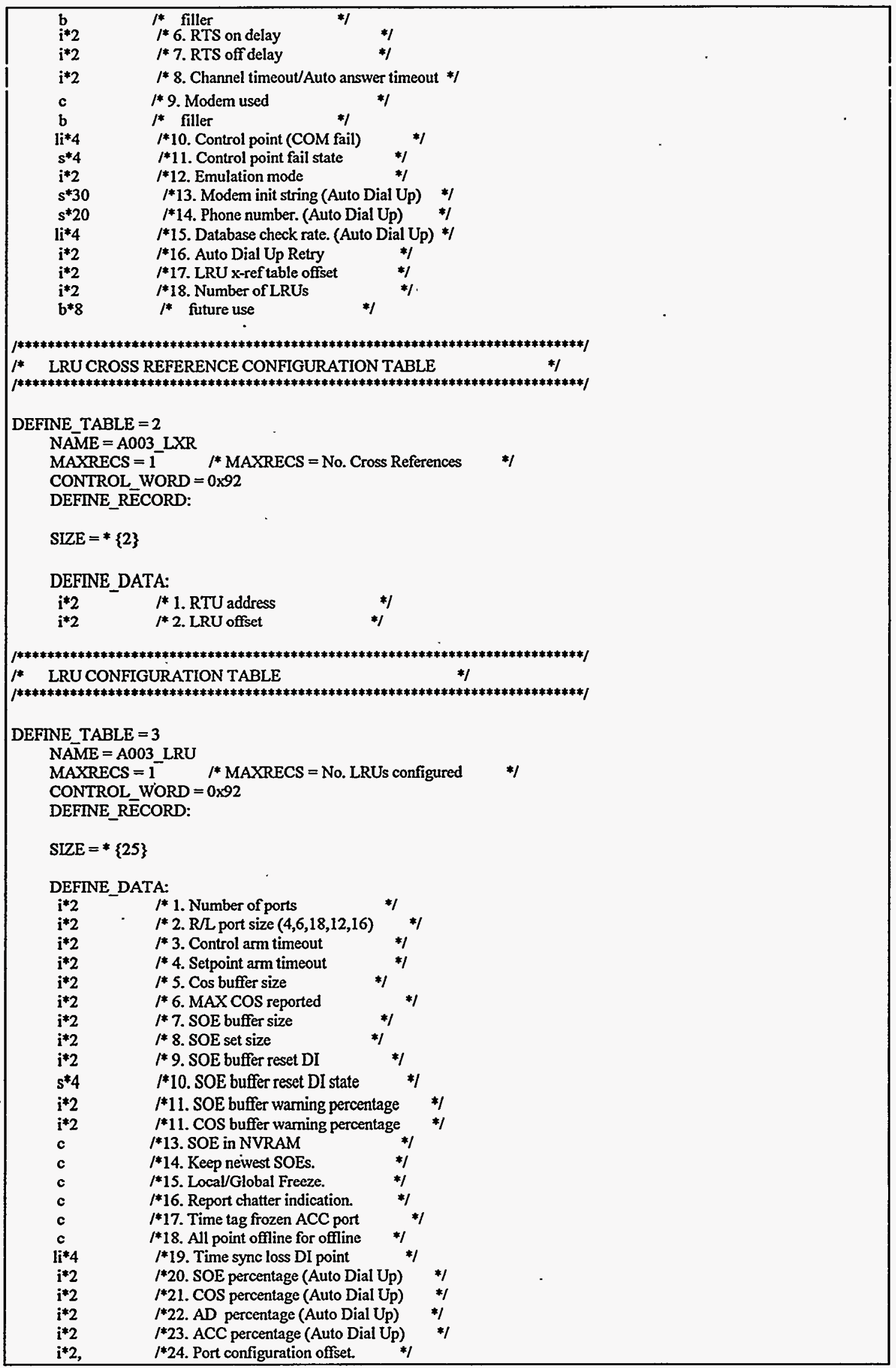




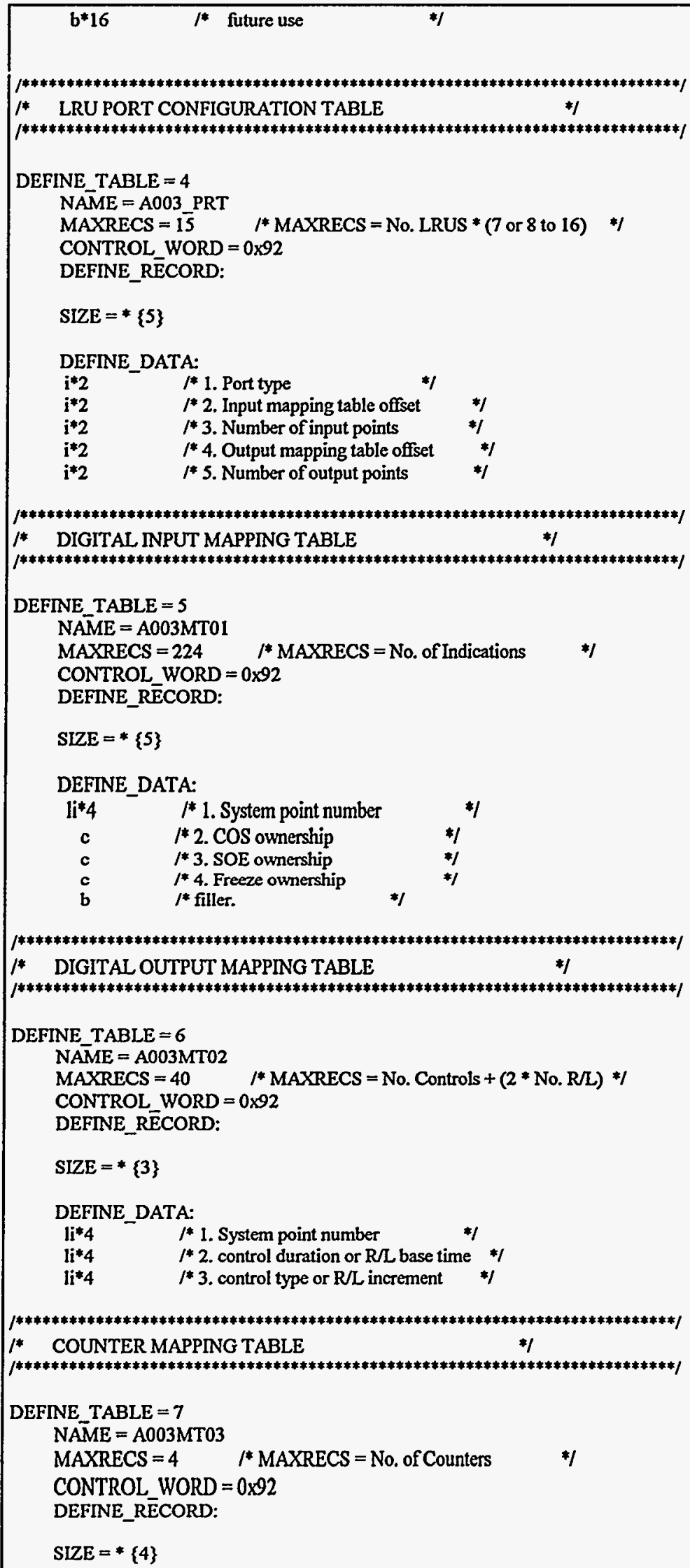




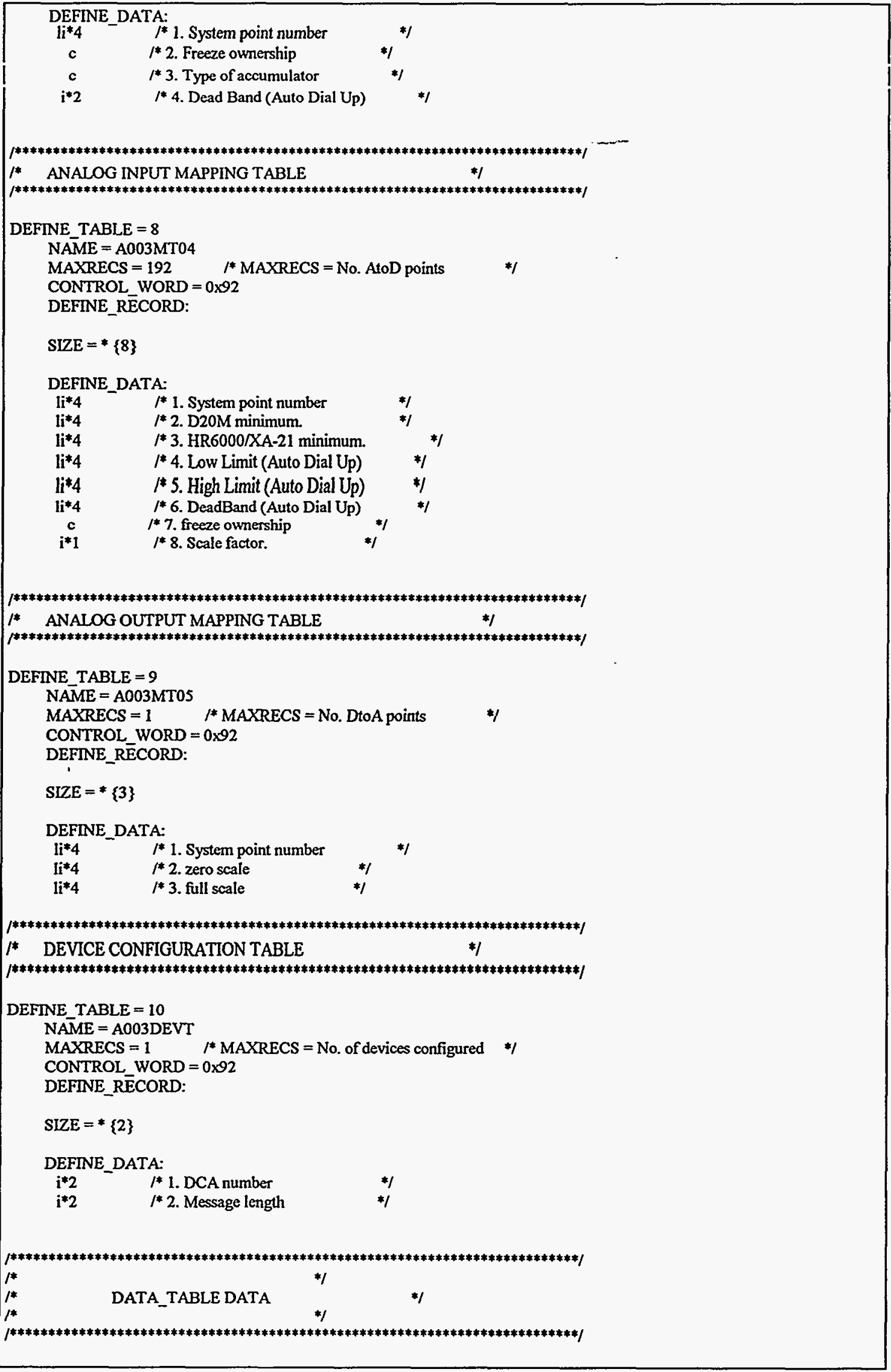




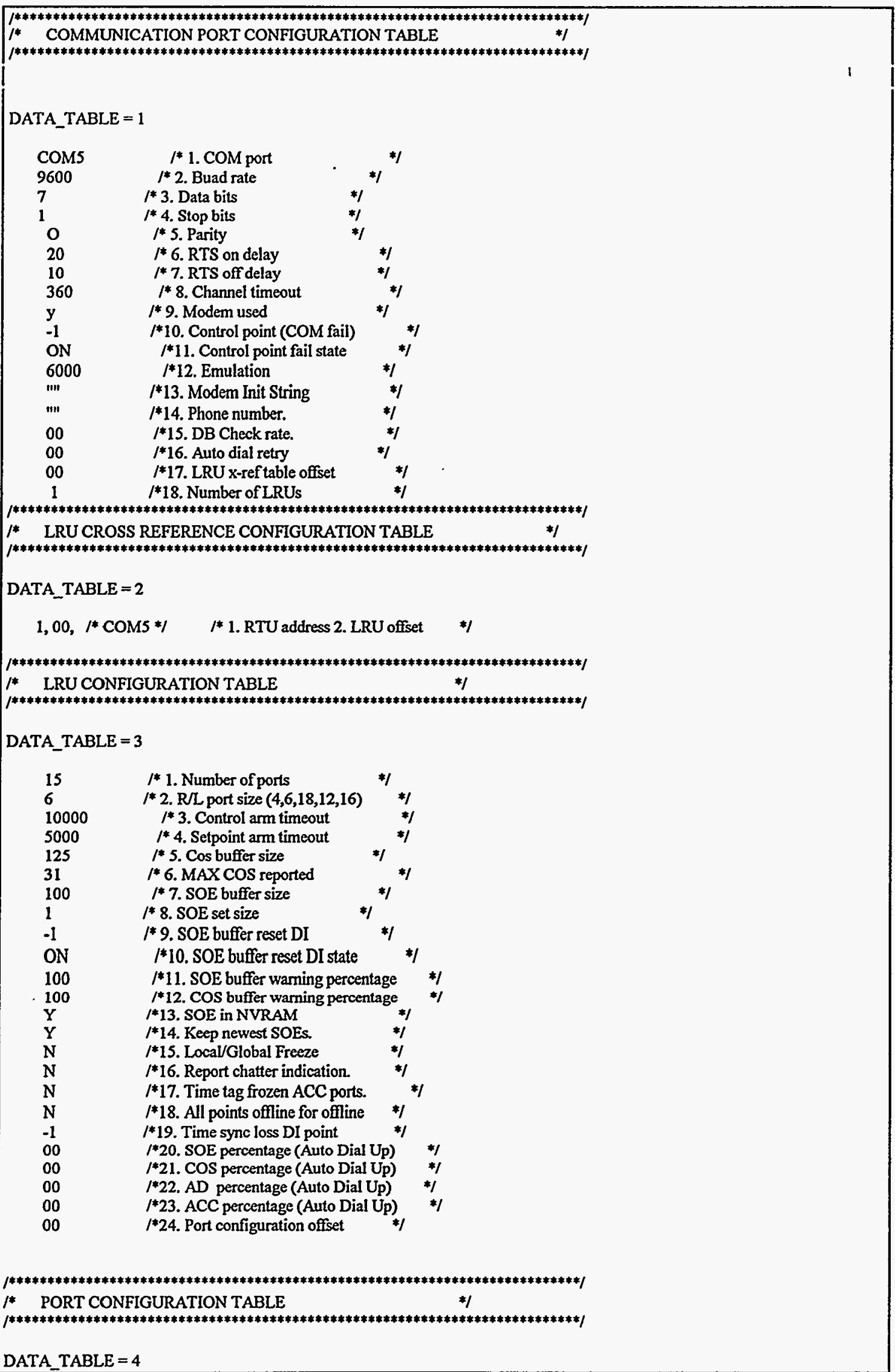




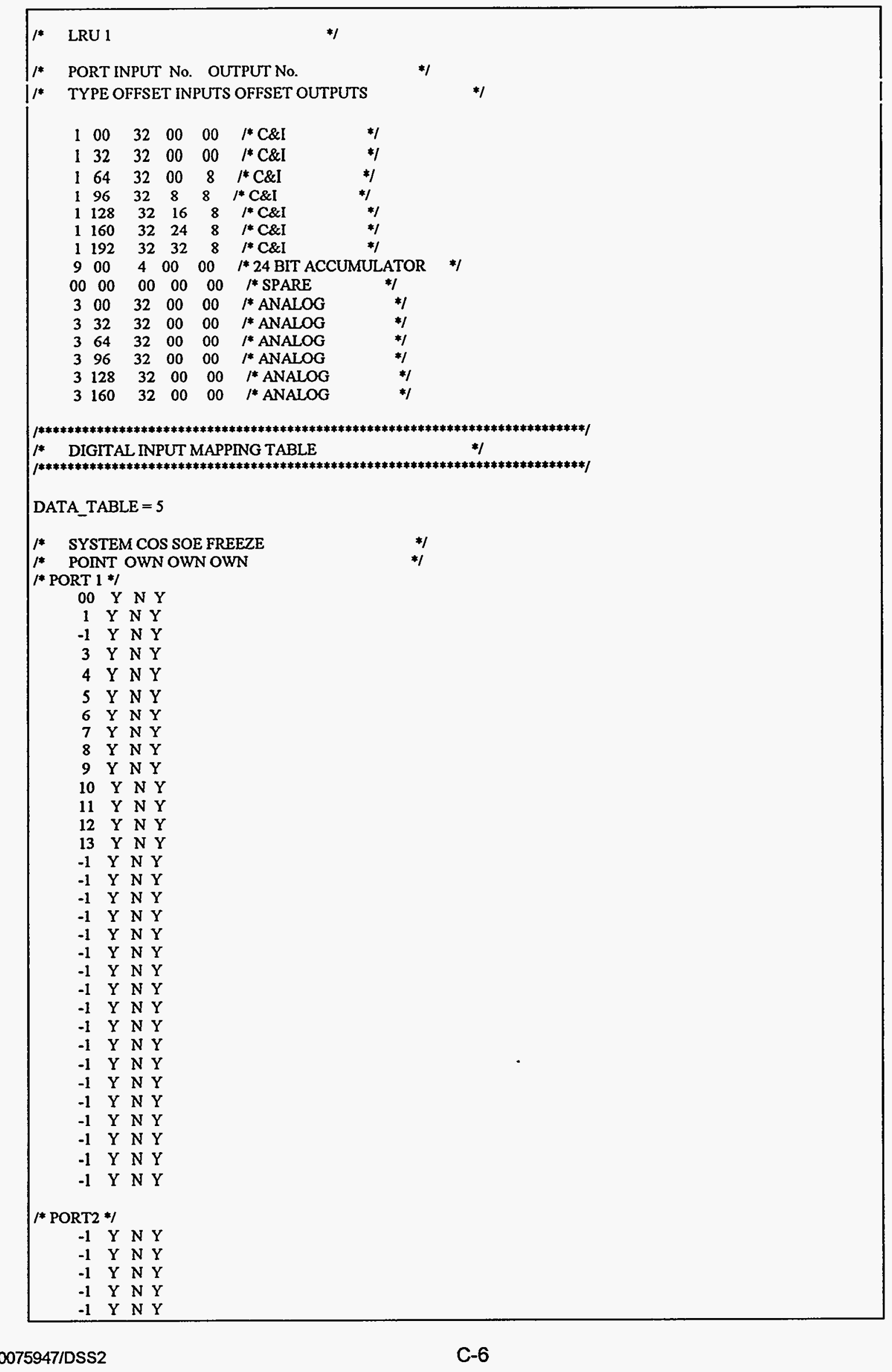




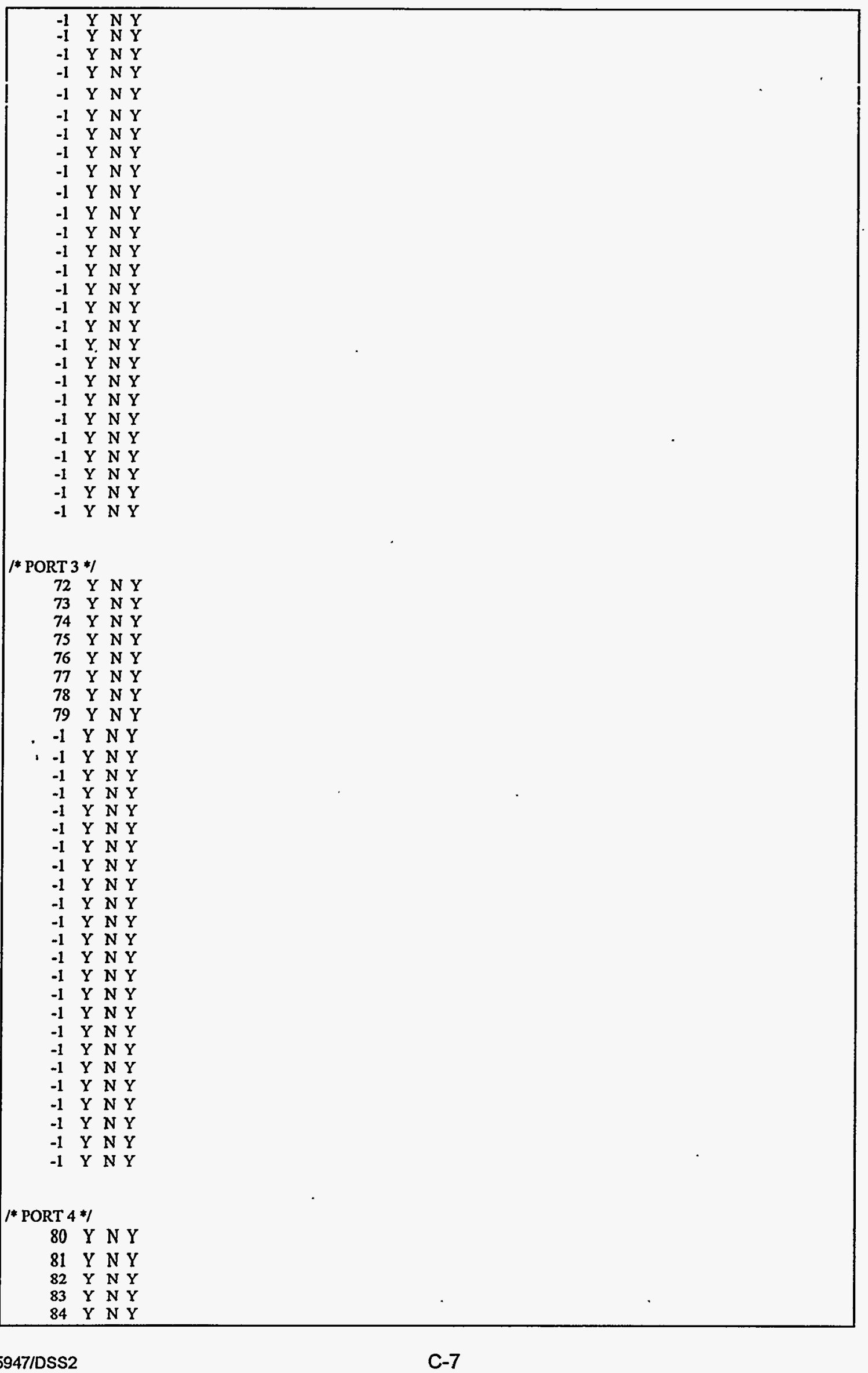




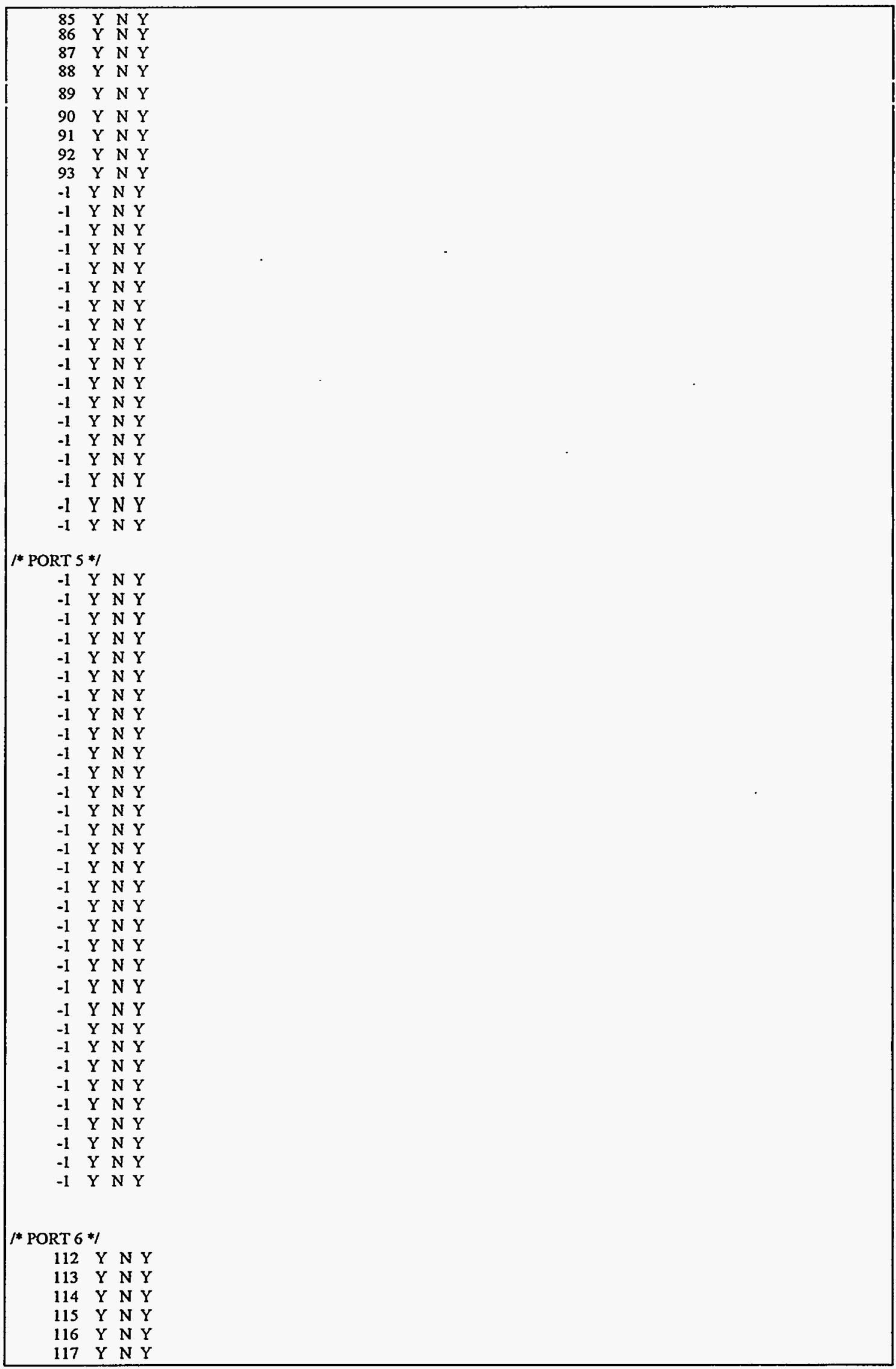




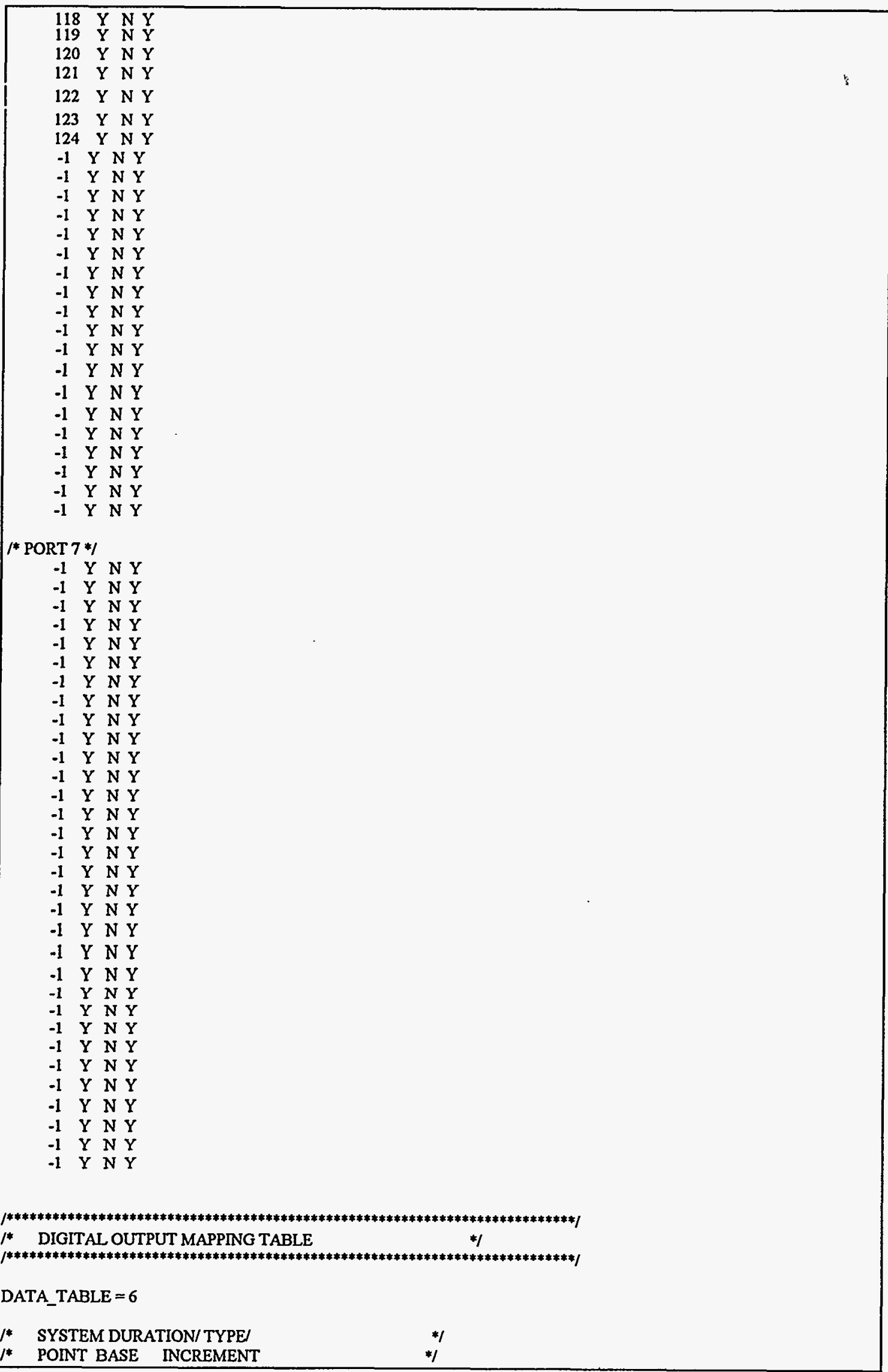




\begin{tabular}{ccc}
00 & 200 & 00 \\
1 & 200 & 00 \\
2 & 200 & 00 \\
-1 & 200 & 00 \\
-1 & 200 & 00 \\
-1 & 200 & 00 \\
-1 & 200 & 00 \\
-1 & 200 & 00 \\
& & \\
8 & 200 & 00 \\
9 & 200 & 00 \\
10 & 200 & 00 \\
11 & 200 & 00 \\
12 & 200 & 00 \\
-1 & 200 & 00 \\
-1 & 200 & 00 \\
-1 & 200 & 00 \\
& & \\
-1 & 200 & 00 \\
-1 & 200 & 00 \\
-1 & 200 & 00 \\
-1 & 200 & 00 \\
-1 & 200 & 00 \\
-1 & 200 & 00 \\
-1 & 200 & 00 \\
-1 & 200 & 00 \\
& & \\
24 & 200 & 00 \\
25 & 200 & 00 \\
26 & 200 & 00 \\
27 & 200 & 00 \\
28 & 200 & 00 \\
-1 & 200 & 00 \\
-1 & 200 & 00 \\
-1 & 200 & 00 \\
& & \\
-1 & 200 & 00 \\
-1 & 200 & 00 \\
-1 & 200 & 00 \\
-1 & 200 & 00 \\
-1 & 200 & 00 \\
-1 & 200 & 00 \\
-1 & 200 & 00 \\
-1 & 200 & 00 \\
& & \\
\hline
\end{tabular}

/******************************************************************************/ 1* COUNTER MAPPING TABLE

$*$

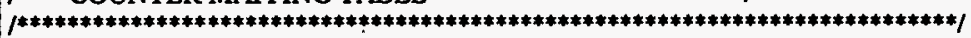

DATA_TABLE $=7$

$f^{*} \quad$ SYSTEM FREEZE TYPE DEAD

$l^{*}$ POINT OWN BAND

$$
\begin{array}{cccc}
00 & \mathrm{Y} & \mathrm{P} & 00 \\
1 & \mathrm{Y} & \mathrm{P} & 00 \\
2 & \mathrm{Y} & \mathrm{P} & 00 \\
3 & \mathrm{Y} & \mathrm{P} & 00
\end{array}
$$

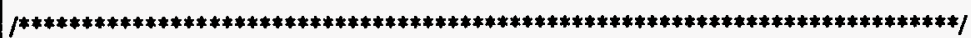

10 ANALOG INPUT MAPPING TABLE

*I

/***************************************************************************1

DATA_TABLE $=8$

i* SYSTEM D2OM HARRIS LOW HIGH DEAD FREEZE SCALE * 


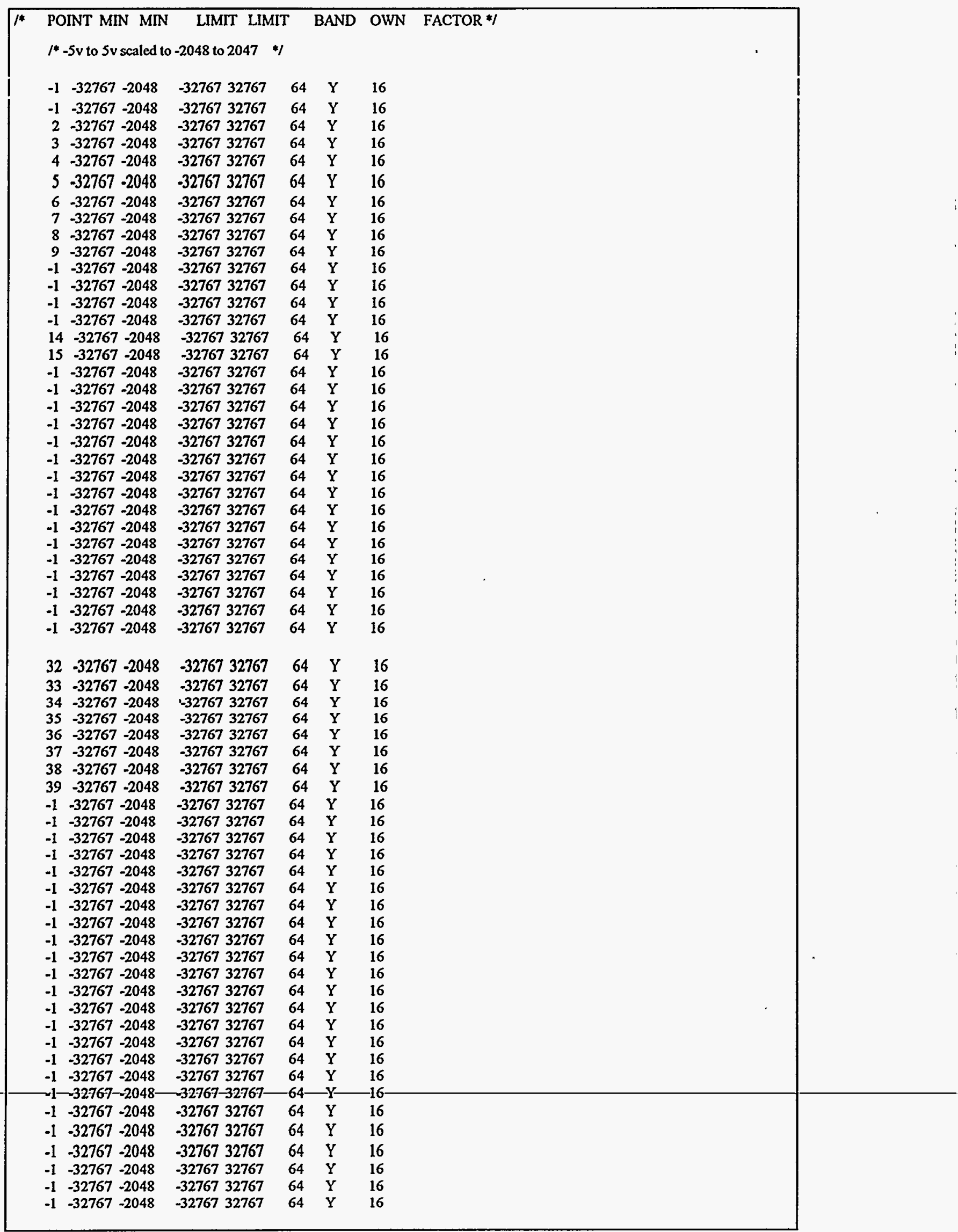




\begin{tabular}{|c|c|c|c|c|c|c|}
\hline $\begin{array}{l}48 \\
49\end{array}$ & $\begin{array}{l}-32767 \\
-32767\end{array}$ & $\begin{array}{l}-2048 \\
-2048\end{array}$ & $\begin{array}{l}-3276732767 \\
-3276732767\end{array}$ & $\begin{array}{l}64 \\
64\end{array}$ & $\begin{array}{l}Y \\
Y\end{array}$ & 16 \\
\hline 50 & -32767 & -2048 & -3276732767 & 64 & $Y$ & \\
\hline 51 & -32767 & -2048 & -3276732767 & 64 & $Y$ & \\
\hline 52 & -32767 & -2048 & -3276732767 & 64 & $\mathrm{Y}$ & \\
\hline 53 & -32767 & -2048 & -3276732767 & 64 & $\mathbf{Y}$ & \\
\hline 54 & -32767 & -2048 & -3276732767 & 64 & $\mathrm{Y}$ & \\
\hline 55 & -32767 & -2048 & -3276732767 & 64 & $\mathrm{Y}$ & \\
\hline 56 & -32767 & -2048 & -3276732767 & 64 & $\mathrm{Y}$ & \\
\hline 57 & -32767 & -2048 & -3276732767 & 64 & $\mathbf{Y}$ & \\
\hline 58 & -32767 & -2048 & -3276732767 & 64 & $\mathbf{Y}$ & \\
\hline 59 & -32767 & -2048 & -3276732767 & 64 & $\mathbf{Y}$ & \\
\hline 60 & -32767 & -2048 & -3276732767 & 64 & $\mathbf{Y}$ & \\
\hline 61 & -32767 & -2048 & -3276732767 & 64 & $\mathbf{Y}$ & \\
\hline 62 & -32767 & -2048 & -3276732767 & 64 & $\mathrm{Y}$ & \\
\hline-1 & -32767 & -2048 & -3276732767 & 64 & $\mathrm{Y}$ & 16 \\
\hline-1 & -32767 & -204 & -3276732767 & 64 & $Y$ & \\
\hline-1 & -32767 & -2048 & -3276732767 & 64 & $\mathbf{Y}$ & 16 \\
\hline-1 & -32767 & -2048 & -3276732767 & 64 & $\mathbf{Y}$ & 16 \\
\hline-1 & -32767 & -2048 & -3276732767 & 64 & $Y$ & 16 \\
\hline-1 & -32767 & -2048 & 76732767 & 64 & $Y$ & \\
\hline-1 & -32767 & -2048 & 76732767 & 64 & $\mathrm{Y}$ & 16 \\
\hline-1 & -32767 & -2048 & $-32767^{-} 32767$ & 64 & $\bar{Y}$ & 16 \\
\hline-1 & -32767 & -2048 & -3276732767 & 64 & $Y$ & 16 \\
\hline-1 & -32767 & -2048 & -3276732767 & 64 & $\mathrm{Y}$ & 16 \\
\hline-1 & -32767 & -2048 & -3276732767 & 64 & $\mathrm{Y}$ & 16 \\
\hline-1 & -32767 & -2048 & -3276732767 & 64 & $\mathbf{Y}$ & 16 \\
\hline-1 & -32767 & -2048 & -3276732767 & 64 & $\mathbf{Y}$ & 16 \\
\hline-1 & -32767 & -2048 & -3276732767 & 64 & $\mathbf{Y}$ & 16 \\
\hline-1 & -32767 & -2048 & -3276732767 & 64 & $Y$ & 16 \\
\hline-1 & -32767 & -2048 & -3276732767 & 64 & $\mathrm{Y}$ & 16 \\
\hline-1 & -32767 & -2048 & -3276732767 & 64 & $\mathbf{Y}$ & 16 \\
\hline 64 & -32767 & -2048 & -3276732767 & 64 & $\mathrm{Y}$ & 1 \\
\hline 65 & -32767 & -2048 & -3276732767 & 64 & $\mathbf{Y}$ & \\
\hline 66 & -32767 & -2048 & -3276732767 & 64 & $\mathbf{Y}$ & \\
\hline 67 & -32767 & -2048 & -3276732767 & 64 & $\mathbf{Y}$ & 10 \\
\hline 68 & & -2048 & -3276732767 & 64 & $\mathbf{Y}$ & 1 \\
\hline-1 & -32767 & -2048 & -3276732767 & 64 & $\mathbf{Y}$ & 16 \\
\hline-1 & -32767 & -2048 & -3276732767 & 64 & $\mathbf{Y}$ & 16 \\
\hline-1 & -32767 & -2048 & -3276732767 & 64 & $\mathbf{Y}$ & 16 \\
\hline 72 & & -2048 & $-32767 \quad 32767$ & -64 & $Y$ & -1 \\
\hline 73 & -32767 & -2048 & -3276732767 & 64 & $\mathbf{Y}$ & \\
\hline 74 & -32767 & -2048 & -3276732767 & 64 & $Y$ & I6 \\
\hline 75 & -32767 & -2048 & -3276732767 & 64 & $\mathbf{Y}$ & 16 \\
\hline 76 & -32767 & -2048 & -3276732767 & 64 & $\mathbf{Y}$ & 16 \\
\hline-1 & -32767 & -2048 & -3276732767 & 64 & $\mathbf{Y}$ & 16 \\
\hline-1 & -32767 & -2048 & -3276732767 & 64 & $\mathbf{Y}$ & 16 \\
\hline-1 & -32767 & -2048 & -3276732767 & 64 & $\mathbf{Y}$ & 16 \\
\hline-1 & -32767 & -2048 & -3276732767 & 64 & $\mathbf{Y}$ & 16 \\
\hline-1 & -32767 & -2048 & -3276732767 & 64 & $\mathbf{Y}$ & 16 \\
\hline-1 & -32767 & -2048 & -3276732767 & 64 & $\mathrm{Y}$ & 16 \\
\hline-1 & -32767 & -2048 & -32767327 & 64 & $Y$ & 16 \\
\hline-1 & -32767 & -2048 & -3276732767 & 64 & $\mathbf{Y}$ & 16 \\
\hline-1 & -32767 & -2048 & -3276732767 & 64 & $\mathrm{Y}$ & 16 \\
\hline-1 & -32767 & -2048 & -3276732767 & 64 & $\mathbf{Y}$ & 16 \\
\hline-1 & -32767 & -2048 & -327673276 & 64 & $\mathrm{Y}$ & 16 \\
\hline-1 & -32767 & -2048 & -3276732767 & 64 & $Y$ & 16 \\
\hline-1 & -32767 & -2048 & -3276732767 & 64 & $\mathbf{Y}$ & 16 \\
\hline-1 & -32767 & -2048 & -3276732767 & 64 & $\mathrm{Y}$ & 16 \\
\hline-1 & & -2048 & -3276732767 & 64 & $\mathbf{Y}$ & 16 \\
\hline-1 & -32767 & -2048 & -3276732767 & 64 & $Y$ & 16 \\
\hline-1 & & -2048 & -3276732767 & 64 & $\mathrm{Y}$ & 16 \\
\hline-1 & -32767 & -2048 & -3276732767 & 64 & $\mathrm{Y}$ & 16 \\
\hline-1 & -32767 & -2048 & -3276732767 & 64 & $\mathbf{Y}$ & 16 \\
\hline 80 & & -2048 & -3276732767 & 64 & $\mathrm{Y}$ & 16 \\
\hline 81 & -32767 & -2048 & -3276732767 & 64 & $\mathbf{Y}$ & 10 \\
\hline 82 & -32767 & -2048 & -3276732767 & 64 & $\mathrm{Y}$ & 16 \\
\hline 83 & -32767 & -2048 & -3276732767 & 64 & $\mathbf{Y}$ & 16 \\
\hline
\end{tabular}




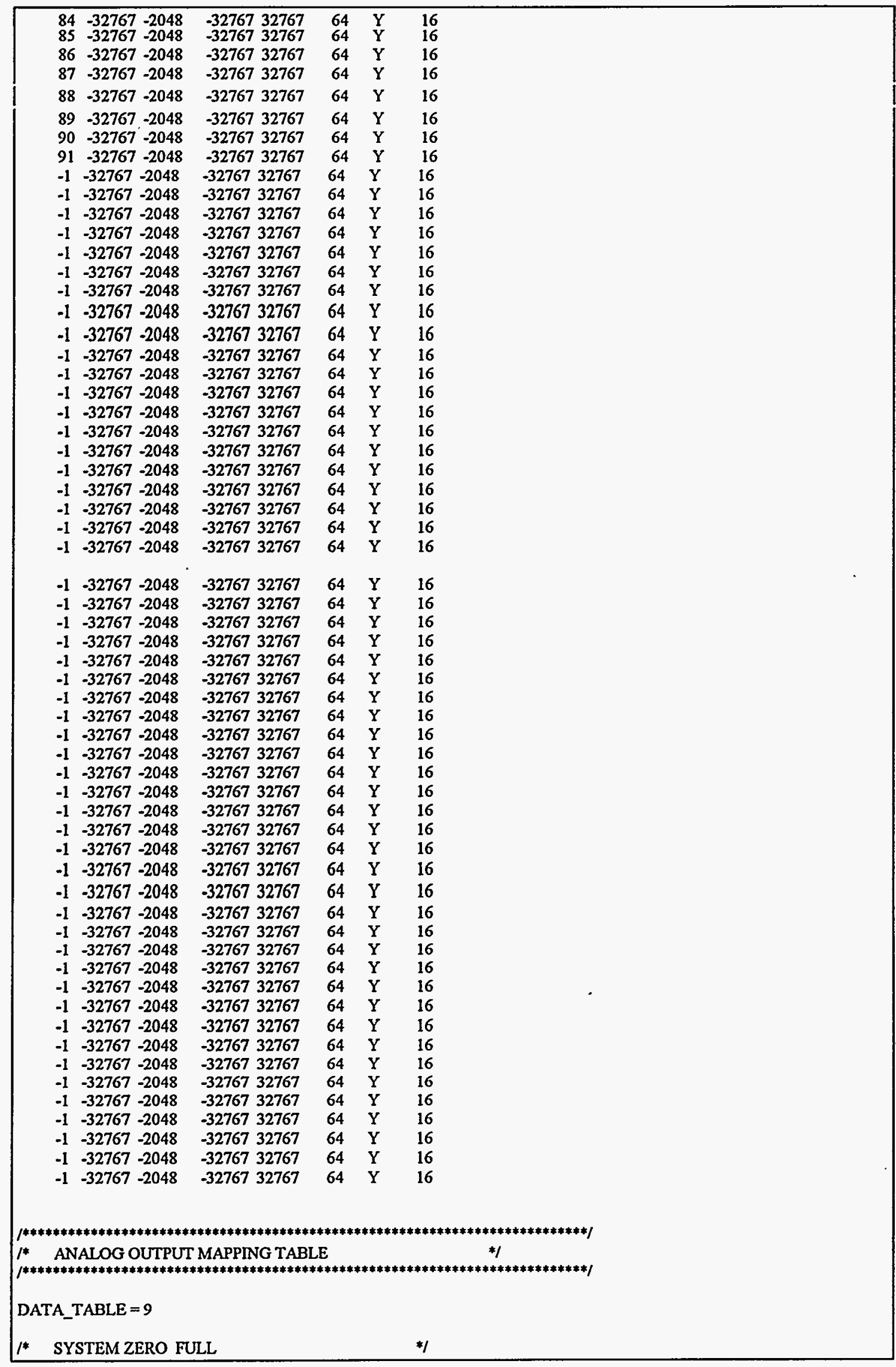


/* POINT SCALE SCALE

$*$

-1 $6553532767 \quad / * 4$ to $20 \mathrm{~mA}$ range (20mA adaptors)

*1

$|/ * * * * * * * * * * * * * * * * * * * * * * * * * * * * * * * * * * * * * * * * * * * * * * * * * * * * * * * * * * * * * * * * * * * * * * * * * *|$

/* DEVICE CONFIGURATION TABLE */

$/ * * * * * * * * * * * * * * * * * * * * * * * * * * * * * * * * * * * * * * * * * * * * * * * * * * * * * * * * * * * * * * * * * * * * * * * * * * * * * * \mid$

DATA_TABLE $=10$

/* DCA MESSAGE

/* NUMBER LENGTH

$*$

$*$

$1, \quad 256$

$*$ 


\section{C.2. A009 - PG\&E Protocol Configuration File}

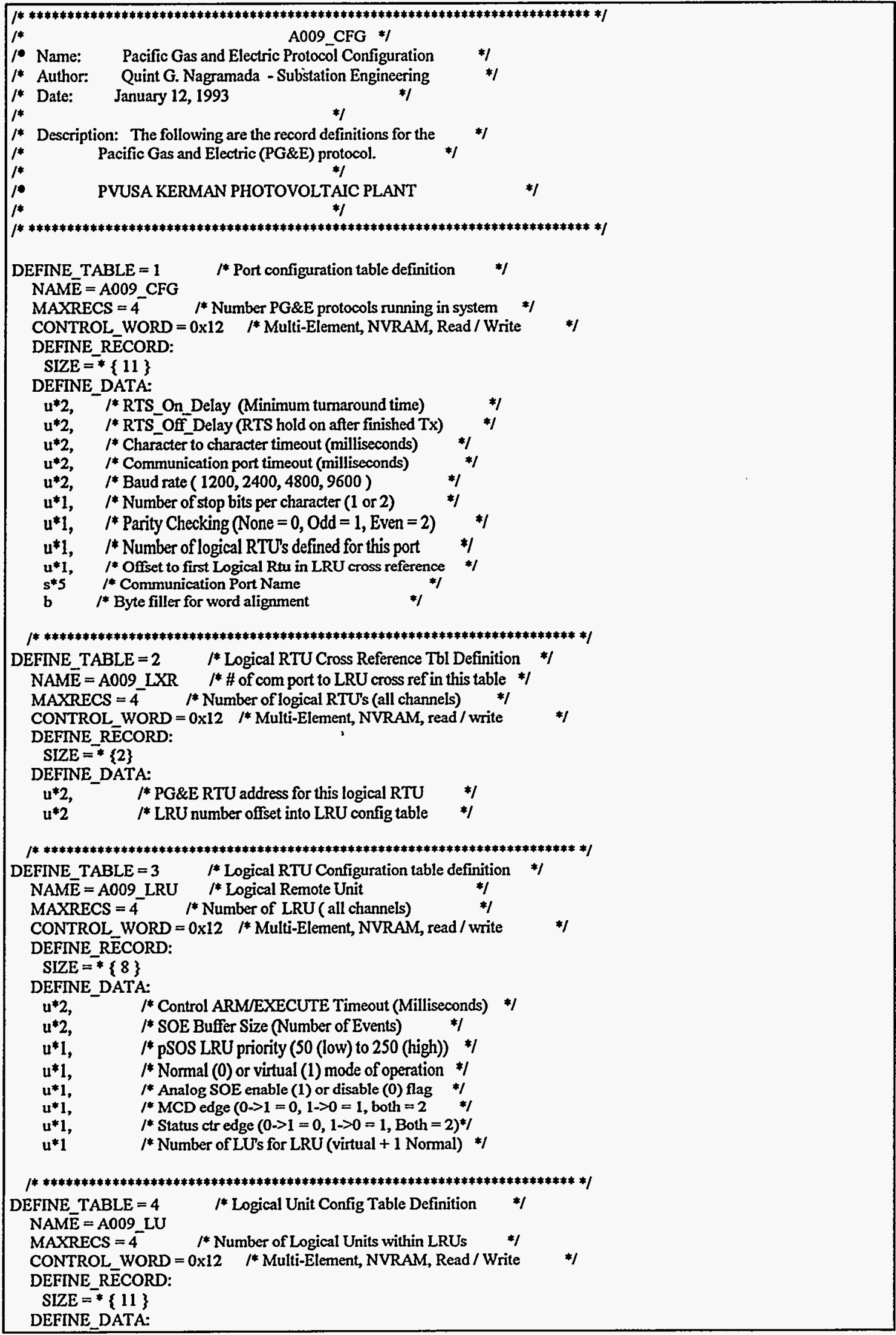




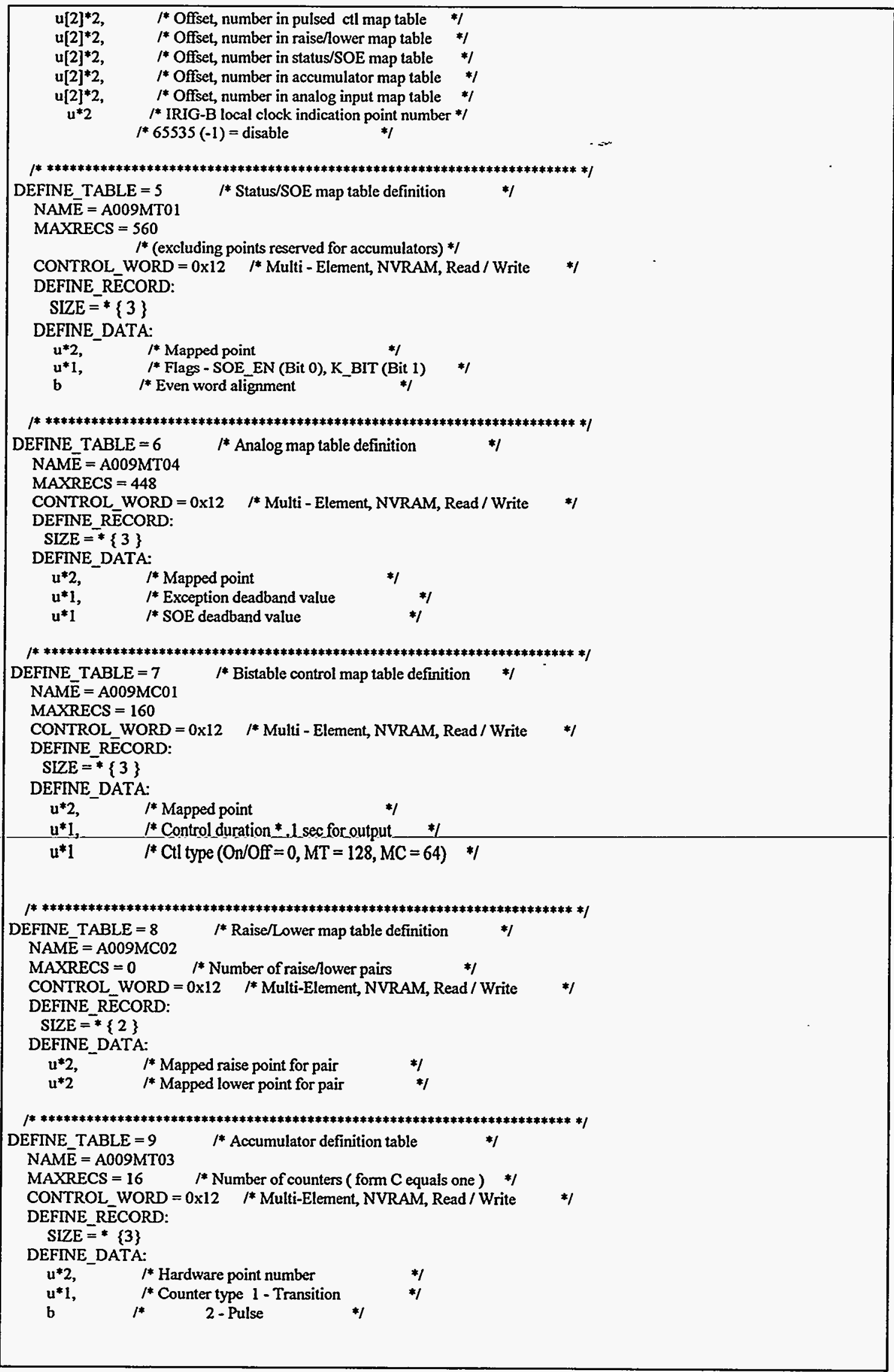




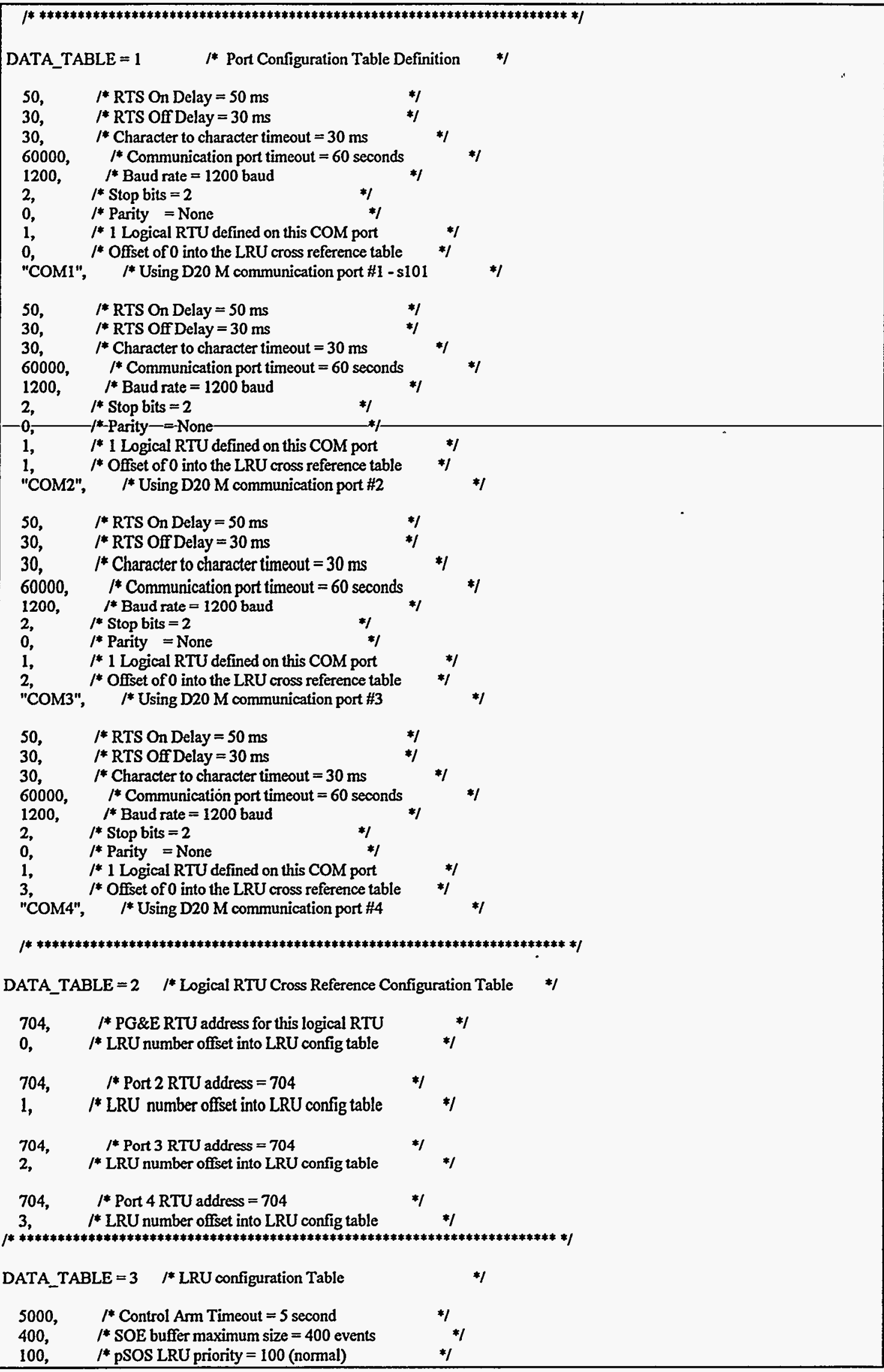




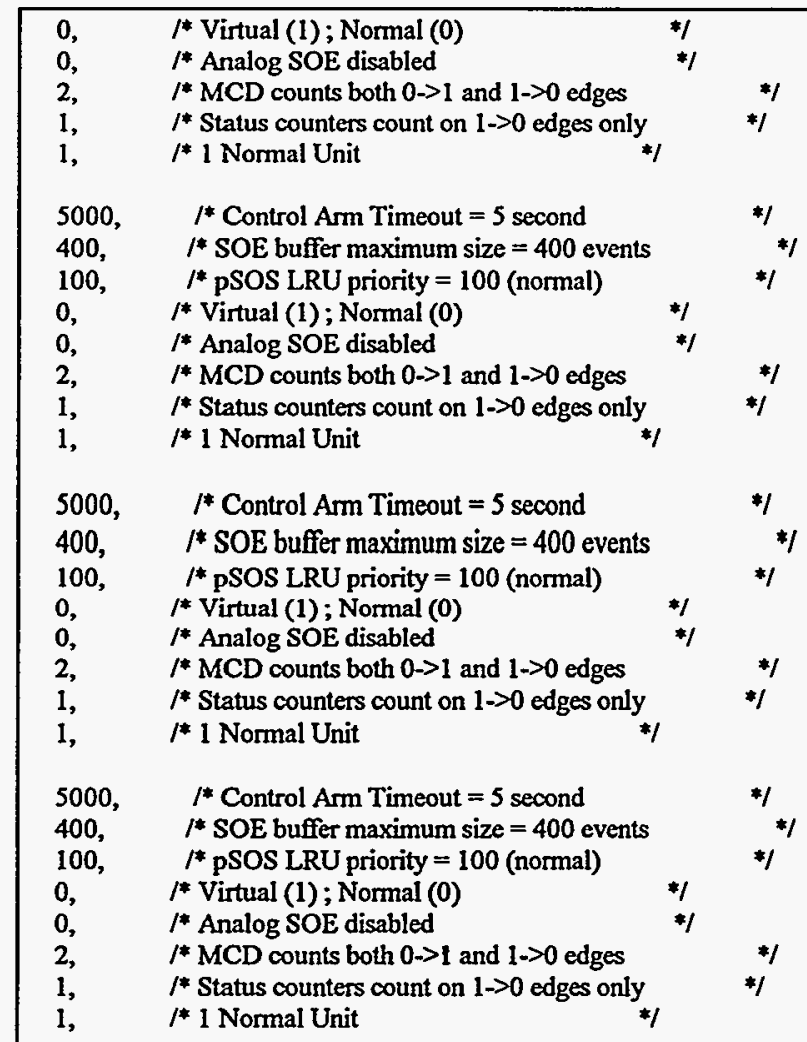

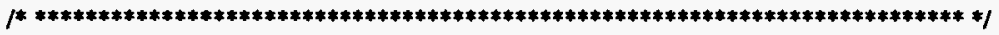

DATA_TABLE $=4 \quad / *$ L.U. Configuration Table Definition * $\quad$ \%

1* Port 1 LU's config

$*$

$1 *$ Offset, number in normal mode ctl. output map tbl 1* Offset, number in normal mode raise/lower map tbl 1* Offset, number in normal mode status/SOE map tbl $1 *$ Offset, number in normal mode accumulator map tbl $l^{*}$ Offset, number in normal mode analog input map tbl

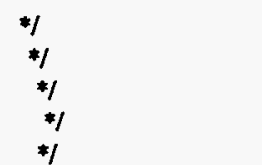

00,40 ,

00,00 ,

00,140 ,

00,4 ,

00,112 ,

$151, l^{*}$ IRIG-B local clock indication point number $\quad$ */

/* Port 2 LU's config

$*$

$l^{*}$ Offset, number in normal mode ctl. output map tbl $1 *$ Offset, number in normal mode raise/lower map tbl 1* Offset, number in normal mode status/SOE map tbl /* Offset, number in normal mode accumulator map tbl

1* Offset, number in normal mode analog input map tbl

$* 1$
$* 1$
$* 1$
$* I$

40,40 ,

00,00 ,

140,140

I* Normal mode unit, */

4,4 ,

112,112

$151,1^{*}$ IRIG-B local clock indication point number 
$1 *$ Offset, number in normal mode ctl. output map tbl $1^{*}$ Offset, number in normal mode raise/lower map tbl 1* Offset, number in normal mode status/SOE map tbl $1^{*}$ Offset, number in normal mode accumulator map tbl /* Offset, number in normal mode analog input map tbl

80,40 ,

00,00 ,

280, 140, $\quad / *$ Normal mode unit, */

8,4 ,

224,112 ,

151, /* IRIG-B local clock indication point number

$*$

/* Port 4 LU's config

$*$ I

$1^{*}$ Offset, number in normal mode ctl. output map tbl $I^{*}$ Offset, number in normal mode raise/lower map $|b|$ $1^{*}$ Offset, number in normal mode status/SOE map tbl $I^{*}$ Offset, number in normal mode accumulator map tbl I* Offset, number in normal mode analog input map tbl

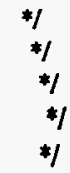

120,40 ,

00,00 ,

420,140 ,

I* Normal mode unit, */

12,4 ,

336,112 ,

$151, / *$ IRIG-B local clock indication point number

$*$

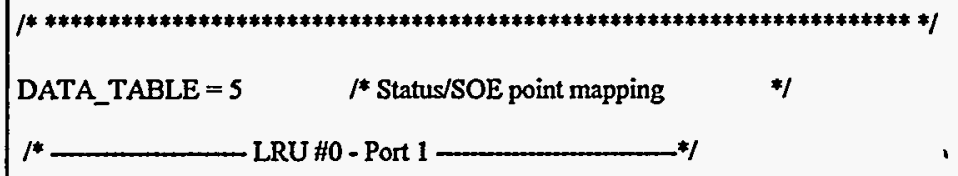

$0,3,1,3,2,3,3,3,4,3,5,3,6,3,7,3,8,3,9,3$,

$10,3,11,3,12,3,13,3,14,3,15,3,16,3,17,3,18,3,19,3$,

$20,3,21,3,22,3,23,3,24,3,25,3,26,3,27,3,28,3,29,3$,

$30,3,31,3,22,3,33,3,34,3,35,3,36,3,37,3,38,3,39,3$,

$40,3,41,3,42,3,43,3,44,3,45,3,46,3,47,3,48,3,49,3$,

$50,3,51,3,52,3,53,3,54,3,55,3,56,3,57,3,58,3,59,3$,

$60,3,61,3,62,3,63,3,6 \quad 68,3,69,3$,

$70,3,71,3,72,3,73,3,74,3,75,3,76,3,77,3,78,3,79,3$,

$80,3,81,3,82,3,83,3,84,3,85,3,86,3,87,3,88,3,89,3$,

$90,3,91,3,92,3,93,3,94,3,95,3,96,3,97,3,98,3,99,3$,

$100,3,101,3,102,3,103,3,104,3,105,3,106,3,107,3,108,3,109,3$,

$110,3,111,3,112,3,113,3,114,3,115,3,116,3,117,3,118,3,119,3$,

$120,3,121,3,122,3,123,3,124,3,125,3,126,3,127,3,128,3,129,3$,

$130,3,131,3,132,3,133,3,134,3,135,3,136,3,137,3,138,3,139,3$,

$140,3,141,3,142,3,143,3$,

$1 *$

LRU\#1 - Port 2

$0,3,1,3,2,3,3,3,4,3,5,3,6,3,7,3,8,3,9,3$,

$10,3,11,3,12,3,13,3,14,3,15,3,16,3,17,3,18,3,19,3$,

$20,3,21,3,22,3,23,3,24,3,25,3,26,3,27,3,28,3,29,3$,

$30,3,31,3,22,3,33,3,34,3,35,3,36,3,37,3,38,3,39,3$,

$40,3,41,3,42,3,43,3,44,3,45,3,46,3,47,3,48,3,49,3$,

$50,3,51,3,52,3,53,3,54,3,55,3,56,3,57,3,58,3,59,3$,

$60,3,61,3,62,3,63,3$,

$68,3,69,3$

$70,3,71,3,72,3,73,3,74,3,75,3,76,3,77,3,78,3,79,3$,

$80,3,81,3,82,3,83,3,84,3,85,3,86,3,87,3,88,3,89,3$,

$90,3,91,3,92,3,93,3,94,3,95,3,96,3,97,3,98,3,99,3$, 


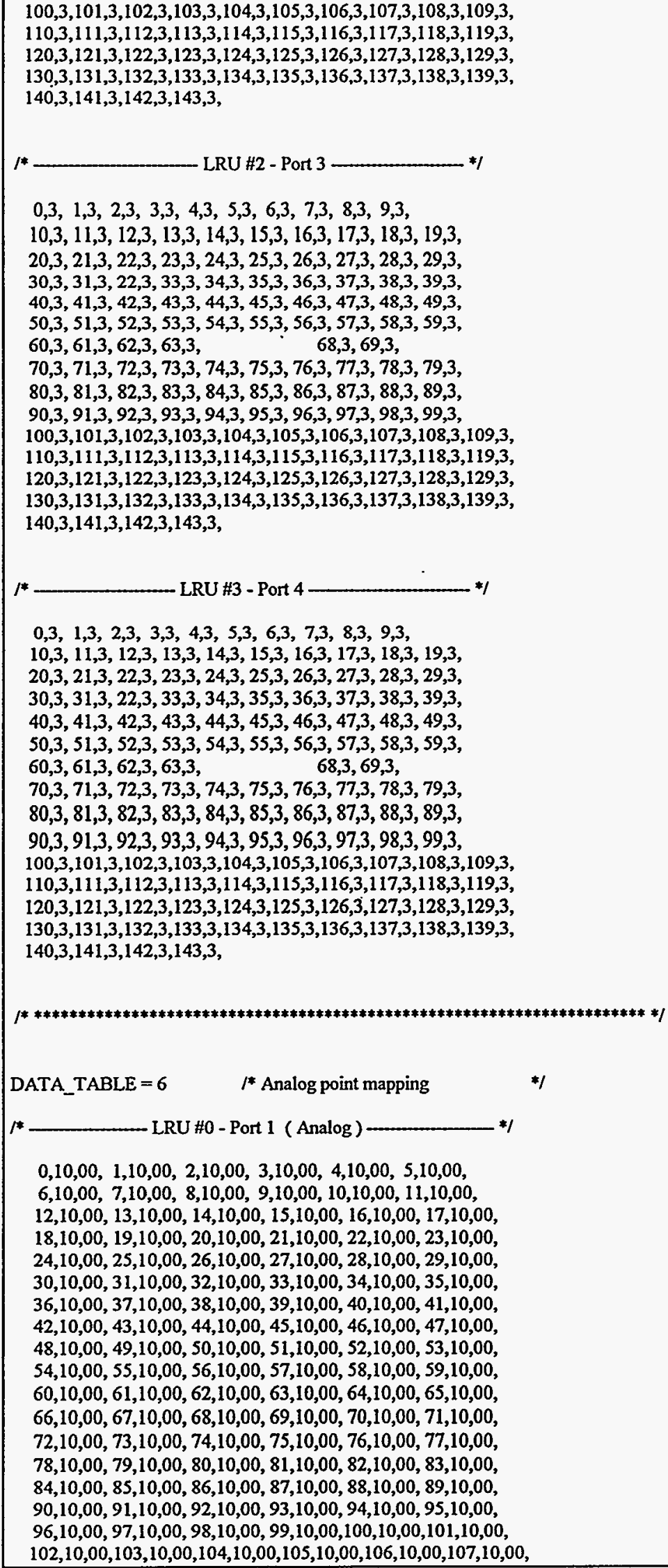

$0,3,1,3,2,3,3,3,4,3,5,3,6,3,7,3,8,3,9,3$, $10,3,11,3,12,3,13,3,14,3,15,3,16,3,17,3,18,3,19,3$, $20,3,21,3,22,3,23,3,24,3,25,3,26,3,27,3,28,3,29,3$, $30,3,31,3,22,3,33,3,34,3,35,3,36,3,37,3,38,3,39,3$, $40,3,41,3,42,3,43,3,44,3,45,3,46,3,47,3,48,3,49,3$, $50,3,51,3,52,3,53,3,54,3,55,3,56,3,57,3,58,3,59,3$, $60,3,61,3,62,3,63,3, \quad 68,3,69,3$ $70,3,71,3,72,3,73,3,74,3,75,3,76,3,77,3,78,3,79,3$, $80,3,81,3,82,3,83,3,84,3,85,3,86,3,87,3,88,3,89,3$, $90,3,91,3,92,3,93,3,94,3,95,3,96,3,97,3,98,3,99,3$, $100,3,101,3,102,3,103,3,104,3,105,3,106,3,107,3,108,3,109,3$, $110,3,111,3,112,3,113,3,114,3,115,3,116,3,117,3,118,3,119,3$ $120,3,121,3,122,3,123,3,124,3,125,3,126,3,127,3,128,3,129,3$, $130,3,131,3,132,3,133,3,134,3,135,3,136,3,137,3,138,3,139,3$, $140,3,141,3,142,3,143,3$,

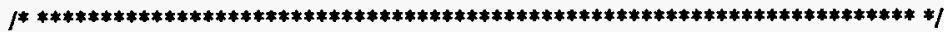
DATA_TABLE $=6 \quad$ I* Analog point mapping *I $f^{*} \longrightarrow$ LRU \#0 - Port 1 (Analog)

$0,10,00,1,10,00,2,10,00,3,10,00,4,10,00,5,10,00$, $6,10,00,7,10,00,8,10,00,9,10,00,10,10,00,11,10,00$, $12,10,00,13,10,00,14,10,00,15,10,00,16,10,00,17,10,00$, $18,10,00,19,10,00,20,10,00,21,10,00,22,10,00,23,10,00$, $24,10,00,25,10,00,26,10,00,27,10,00,28,10,00,29,10,00$, $30,10,00,31,10,00,32,10,00,33,10,00,34,10,00,35,10,00$, $36,10,00,37,10,00,38,10,00,39,10,00,40,10,00,41,10,00$, $42,10,00,43,10,00,44,10,00,45,10,00,46,10,00,47,10,00$, $48,10,00,49,10,00,50,10,00,51,10,00,52,10,00,53,10,00$, $54,10,00,55,10,00,56,10,00,57,10,00,58,10,00,59,10,00$, $60,10,00,61,10,00,62,10,00,63,10,00,64,10,00,65,10,00$, $66,10,00,67,10,00,68,10,00,69,10,00,70,10,00,71,10,00$, $72,10,00,73,10,00,74,10,00,75,10,00,76,10,00,77,10,00$, $78,10,00,79,10,00,80,10,00,81,10,00,82,10,00,83,10,00$, $84,10,00,85,10,00,86,10,00,87,10,00,88,10,00,89,10,00$, $90,10,00,91,10,00,92,10,00,93,10,00,94,10,00,95,10,00$, $96,10,00,97,10,00,98,10,00,99,10,00,100,10,00,101,10,00$, $102,10,00,103,10,00,104,10,00,105,10,00,106,10,00,107,10,00$, 
$108,10,00,109,10,00,110,10,00,111,10,00$

$I^{*} \longrightarrow$ LRU \#1 - Port 2 (Analog) ___ *I

$0,10,00,1,10,00,2,10,00,3,10,00,4,10,00,5,10,00$, $6,10,00,7,10,00,8,10,00,9,10,00,10,10,00,11,10,00$, $12,10,00,13,10,00,14,10,00,15,10,00,16,10,00,17,10,00$, $18,10,00,19,10,00,20,10,00,21,10,00,22,10,00,23,10,00$, $24,10,00,25,10,00,26,10,00,27,10,00,28,10,00,29,10,00$, $30,10,00,31,10,00,32,10,00,33,10,00,34,10,00,35,10,00$, $36,10,00,37,10,00,38,10,00,39,10,00,40,10,00,41,10,00$, $42,10,00,43,10,00,44,10,00,45,10,00,46,10,00,47,10,00$, $48,10,00,49,10,00,50,10,00,51,10,00,52,10,00,53,10,00$, $54,10,00,55,10,00,56,10,00,57,10,00,58,10,00,59,10,00$, $60,10,00,61,10,00,62,10,00,63,10,00,64,10,00,65,10,00$, $66,10,00,67,10,00,68,10,00,69,10,00,70,10,00,71,10,00$, $72,10,00,73,10,00,74,10,00,75,10,00,76,10,00,77,10,00$, $78,10,00,79,10,00,80,10,00,81,10,00,82,10,00,83,10,00$, $84,10,00,85,10,00,86,10,00,87,10,00,88,10,00,89,10,00$, $90,10,00,91,10,00,92,10,00,93,10,00,94,10,00,95,10,00$, $96,10,00,97,10,00,98,10,00,99,10,00,100,10,00,101,10,00$, $102,10,00,103,10,00,104,10,00,105,10,00,106,10,00,107,10,00$, $108,10,00,109,10,00,110,10,00,111,10,00$,

$1 *$ LRU \#2 - Port 3 (Analog) —_

$0,10,00,1,10,00,2,10,00,3,10,00,4,10,00,5,10,00$, $6,10,00,7,10,00,8,10,00,9,10,00,10,10,00,11,10,00$, $12,10,00,13,10,00,14,10,00,15,10,00,16,10,00,17,10,00$, $18,10,00,19,10,00,20,10,00,21,10,00,22,10,00,23,10,00$, $24,10,00,25,10,00,26,10,00,27,10,00,28,10,00,29,10,00$, $30,10,00,31,10,00,32,10,00,33,10,00,34,10,00,35,10,00$, $36,10,00,37,10,00,38,10,00,39,10,00,40,10,00,41,10,00$, $42,10,00,43,10,00,44,10,00,45,10,00,46,10,00,47,10,00$, $48,10,00,49,10,00,50,10,00,51,10,00,52,10,00,53,10,00$, $54,10,00,55,10,00,56,10,00,57,10,00,58,10,00,59,10,00$, $60,10,00,61,10,00,62,10,00,63,10,00,64,10,00,65,10,00$, $66,10,00,67,10,00,68,10,00,69,10,00,70,10,00,71,10,00$, $72,10,00,73,10,00,74,10,00,75,10,00,76,10,00,77,10,00$, $78,10,00,79,10,00,80,10,00,81,10,00,82,10,00,83,10,00$, $84,10,00,85,10,00,86,10,00,87,10,00,88,10,00,89,10,00$, $90,10,00,91,10,00,92,10,00,93,10,00,94,10,00,95,10,00$, $96,10,00,97,10,00,98,10,00,99,10,00,100,10,00,101,10,00$, $102,10,00,103,10,00,104,10,00,105,10,00,106,10,00,107,10,00$, $108,10,00,109,10,00,110,10,00,111,10,00$,

$I^{*}-$ LRU \#3 -Port 4 (Analog)

$0,10,00,1,10,00,2,10,00,3,10,00,4,10,00,5,10,00$, $6,10,00,7,10,00,8,10,00,9,10,00,10,10,00,11,10,00$, $12,10,00,13,10,00,14,10,00,15,10,00,16,10,00,17,10,00$, $18,10,00,19,10,00,20,10,00,21,10,00,22,10,00,23,10,00$, $24,10,00,25,10,00,26,10,00,27,10,00,28,10,00,29,10,00$, $30,10,00,31,10,00,32,10,00,33,10,00,34,10,00,35,10,00$, $36,10,00,37,10,00,38,10,00,39,10,00,40,10,00,41,10,00$, $42,10,00,43,10,00,44,10,00,45,10,00,46,10,00,47,10,00$, $48,10,00,49,10,00,50,10,00,51,10,00,52,10,00,53,10,00$, $54,10,00,55,10,00,56,10,00,57,10,00,58,10,00,59,10,00$, $60,10,00,61,10,00,62,10,00,63,10,00,64,10,00,65,10,00$, $66,10,00,67,10,00,68,10,00,69,10,00,70,10,00,71,10,00$, $72,10,00,73,10,00,74,10,00,75,10,00,76,10,00,77,10,00$, $78,10,00,79,10,00,80,10,00,81,10,00,82,10,00,83,10,00$, $84,10,00,85,10,00,86,10,00,87,10,00,88,10,00,89,10,00$, $90,10,00,91,10,00,92,10,00,93,10,00,94,10,00,95,10,00$, $96,10,00,97,10,00,98,10,00,99,10,00,100,10,00,101,10,00$, $102,10,00,103,10,00,104,10,00,105,10,00,106,10,00,107,10,00$, $108,10,00,109,10,00,110,10,00,111,10,00$, 


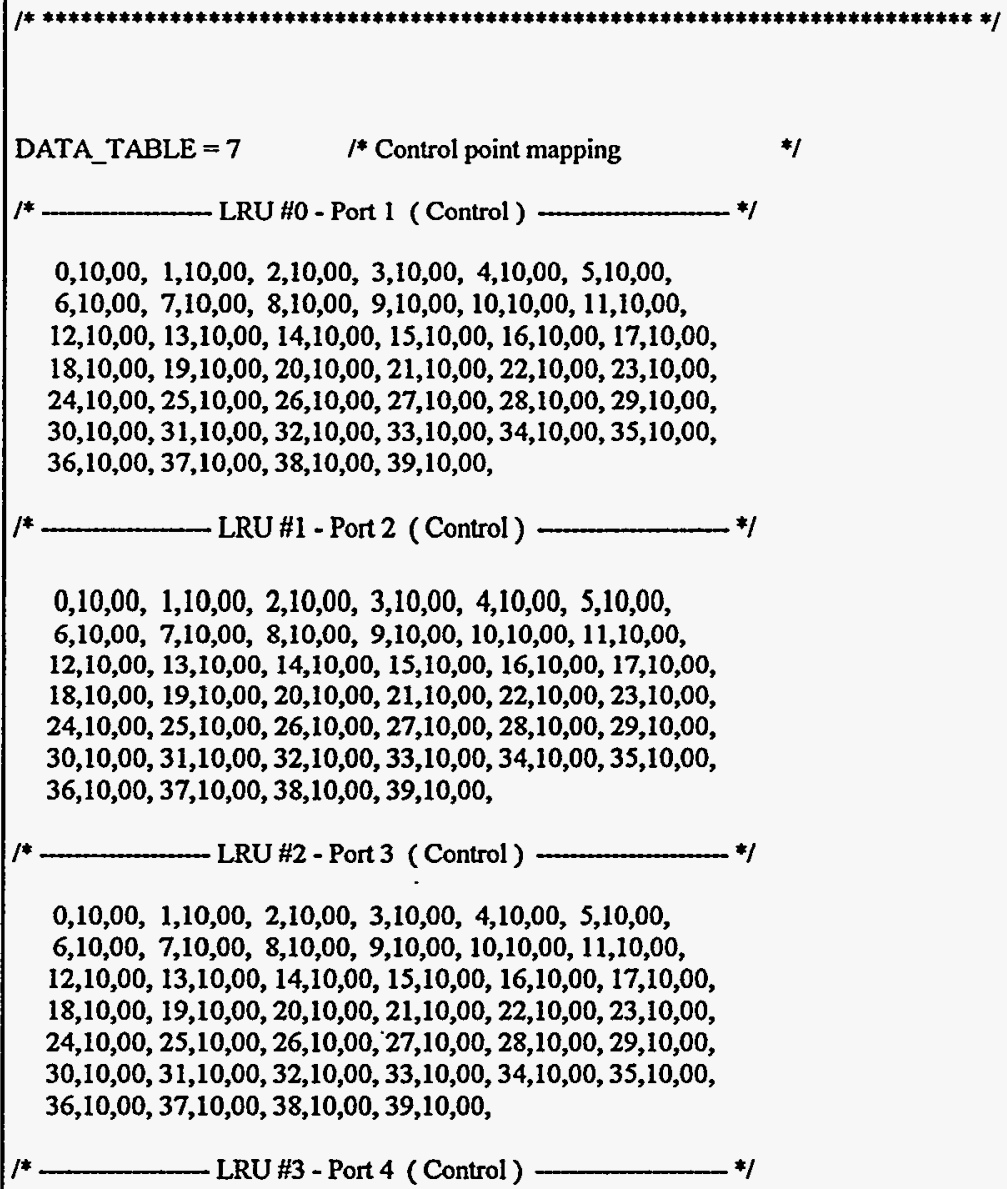

$0,10,00,1,10,00,2,10,00,3,10,00,4,10,00,5,10,00$, $6,10,00,7,10,00,8,10,00,9,10,00,10,10,00,11,10,00$, $12,10,00,13,10,00,14,10,00,15,10,00,16,10,00,17,10,00$, $18,10,00,19,10,00,20,10,00,21,10,00,22,10,00,23,10,00$, $24,10,00,25,10,00,26,10,00,27,10,00,28,10,00,29,10,00$, $30,10,00,31,10,00,32,10,00,33,10,00,34,10,00,35,10,00$, $36,10,00,37,10,00,38,10,00,39,10,00$,

I* - LRU \#1 - Port 2 (Control) —_ـ

$0,10,00,1,10,00,2,10,00,3,10,00,4,10,00,5,10,00$ $6,10,00,7,10,00,8,10,00,9,10,00,10,10,00,11,10,00$, $12,10,00,13,10,00,14,10,00,15,10,00,16,10,00,17,10,00$, $18,10,00,19,10,00,20,10,00,21,10,00,22,10,00,23,10,00$, $24,10,00,25,10,00,26,10,00,27,10,00,28,10,00,29,10,00$, $30,10,00,31,10,00,32,10,00,33,10,00,34,10,00,35,10,00$, $36,10,00,37,10,00,38,10,00,39,10,00$,

$f^{*}+$ LRU \#2 - Port 3 (Control) _

$0,10,00,1,10,00,2,10,00,3,10,00,4,10,00,5,10,00$, $6,10,00,7,10,00,8,10,00,9,10,00,10,10,00,11,10,00$, $12,10,00,13,10,00,14,10,00,15,10,00,16,10,00,17,10,00$, $18,10,00,19,10,00,20,10,00,21,10,00,22,10,00,23,10,00$, $24,10,00,25,10,00,26,10,00,27,10,00,28,10,00,29,10,00$, $30,10,00,31,10,00,32,10,00,33,10,00,34,10,00,35,10,00$, $36,10,00,37,10,00,38,10,00,39,10,00$,

$l^{*} \longrightarrow$ LRU \#3 - Port 4 (Control) $*$

$0,10,00,1,10,00,2,10,00,3,10,00,4,10,00,5,10,00$, $6,10,00,7,10,00,8,10,00,9,10,00,10,10,00,11,10,00$, $12,10,00,13,10,00,14,10,00,15,10,00,16,10,00,17,10,00$, $18,10,00,19,10,00,20,10,00,21,10,00,22,10,00,23,10,00$, $24,10,00,25,10,00,26,10,00,27,10,00,28,10,00,29,10,00$, $30,10,00,31,10,00,32,10,00,33,10,00,34,10,00,35,10,00$, $36,10,00,37,10,00,38,10,00,39,10,00$,

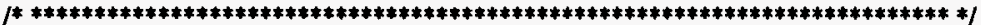

DATA_TABLE $=9 \quad / *$ Accumulator - Form "A" pulse (0 TO 3$) *$

$0,2,1,2,2,2,3,2, \quad / *$ port 1 Normal mode unit (4 pts mapped) *I

$0,2,1,2,2,2,3,2, \quad 1 *$ port 2 Normal mode unit (4 pts mapped) $* 1$

$0,2,1,2,2,2,3,2, \quad 1 *$ port 3 Normal mode unit (4 pts mapped) $* 1$

$0,2,1,2,2,2,3,2, \quad 1 *$ port 4 Normal mode unit (4 pts mapped) */ 


\section{C.3. A026 - Data Translation Application (DTA) Configuration File}

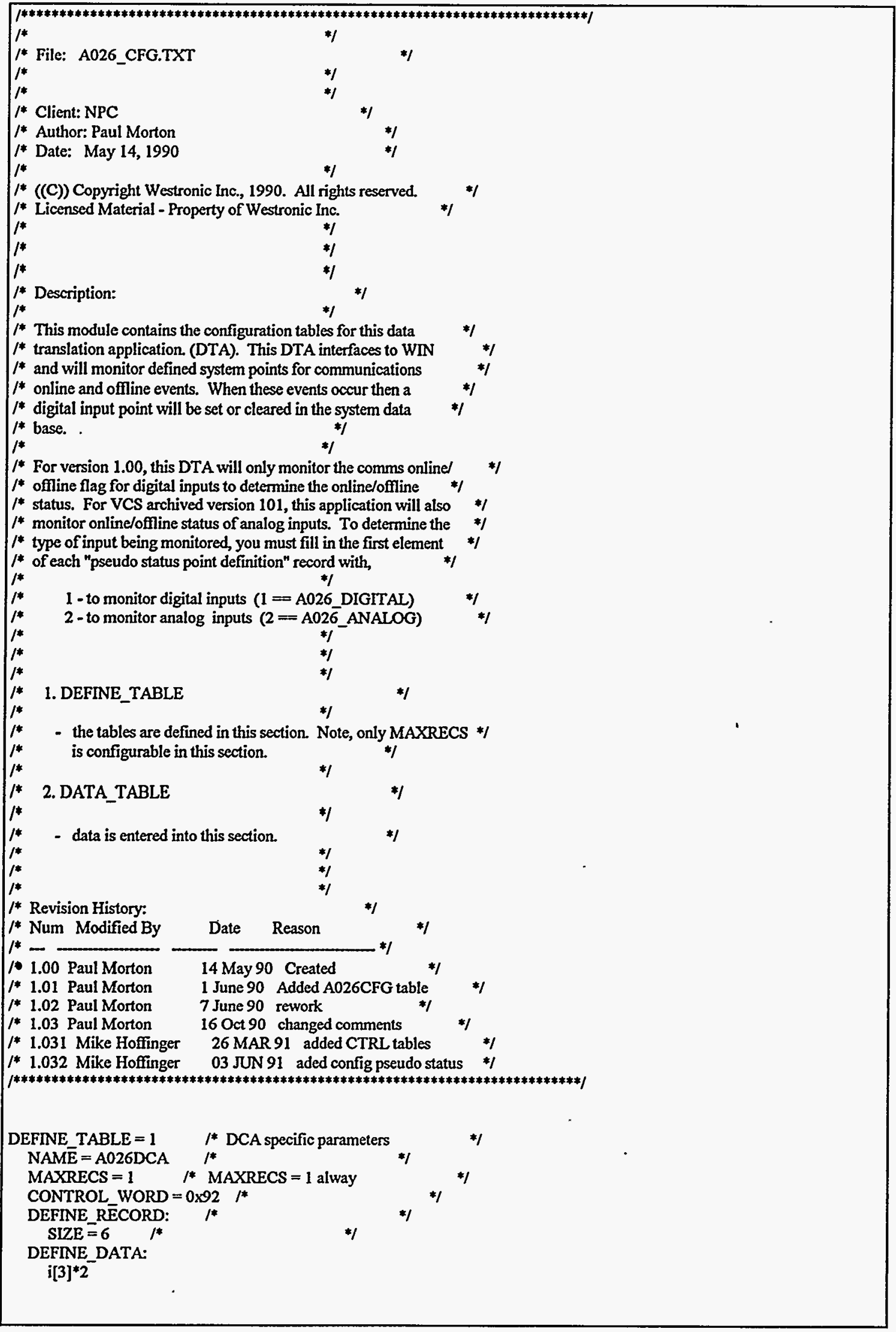




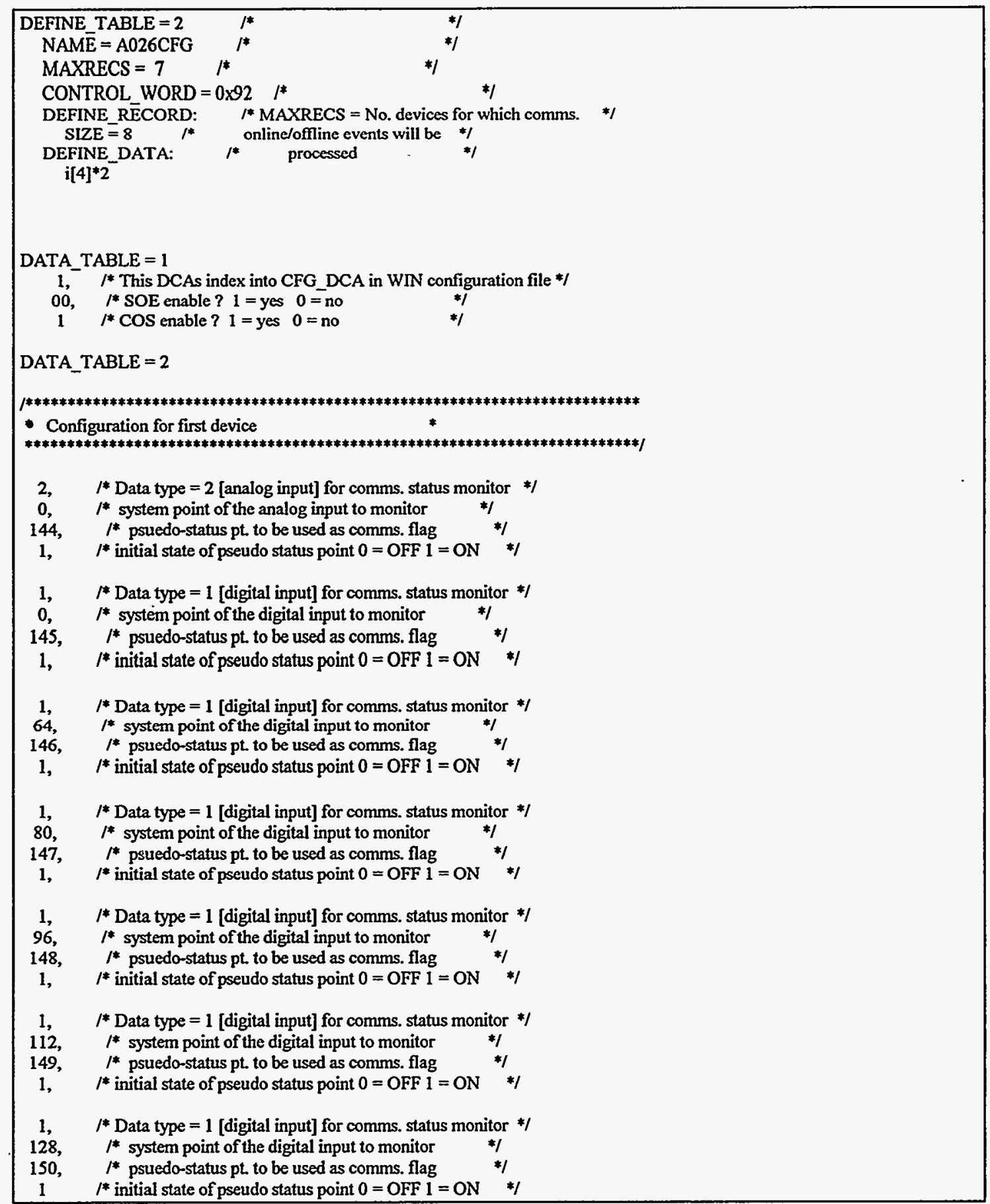




\section{C.4. B003 - D.20 Configuration File}

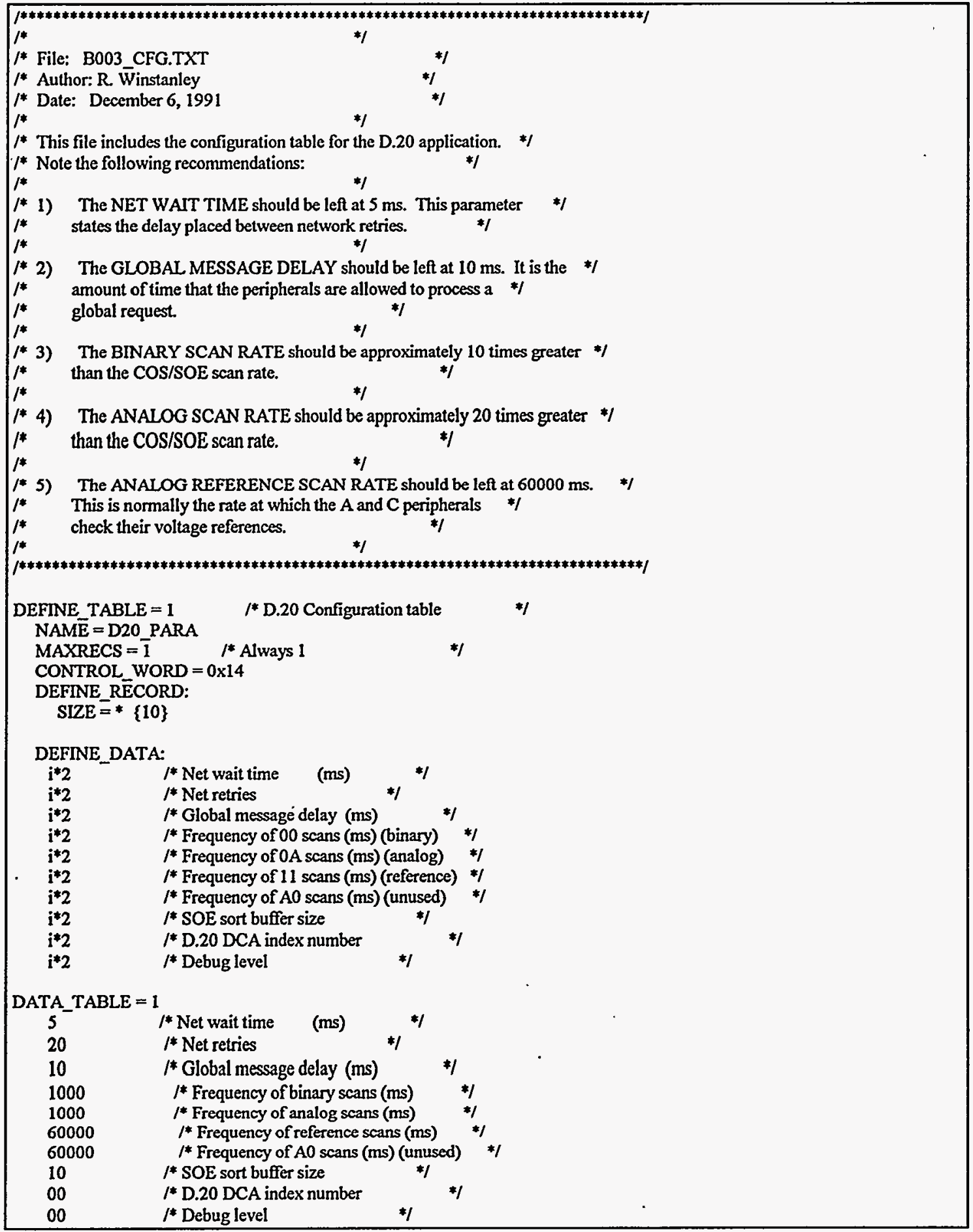




\section{C.5. B008 - Harris/SCADA Configuration File}

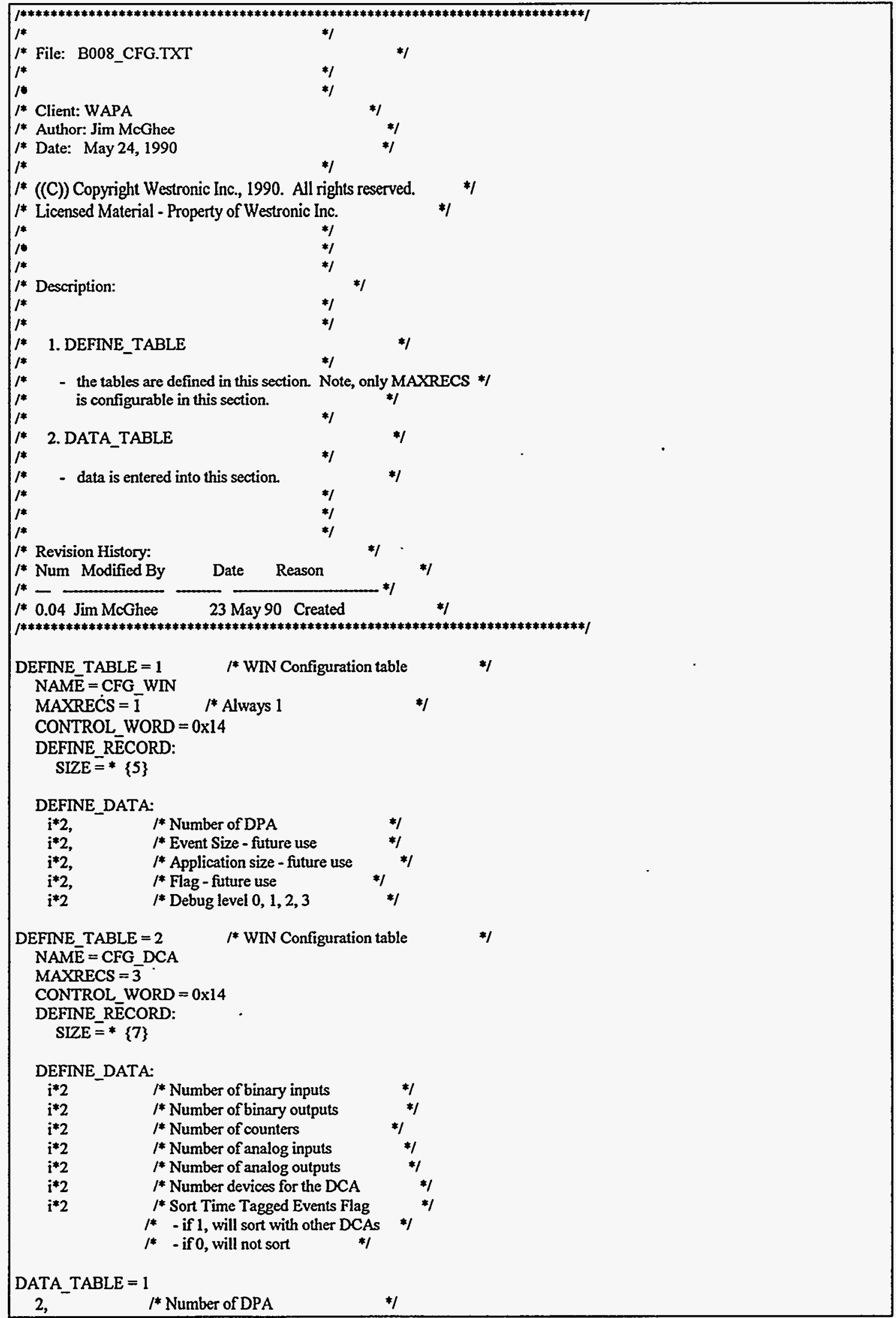




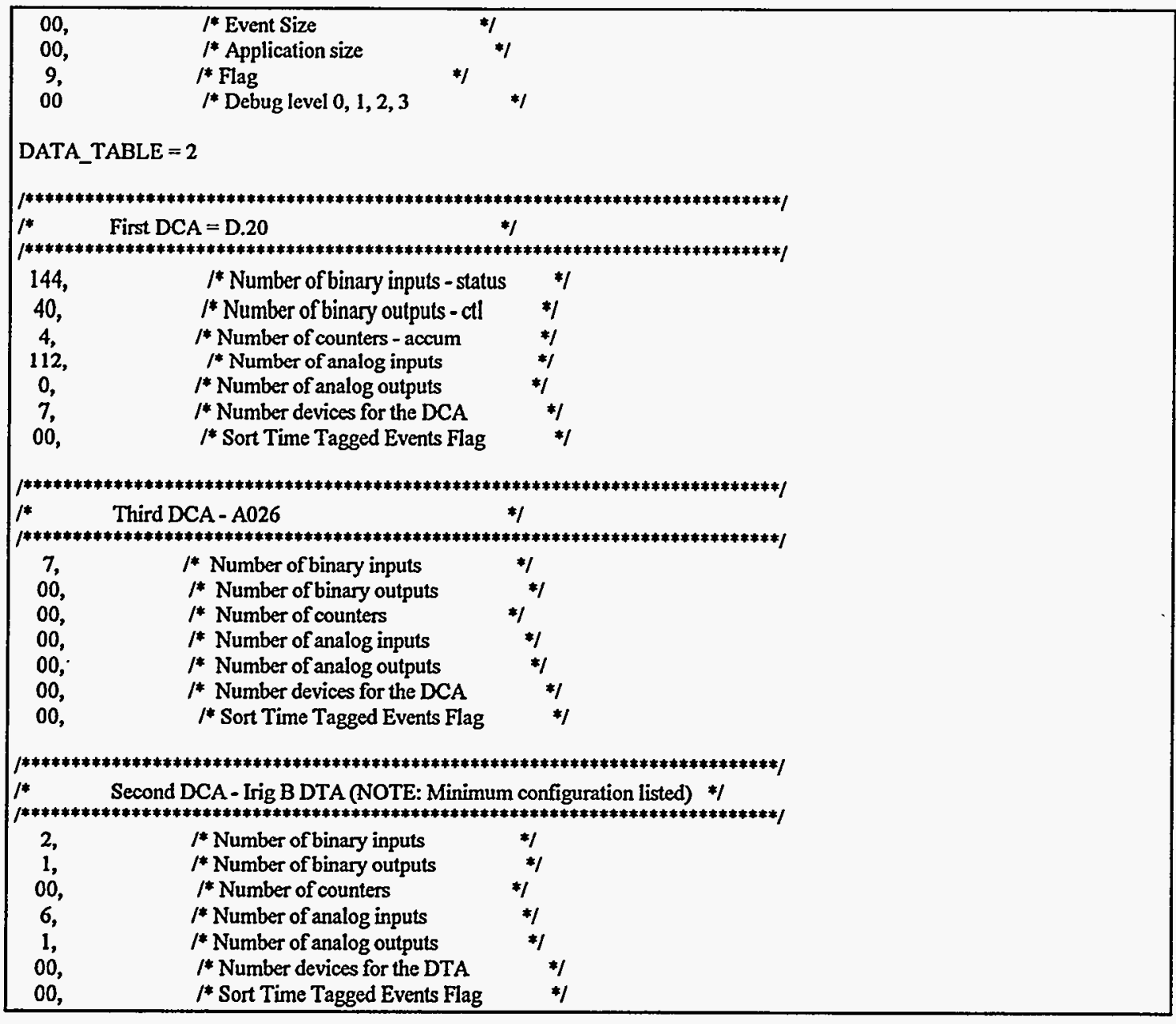




\section{C.6. B012 - IRIG Configuration File}

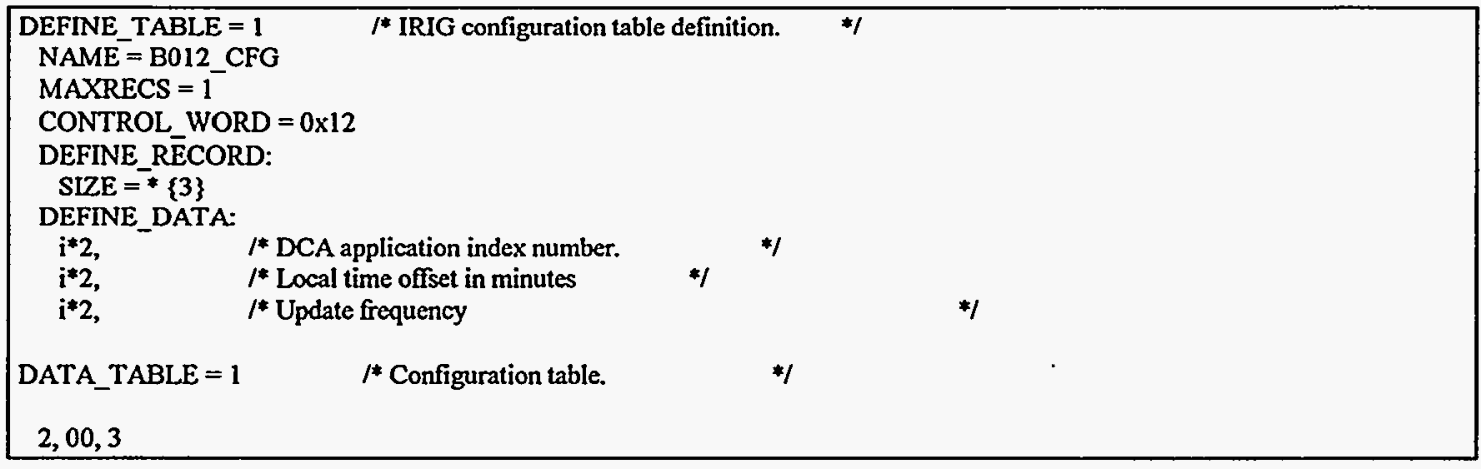


C.7. B014 - Westmaint II Configuration Definition File

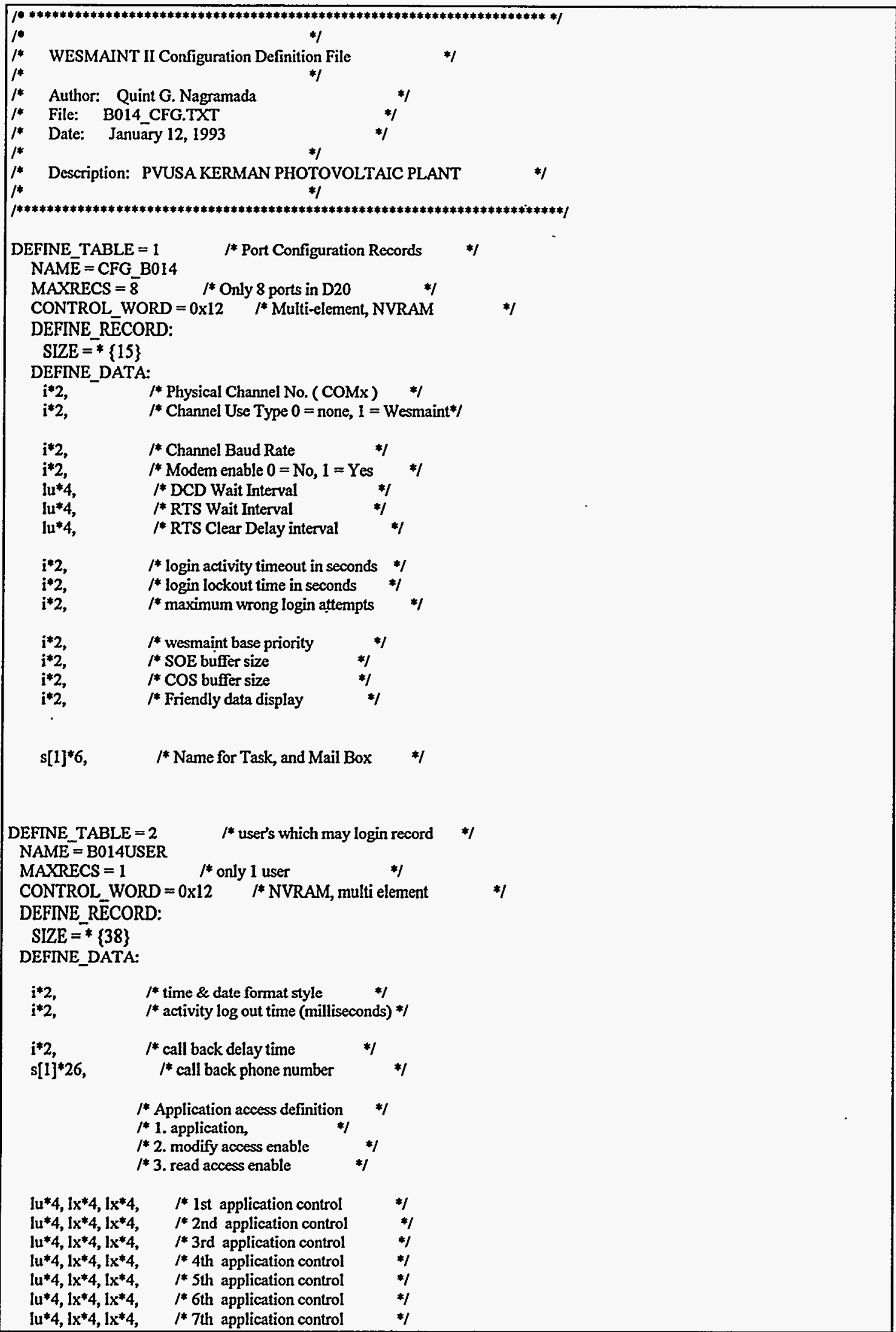




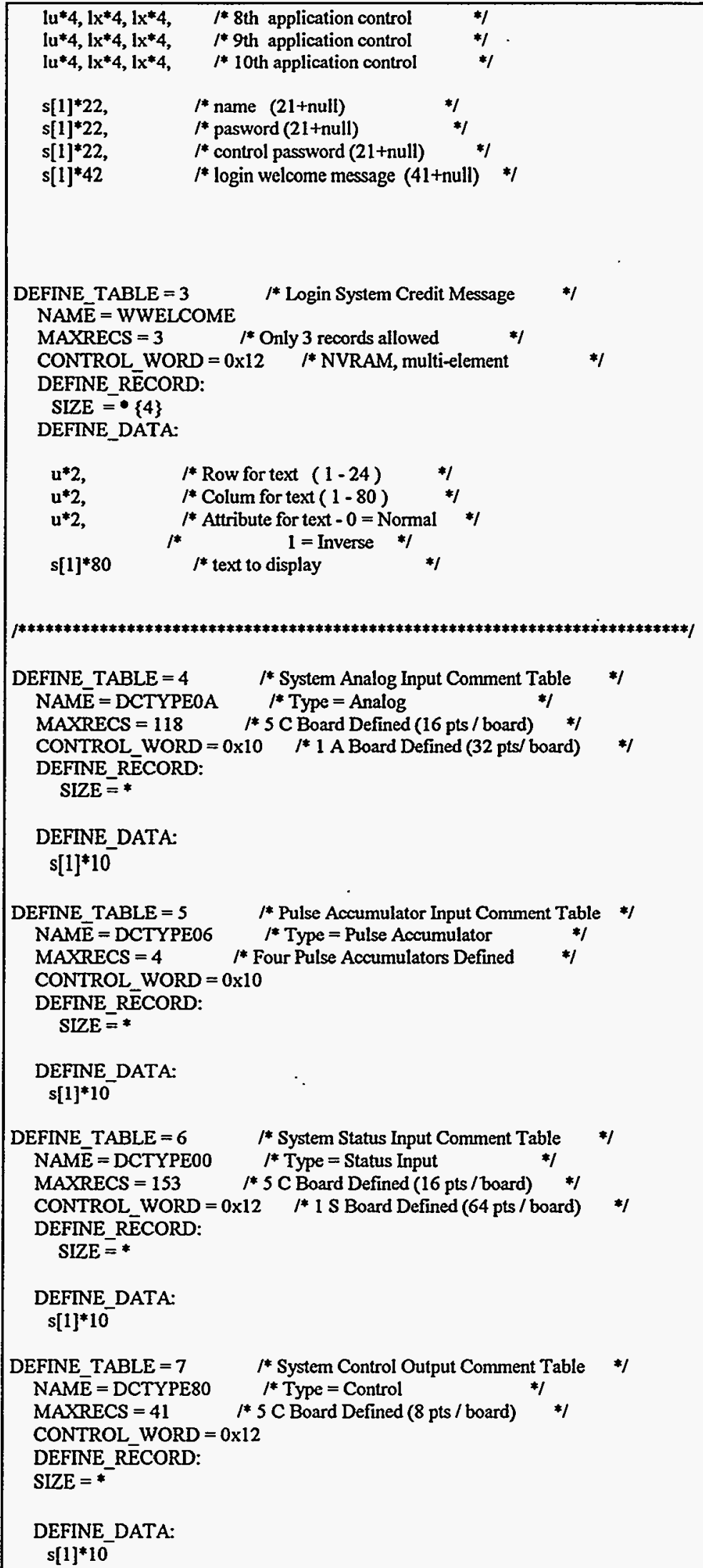




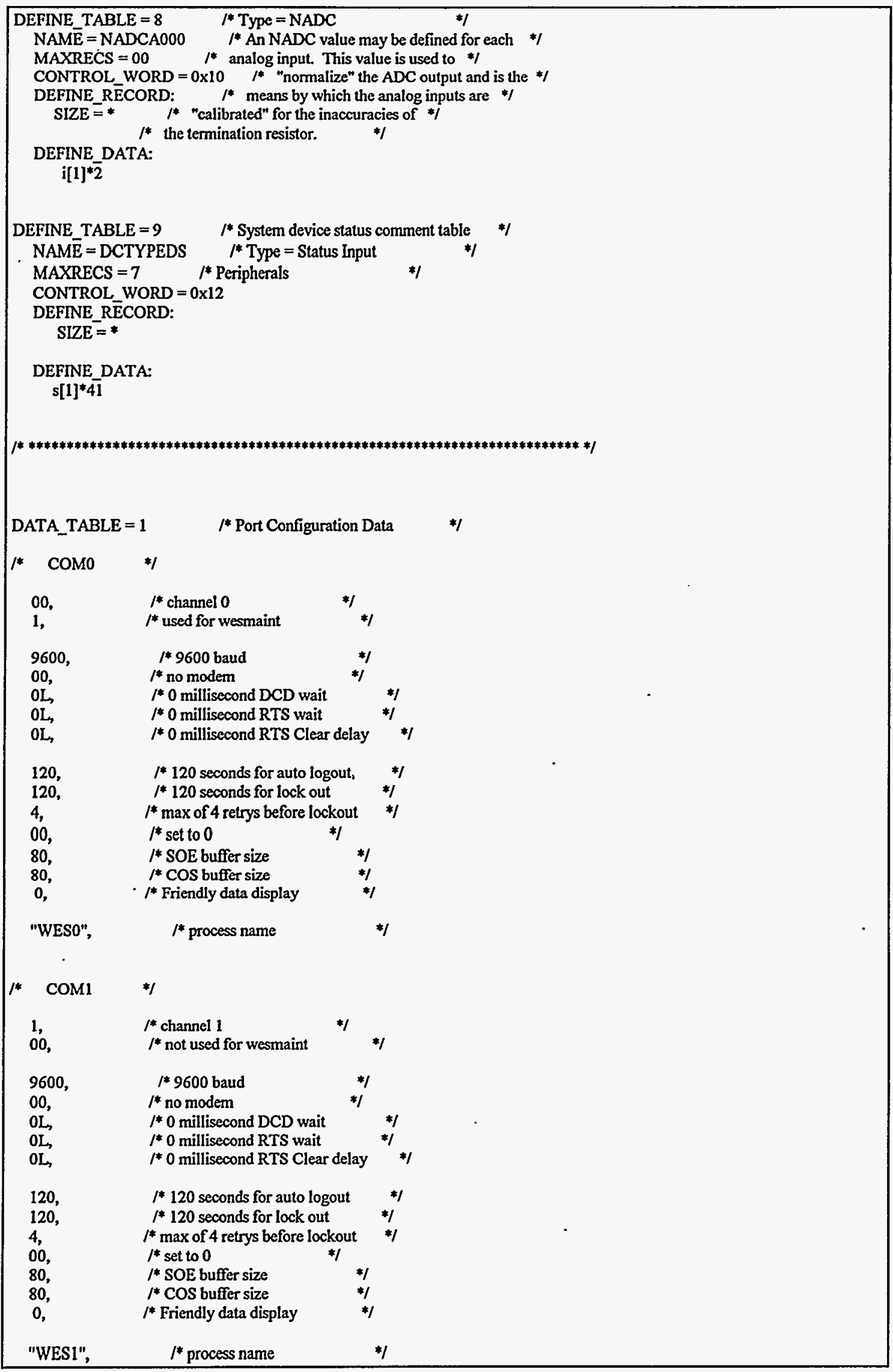




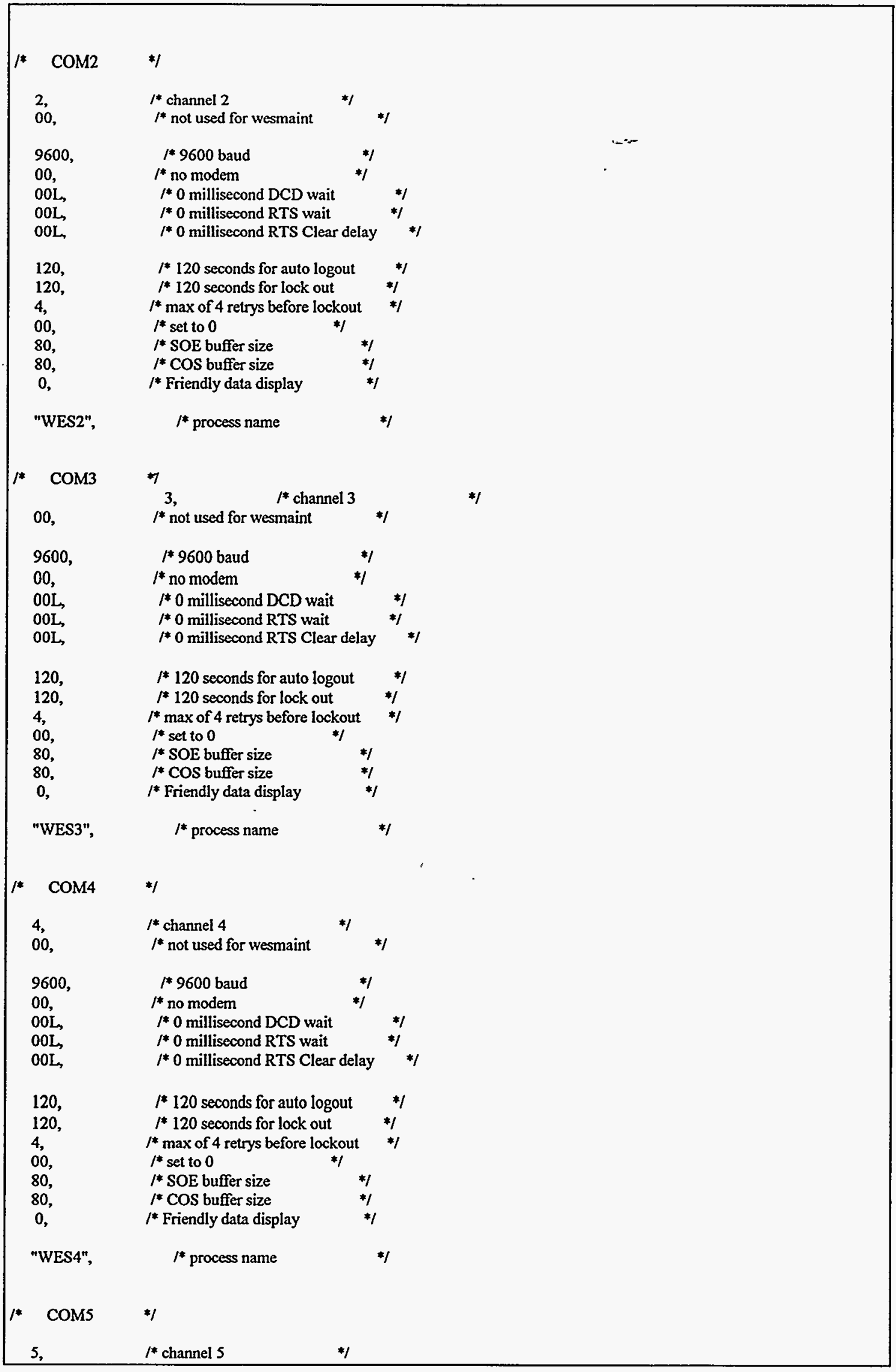




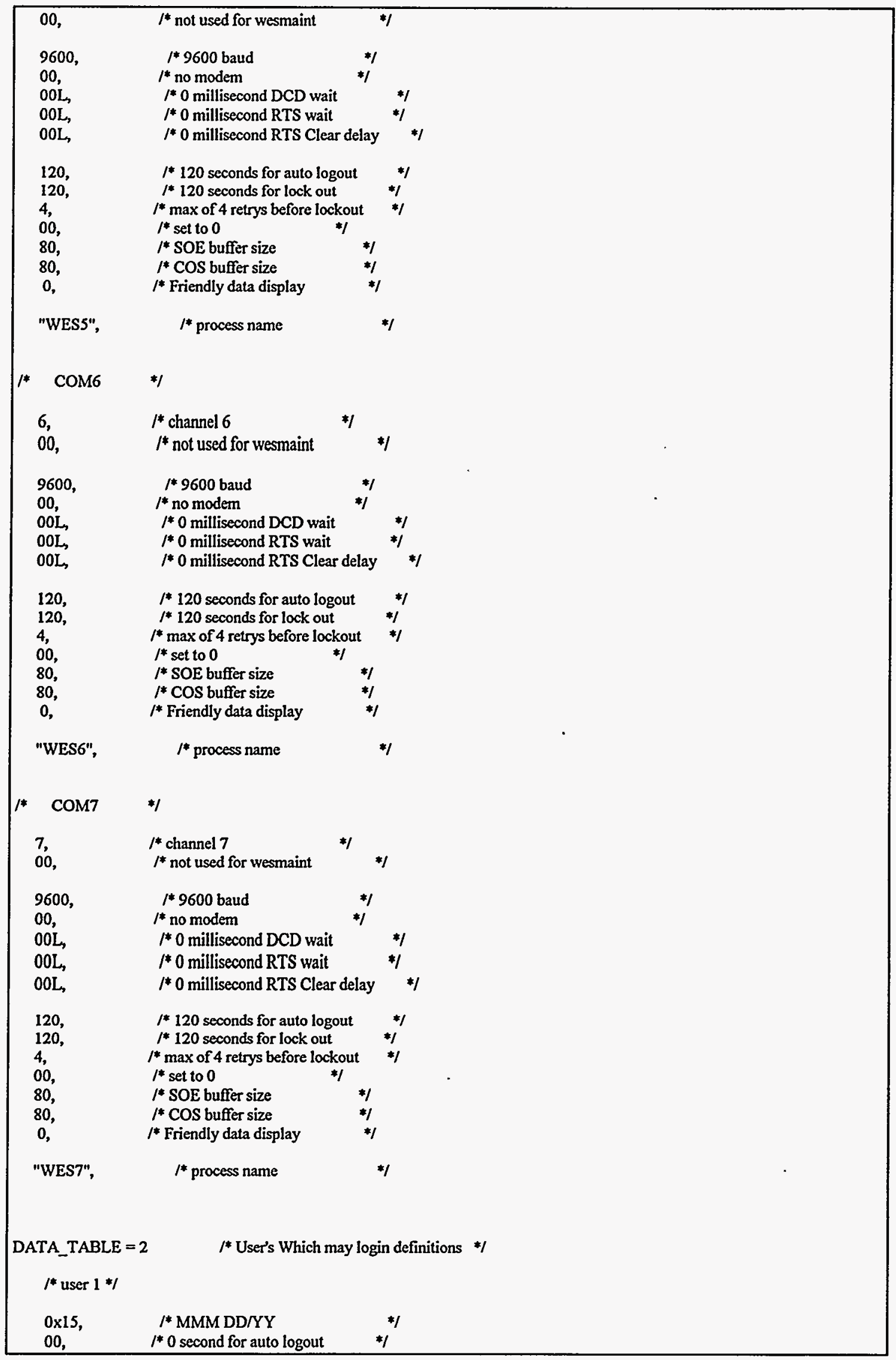




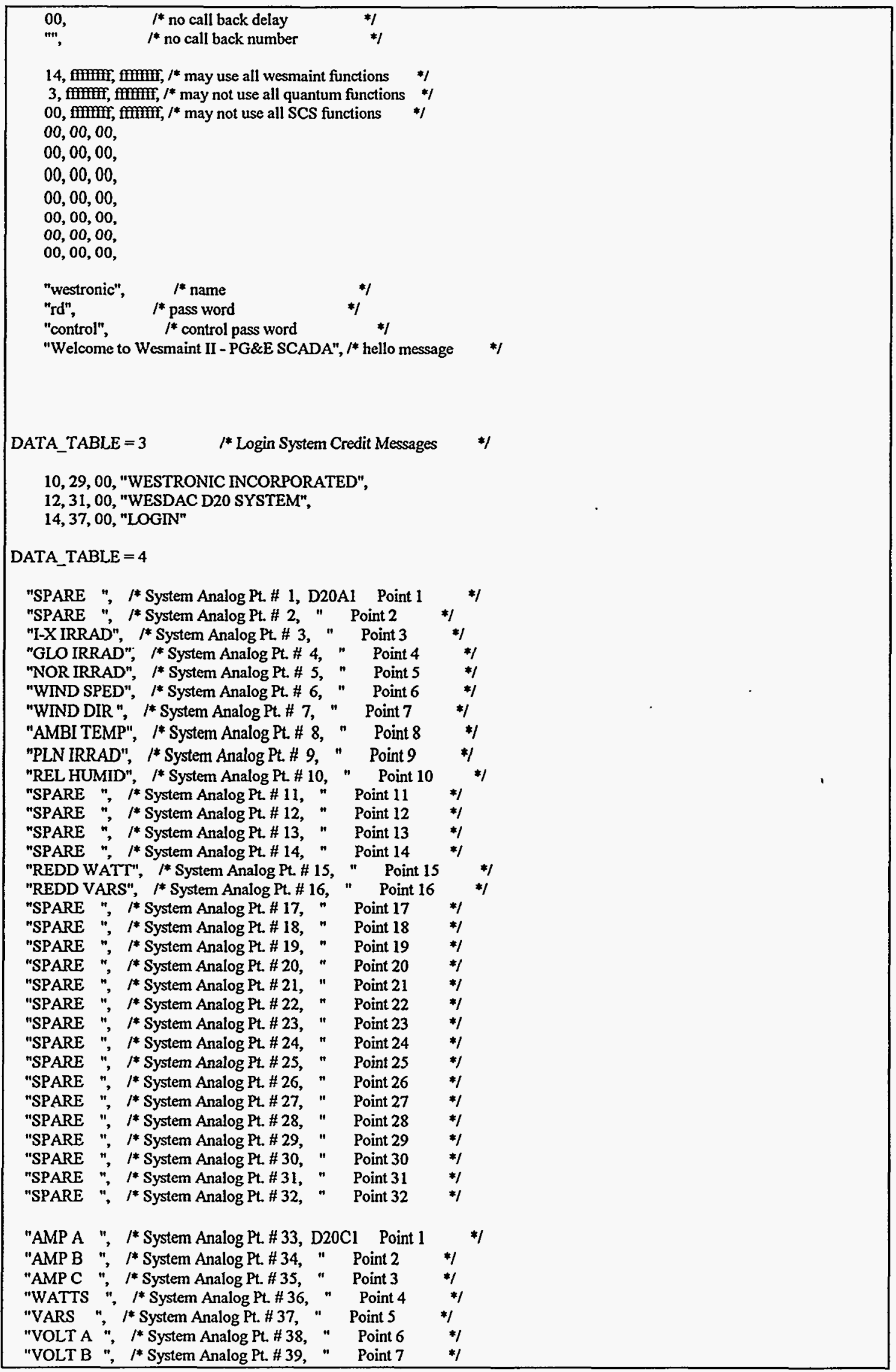




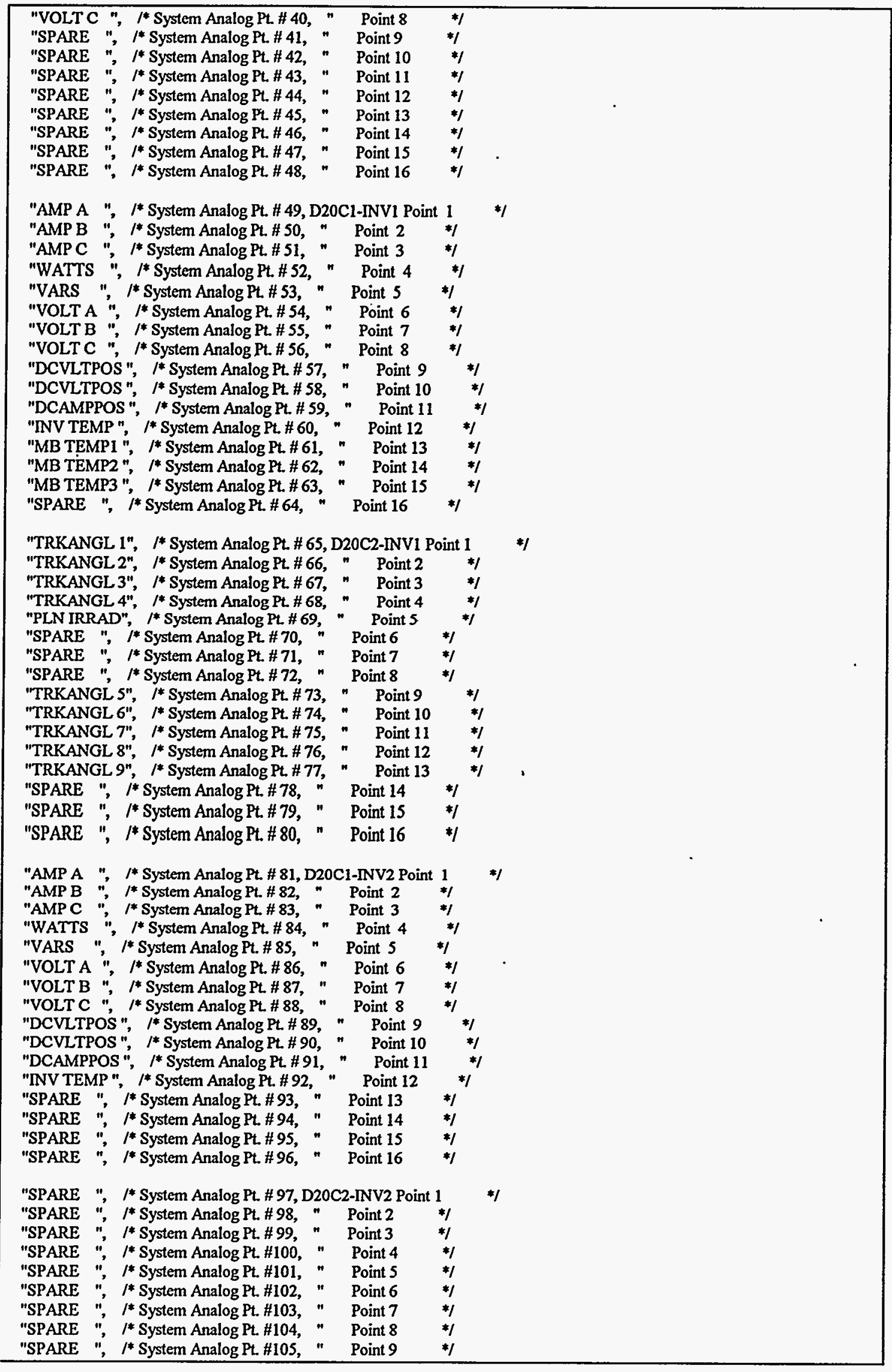




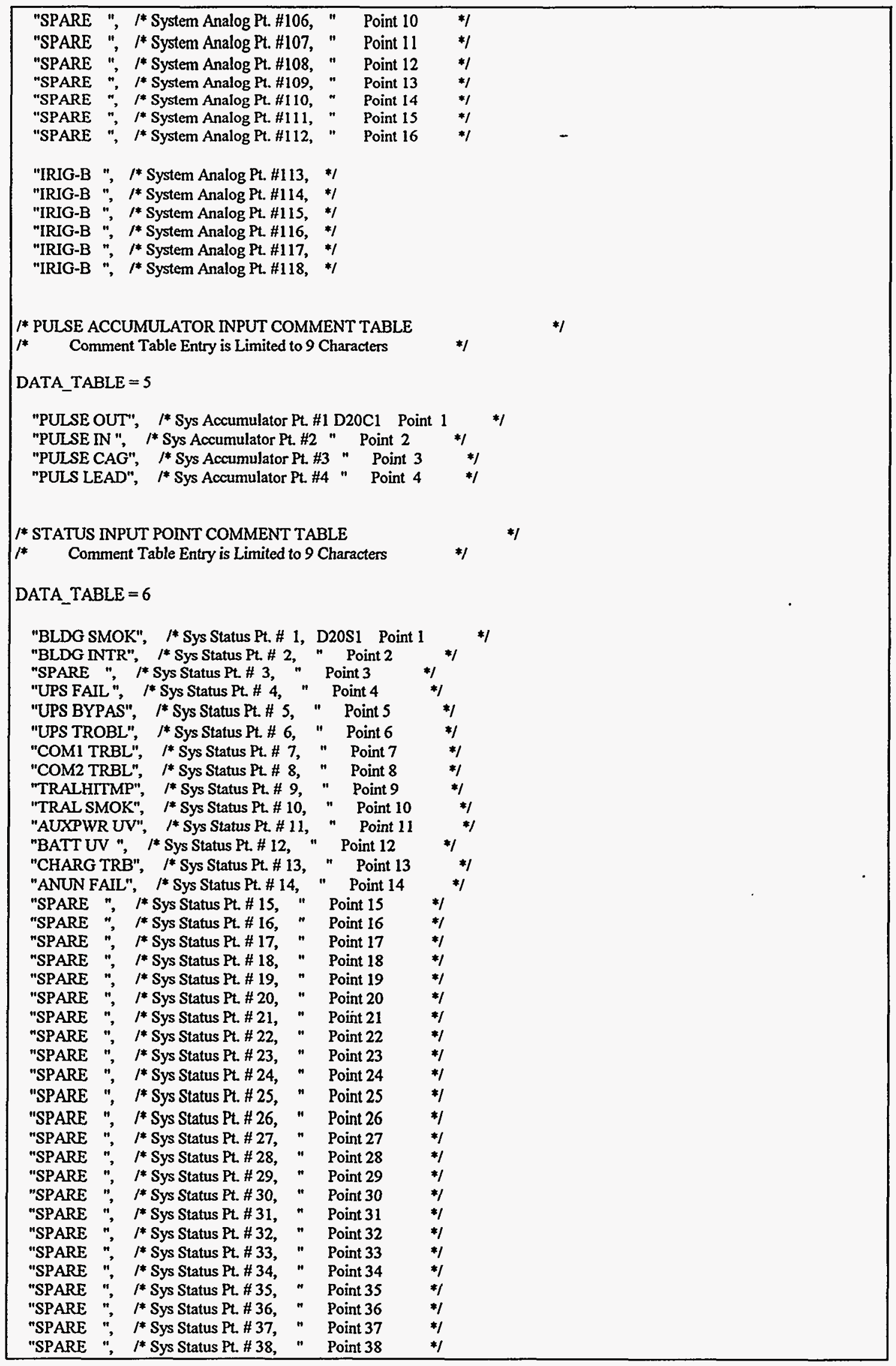




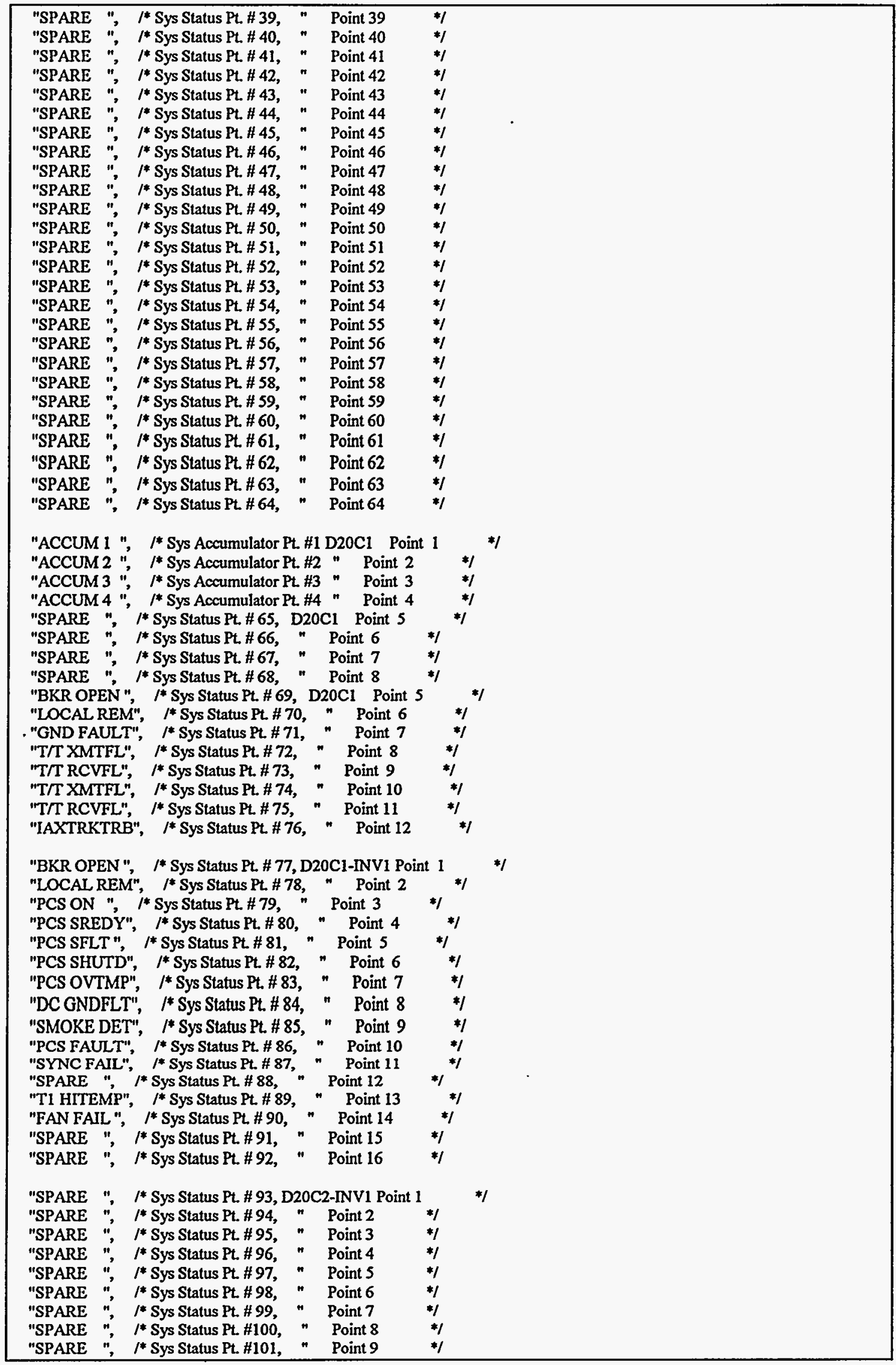




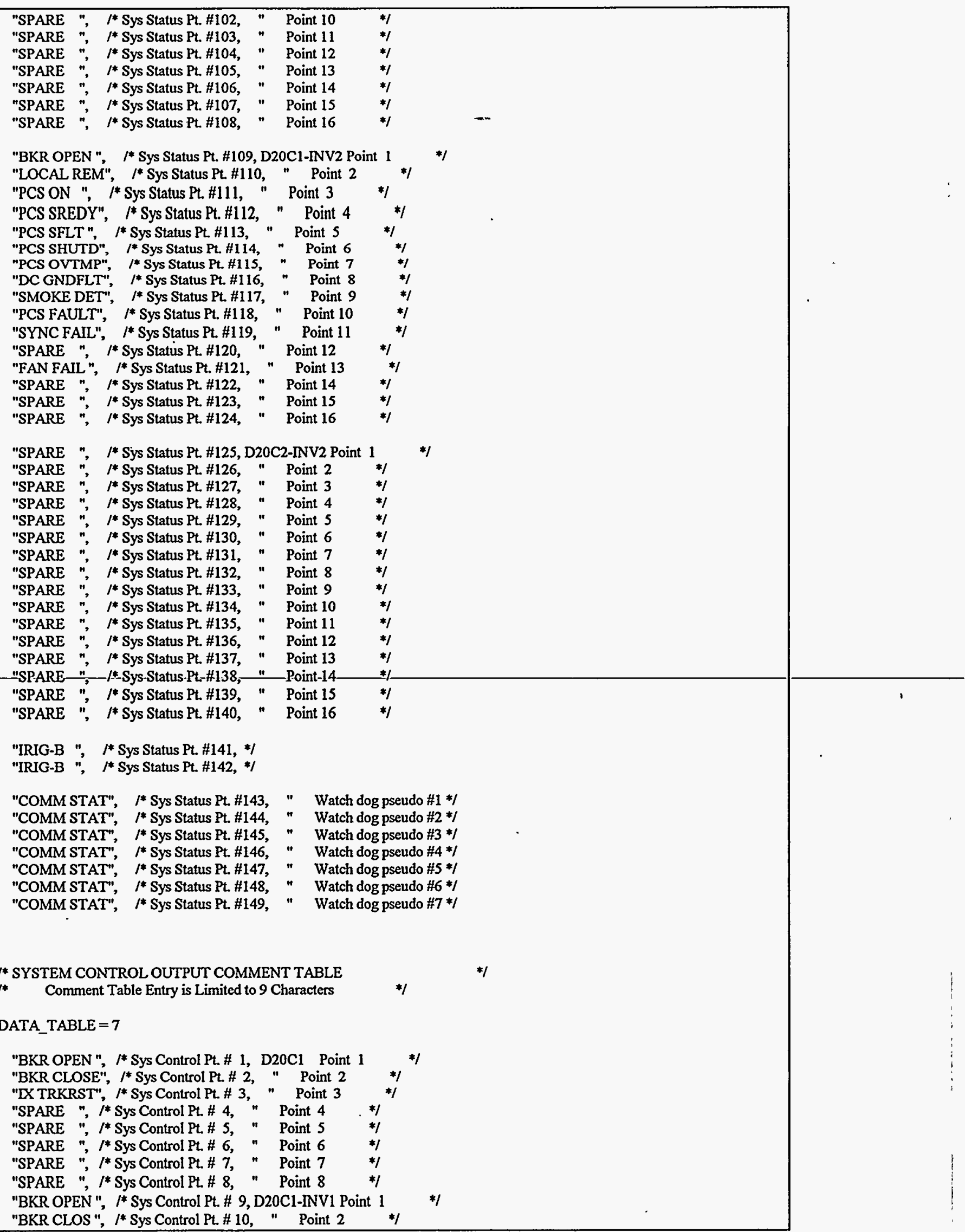

"BKR OPEN", /* Sys Control Pt. \# 9, D20C1-INV1 Point 1
"BKR CLOS", /* Sys Control Pt. \# 10, " Point $2 \quad * /$ 


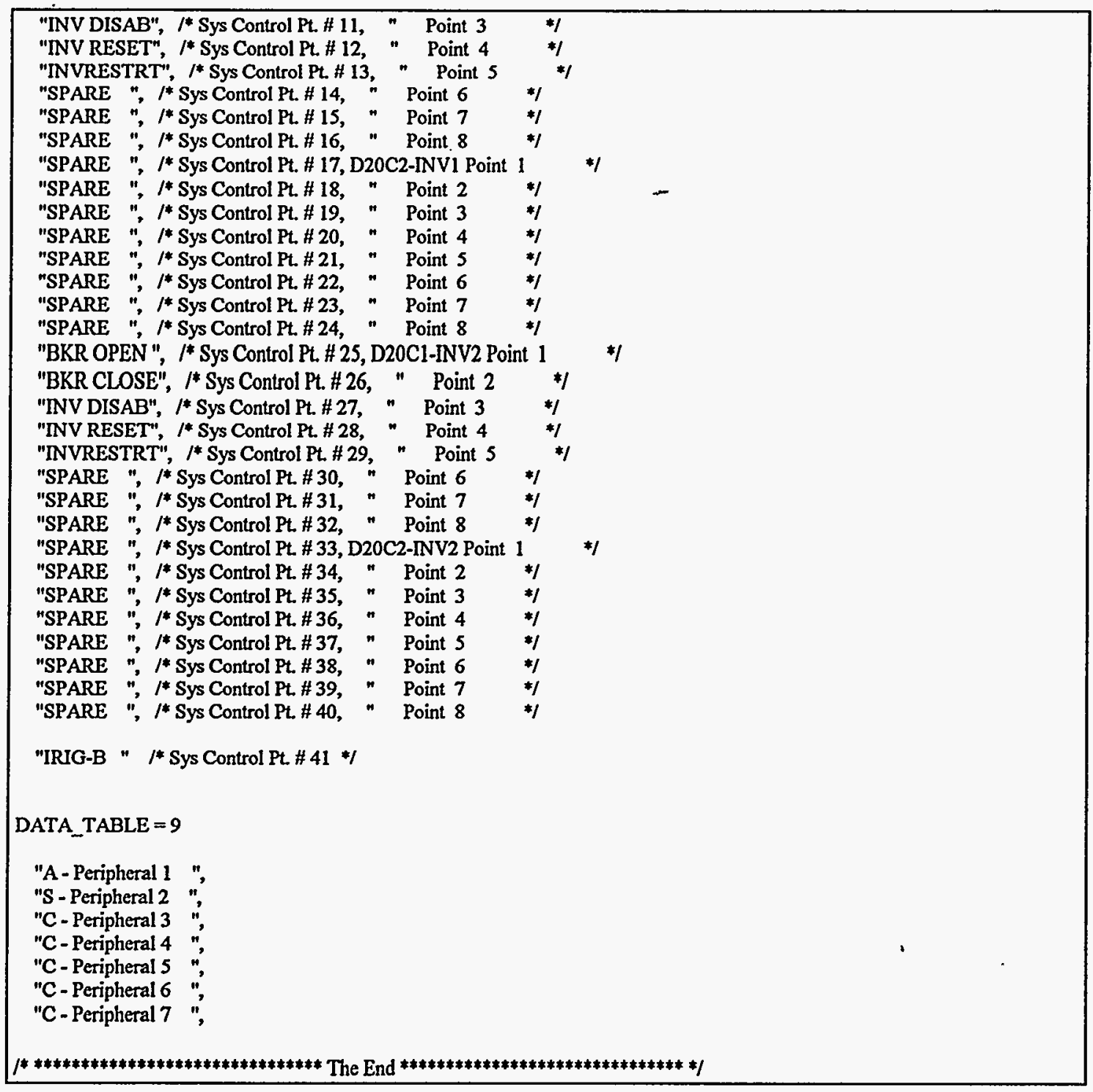


Appendix D

SOFTWARE CONFIGURATION FILES 


\section{SOFTWARE CONFIGURATION FILES}

\section{D.1. QNX}

\section{D.1.1. SYS.INIT.0}

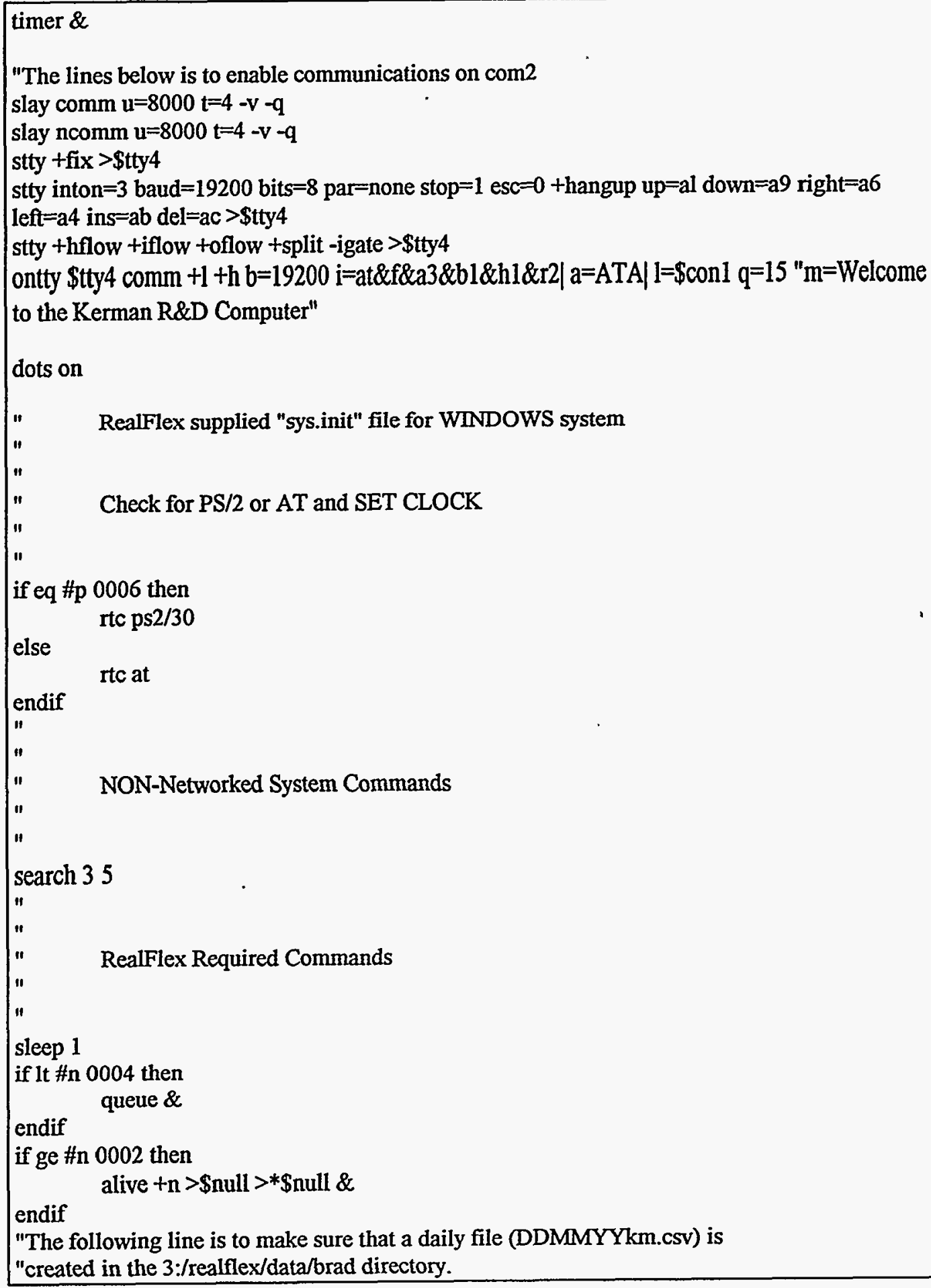




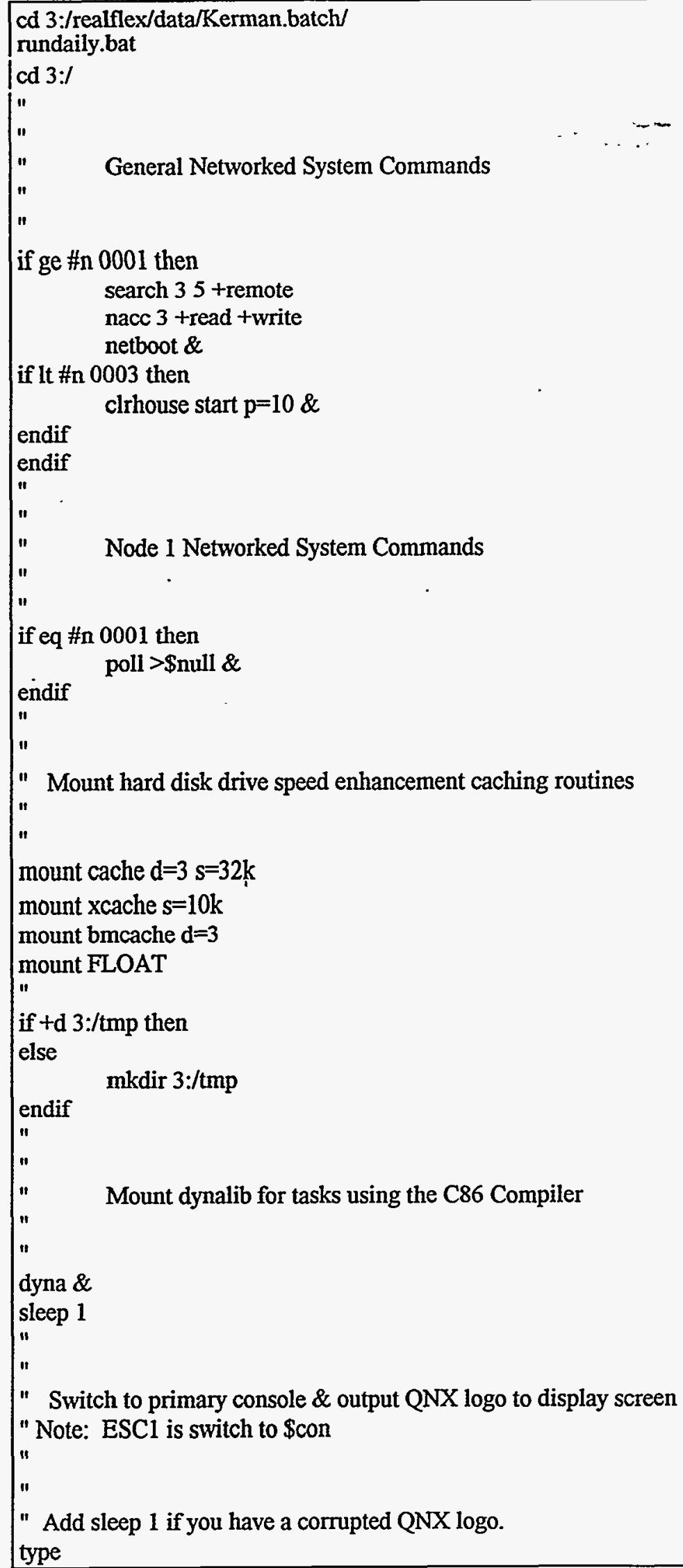




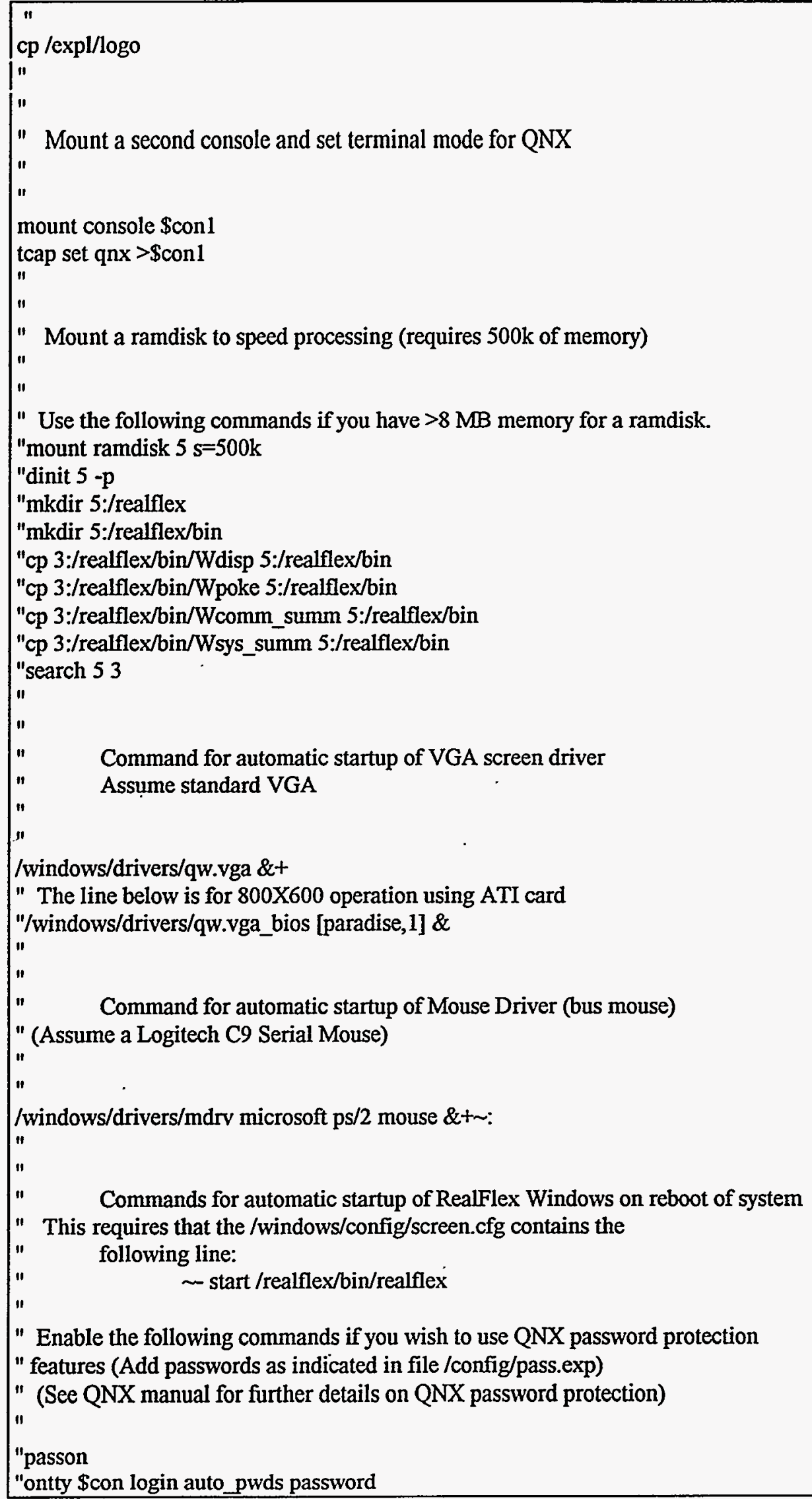




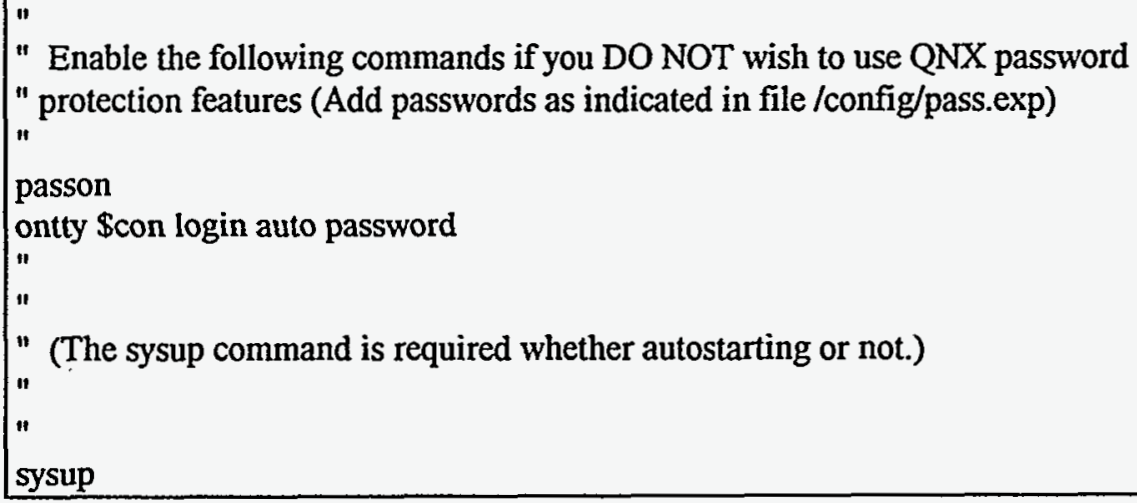

\section{D.2. RealFlex}

\section{D.2.1. COLDSTART}

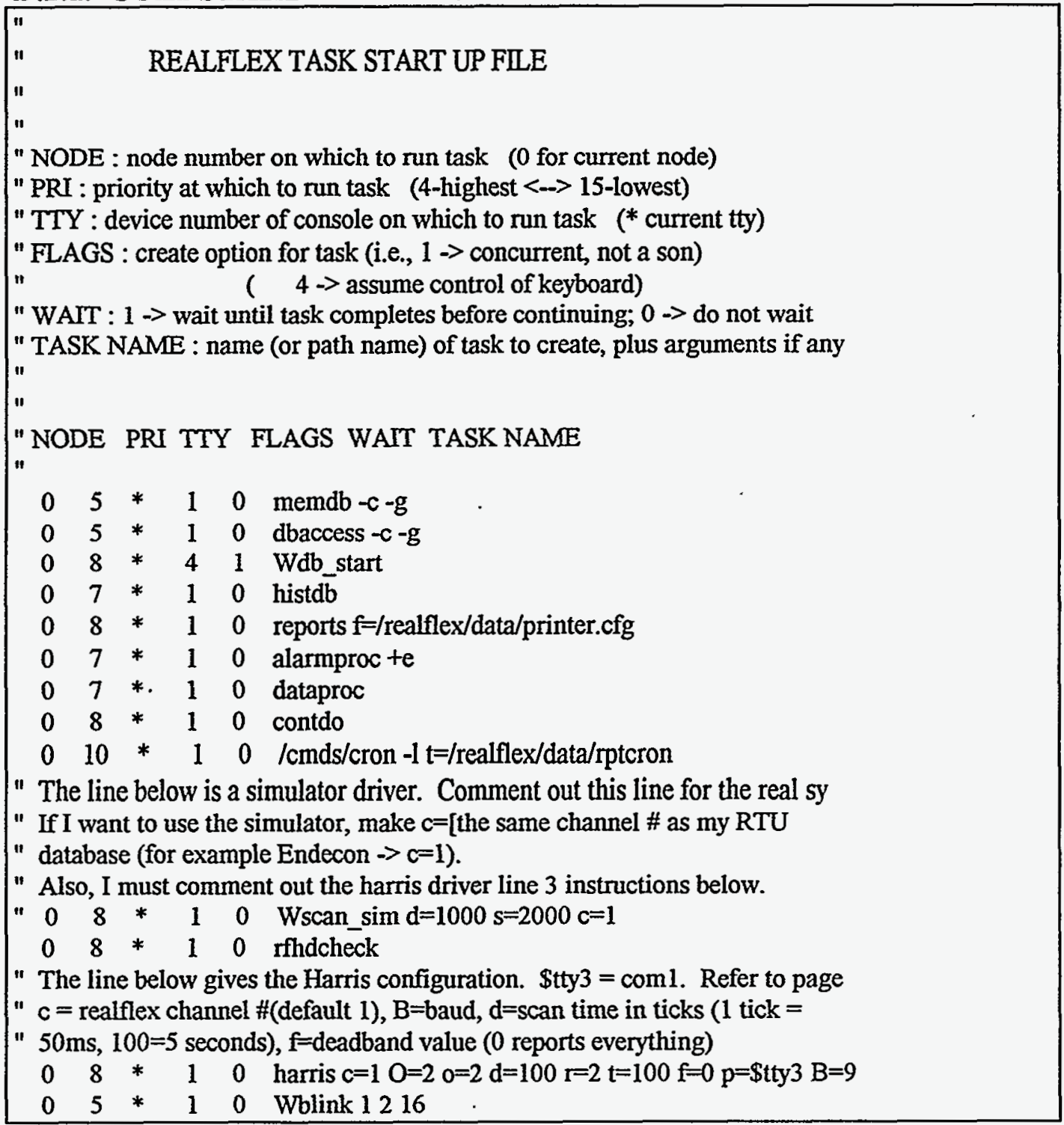




\begin{tabular}{|c|c|c|c|c|}
\hline $\begin{array}{l}0 \\
0\end{array}$ & $\begin{array}{l}8 \\
6\end{array}$ & $\begin{array}{ll}* & 1 \\
* & 1\end{array}$ & $\begin{array}{l}0 \\
0\end{array}$ & $\begin{array}{l}\text { Walm_lines } \\
\text { pass_adm }\end{array}$ \\
\hline 0 & 6 & * 1 & 0 & Wcontrols \\
\hline 0 & 6 & * & 0 & update \\
\hline 0 & 8 & * & 0 & Wfonts \\
\hline 0 & 6 & * & 0 & heapmgr \\
\hline 0 & 6 & * & 0 & textmgr \\
\hline 0 & 9 & * 1 & 0 & action $\mathrm{f}=/ \mathrm{realflex} /$ data/alm_action \\
\hline " 0 & 9 & * 1 & 0 & Wcalcdo/realflex/data/USER_CALC_FILE \\
\hline
\end{tabular}

\section{D.2.2. RPTCRON}

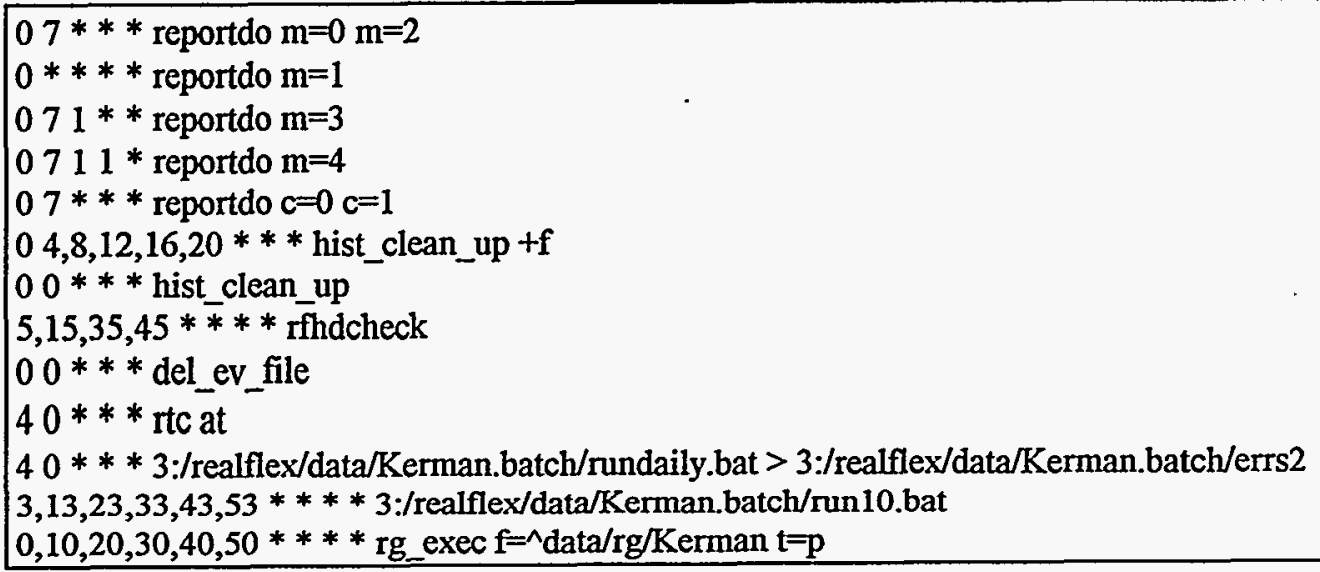


Appendix E

\section{REALFLEX DATABASE CALIBRATION PARAMETERS}




\section{REALFLEX DATABASE CALIBRATION PARAMETERS}

\section{Kerman PV Plant Calibration Factors as of 10/26/93}

\begin{tabular}{|c|c|c|c|c|c|}
\hline TAG NAME & POINT DESC & MIN RAW & MAX RAW & MIN EU & MAX EU \\
\hline D20A1_AN03 & WEATHER - GSA & -2048 & 2047 & -1800 & 1800 \\
\hline D20A1_AN04 & WEATHER - GHI & -2048 & 2047 & -1800 & 1800 \\
\hline D20A1_AN05 & WEATHER - DNI & -2048 & 2047 & -1800 & 1800 \\
\hline D20A1_AN06 & WEATHER - WIND SPED & -2048 & 2047 & -50 & 50 \\
\hline D20A1_AN07 & WEATHER - WIND DIR & 0 & 2047 & 0 & 360 \\
\hline D20AI_AN08 & WEATHER - AMBI TEMP & -2048 & 2047 & -50 & 50 \\
\hline D20A1_AN10 & WEATHER - REL HUMID & 0 & 2047 & 0 & 120 \\
\hline D20A1_AN15 & REDUND - WATTS & -2048 & 2047 & -540 & 540 \\
\hline D20A1_AN16 & REDUND - VARS & -2048 & 2047 & -540 & 540 \\
\hline D20C1-1_AN01 & INV\#1 - AMP A & -2048 & 2047 & -499.47 & 500.625 \\
\hline D20C1-1_AN02 & INV\#1 - AMP B & -2048 & 2047 & -500.806 & 501.343 \\
\hline D20C1-1_AN03 & INVH1 - AMP C & -2048 & 2047 & -499.528 & 500.288 \\
\hline D20C1-1_AN04 & INV\#1 - KWATTS & -2048 & 2047 & -300.680 & 300.270 \\
\hline D20C1-1_AN05 & INV\#1 - KVARS & -2048 & 2047 & -300.706 & 300.564 \\
\hline D20C1-1_AN06 & INV\#1 - VOLT A & -2048 & 2047 & -518.231 & 519.995 \\
\hline D20C1-1_AN07 & INV\#I - VOLT B & -2048 & 2047 & -516.426 & 519.950 \\
\hline D20C1-1_AN08 & INV\#1 - VOLT C & -2048 & 2047 & -516.021 & 519.950 \\
\hline D20C1-1_AN09 & INV\#1 - DCVLTPOS & 0 & 2047 & 0 & 598.347 \\
\hline D20C1-1_AN10 & INV\#I - DCVLTNEG & -2048 & 0 & -599.768 & 0 \\
\hline D20C1-1_AN11 & INV\#1 - DCAMPPOS & -2048 & 2047 & -496.204 & 496.264 \\
\hline D20C1-1_AN13 & INV\#1 - MB TEMPI & 0 & 2047 & -100 & 400 \\
\hline D20C1-1_AN14 & INVH1 - MB TEMP2 & 0 & 2047 & -100 & 400 \\
\hline D20C1-1_AN15 & INV\#1 - MB TEMP3, & 0 & 2047 & -100 & 400 \\
\hline D20C1-2_ANO1 & INV\#2 - AMP A & -2048 & 2047 & -502.626 & 502.515 \\
\hline D20C1-2_AN02 & INV\#2-AMP B & -2048 & 2047 & -502.686 & 502.515 \\
\hline D20C1-2_ANO3 & INV\#2 - AMP C & -2048 & 2047 & -501.796 & 503.206 \\
\hline D20C1-2_AN04 & INV\#2 - KWATTS & -2048 & 2047 & -301.080 & 300.685 \\
\hline D20C1-2_ANOS & INVH2 - KVARS & -2048 & 2047 & -301.281 & 300.942 \\
\hline D20C1-2_AN06 & INV\#2 - VOLT A & -2048 & 2047 & -519.59 & 519.980 \\
\hline D20C1-2_AN07 & INV\#2 - VOLT B & -2048 & 2047 & -520.17 & 520.400 \\
\hline D20C1-2_AN08 & INVH2 - VOLT C & -2048 & 2047 & -520.55 & 520.550 \\
\hline D20C1-2_AN09 & INV\#2 - DCVLTPOS & 0 & 2047 & 0 & 599.886 \\
\hline D20C1-2_AN10 & INV\#2 - DCVLTNEG & -2048 & 0 & -600.155 & 0 \\
\hline D20C1-2_AN11 & INV\#2 - DCAMPPOS & -2048 & 2047 & -498.385 & 497.785 \\
\hline D20C1_AN01 & $12 \mathrm{KV}$ - AMP A & -2048 & 2047 & -25.003 & 24.995 \\
\hline D20C1_AN02 & 12KV-AMP B & -2048 & 2047 & -24.948 & 25.037 \\
\hline D02C1_AN03 & 12KV-AMP C & -2048 & 2047 & -24.943 & 24.983 \\
\hline D20C1_AN04 & 12KV-KWATTS & -2048 & 2047 & -599.2 & 598.934 \\
\hline D20C1_AN05 & $12 \mathrm{KV}$ - KVARS & -2048 & 2047 & -600.9 & 601.552 \\
\hline D20C1_AN06 & $12 \mathrm{KV}$ - VOLT A & -2048 & 2047 & -15547 & 15596 \\
\hline D20C1_AN07 & $12 \mathrm{KV}$ - VOLT B & -2048 & 2047 & -15579 & 15599 \\
\hline D20C1_AN08 & $12 \mathrm{KV}$ - VOLT C & -2048 & 2047 & -15437 & 15576 \\
\hline D20C2-1_AN01 & INV\#1 - TRKANGL 1 & 0 & 2047 & 69.072 & -50.643 \\
\hline D20C2-1_AN02 & INV\#I - TRKANGL 2 & 0 & 2047 & 69.304 & -52.828 \\
\hline D20C2-1_AN03 & INV\#1 - TRKANGL 3 & 0 & 2047 & 71.007 & -52.387 \\
\hline D20C2-1_AN04 & INV\#1 - TRKANGL 4 & 0 & 2047 & 69.768 & -50.912 \\
\hline
\end{tabular}




\begin{tabular}{|llllll|}
\hline D20C2-1_AN05 & INV\#1 - PLN IRRAD & 0 & 2047 & -1800 & 1800 \\
\hline D20C2-1_AN09 & INV\#1 - TRKANGL 5 & 0 & 2047 & 70.978 & -52.514 \\
\hline D20C2-1_AN10 & INV\#1 - TRKANGL 6 & 0 & 2047 & 71.264 & -53.400 \\
\hline D20C2-1_AN11 & INV\#1 - TRKANGL 7 & 0 & 2047 & 70.657 & -55.189 \\
\hline D20C2-1_AN12 & INVH1 - TRKANGL8 & 0 & 2047 & 69.400 & -51.312 \\
\hline D20C2-1_AN13 & INV\#1 - TRKANGL9 & 0 & 2047 & 69.556 & -51.602 \\
\hline
\end{tabular}


Appendix F

\section{KERMAN DATABASE MAPPING}


KERMAN DATABASE MAPPING

\begin{tabular}{|c|c|c|c|c|}
\hline \\
\hline \multicolumn{3}{|c|}{ Pt Offst } & \multicolumn{2}{|l|}{ Aux Address } \\
\hline Port \# & Name & D20 Sys. Point \# & Card Point \# & Card \\
\hline 10 & I-X IRRAD & 3 & 3 & D20Al \\
\hline 10 & GLO IRRAD & 4 & 4 & $\mathrm{D} 20 \mathrm{Al}$ \\
\hline 10 & NOR IRRAD & 5 & 5 & $\mathrm{D} 20 \mathrm{Al}$ \\
\hline 10 & WIND SPED & 6 & 6 & $\mathrm{D} 20 \mathrm{Al}$ \\
\hline 10 & WIND DIR & 7 & 7 & D20A1 \\
\hline 10 & AMBI TEMP & 8 & 8 & $\mathrm{D} 20 \mathrm{Al}$ \\
\hline 10 & PLN IRRAD & 9 & 9 & $\mathrm{D} 20 \mathrm{Al}$ \\
\hline 10 & REL HUMID & 10 & 10 & D20Al \\
\hline 10 & REDD WATT & 15 & 15 & D20Al \\
\hline 10 & REDD VARS & 16 & 16 & D20Al \\
\hline 11 & AMP A & 33 & 1 & $\mathrm{D} 20 \mathrm{Cl}$ \\
\hline 11 & AMP B & 34 & 2 & $\mathrm{D} 20 \mathrm{Cl}$ \\
\hline 11 & AMP C & 35 & 3 & $\mathrm{D} 20 \mathrm{Cl}$ \\
\hline 11 & WATTS & 36 & 4 & $\mathrm{D} 20 \mathrm{Cl}$ \\
\hline 11 & VARS & 37 & 5 & D20C1 \\
\hline 11 & VOLT A & 38 & 6 & $\mathrm{D} 20 \mathrm{Cl}$ \\
\hline 11 & VOLT B & 39 & 7 & $\mathrm{D} 20 \mathrm{Cl}$ \\
\hline 11 & VOLT C & 40 & 8 & $\mathrm{D} 20 \mathrm{Cl}$ \\
\hline 12 & AMP A & 49 & 1 & D20C1-INV1 \\
\hline 12 & AMP B & 50 & 2 & D20C1-INV1 \\
\hline 12 & $A M P C$ & 51 & 3 & D20C1-INV1 \\
\hline 12 & WATTS & 52 & 4 & D20C1-INV1 \\
\hline 12 & VARS & 53 & 5 & D20C1-INVI \\
\hline 12 & VOLT A & 54 & 6 & D20C1-INV1 \\
\hline 12 & VOLT B & 55 & 7 & D20C1-INV1 \\
\hline 12 & VOLTC & 56 & 8 & D20C1-NNV1 \\
\hline 12 & DCVLTPOS & 57 & 9 & D20C1-INV1 \\
\hline 12 & DCVLTPOS & 58 & 10 & D20C1-INV1 \\
\hline 12 & DCAMPPOS & 59 & 11 & D20C1-INV1 \\
\hline 12 & INV TEMP & 60 & 12 & D20C1-INV1 \\
\hline 12 & MB TEMPI & 61 & 13 & D20C1-INV1 \\
\hline 12 & MB TEMP2 & 62 & 14 & D20C1-INV1 \\
\hline 12 & MB TEMP3 & 63 & 15 & D20C1-INV1 \\
\hline 13 & TRKANGL 1 & 65 & 1 & D20C2-INV1 \\
\hline 13 & TRKANGL 2 & 66 & 2 & D20C2-INV1 \\
\hline 13 & TRKANGL 3 & 67 & 3 & D20C2-INV1 \\
\hline 13 & TRKANGL 4 & 68 & 4 & D20C2-INV1 \\
\hline 13 & PLN IRRAD & 69 & 5 & D20C2-INV1 \\
\hline 13 & TRKANGL 5 & 73 & 9 & D20C2-INVI \\
\hline 13 & TRKANGL 6 & 74 & 10 & D20C2-INV1 \\
\hline 13 & TRKANGL 7 & 75 & 11 & D20C2-INV1 \\
\hline
\end{tabular}




\begin{tabular}{|c|c|c|c|c|}
\hline$\| 13$ & TRKANGL 8 & 76 & 12 & D20C2-INV1 \\
\hline 13 & TRKANGL 9 & 77 & 13 & D20C2-INV1 \\
\hline 14 & AMP A & 81 & 1 & D20C1-INV2 \\
\hline 14 & AMP B & 82 & 2 & D20C1-INV2 \\
\hline 14 & AMP C & 83 & 3 & D20C1-INV2 \\
\hline 14 & WATTS & 84 & 4 & D20C1-INV2 \\
\hline 14 & VARS & 85 & 5 & D20C1-INV2 \\
\hline 14 & VOLT A & 86 & 6 & D20C1-INV2 \\
\hline 14 & VOLT B & 87 & 7 & D20C1-INV2 \\
\hline 14 & VOLT C & 88 & 8 & D20C1-INV2 \\
\hline 14 & DCVLTPOS & 89 & 9 & D20C1-INV2 \\
\hline 14 & DCVLTPOS & 90 & 10 & D20C1-INV2 \\
\hline 14 & DCAMPPOS & 91 & 11 & D20C1-INV2 \\
\hline 14 & INV TEMP & 92 & 12 & D20C1-INV2 \\
\hline \multicolumn{5}{|c|}{ Status Mapping } \\
\hline Pt Offst & & & Aux Address & \\
\hline Port \# & Name & D20 Sys. Point \# & Card Point \# & Card \\
\hline 1 & BLDG SMOK & 1 & 1 & D20S1 \\
\hline 1 & BLDG INTR & 2 & 2 & D20S1 \\
\hline 1 & UPS FAIL & 4 & 4 & $\mathrm{D} 20 \mathrm{~S} 1$ \\
\hline 1 & UPS BYPAS & 5 & 5 & D20S1 \\
\hline 1 & UPS TROBL & 6 & 6 & D20S1 \\
\hline 1 & COM1 TRBL & 7 & 7 & $\mathrm{D} 20 \mathrm{S1}$ \\
\hline 1 & COM2 TRBL & 8 & 8 & D20S1 \\
\hline 1 & TRALHITMP & 9 & 9 & D20S1 \\
\hline 1 & TRAL SMOK & 10 & 10 & D20S1 \\
\hline 1 & AUXPWR UV & 11 & 11 & D20S1 \\
\hline 1 & BATT UV & 12 & 12 & D20S1 \\
\hline 1 & CHARG TRB & 13 & 13 & D20S1 \\
\hline 1 & ANUN FAIL & 14 & 14 & D20S1 \\
\hline 3 & BKR OPEN & 69 & 9 & $\mathrm{D} 20 \mathrm{Cl}$ \\
\hline 3 & LOCAL REM & 70 & 10 & $\mathrm{D} 20 \mathrm{Cl}$ \\
\hline 3 & GND FAULT & 71 & 11 & $\mathrm{D} 20 \mathrm{Cl}$ \\
\hline 3 & T/T XMTFL & 72 & 12 & $\mathrm{D} 20 \mathrm{Cl}$ \\
\hline 3 & T/T RCVFL & 73 & 13 & $\mathrm{D} 20 \mathrm{Cl}$ \\
\hline 3 & T/T XMTFL & 74 & 14 & $\mathrm{D} 20 \mathrm{C} 1$ \\
\hline 3 & T/T RCVFL & 75 & 15 & $\mathrm{D} 20 \mathrm{Cl}$ \\
\hline 3 & IAX TRKTRB & 76 & 16 & $\mathrm{D} 20 \mathrm{Cl}$ \\
\hline 4 & BKR OPEN & 77 & 1 & D20Cl-INV1 \\
\hline 4 & LOCAL REM & 78 & 2 & $\mathrm{D} 20 \mathrm{Cl}-\mathrm{NNV1}$ \\
\hline 4 & PCS ON & 79 & 3 & D20Cl-INVI \\
\hline 4 & PCS SREDY & 80 & 4 & D20C1-INV1 \\
\hline 4 & PCS SFLT & 81 & 5 & D20C1-INV1 \\
\hline 4 & PCS SHUTD & 82 & 6 & D20C1-INV1 \\
\hline 4 & PCS OVTMP & 83 & 7 & D20Cl-INV1 \\
\hline 4 & DC GNDFLT & 84 & 8 & D20C1-INV1 \\
\hline 4 & SMOKE DET & 85 & 9 & D20Ci-INV1 \\
\hline
\end{tabular}




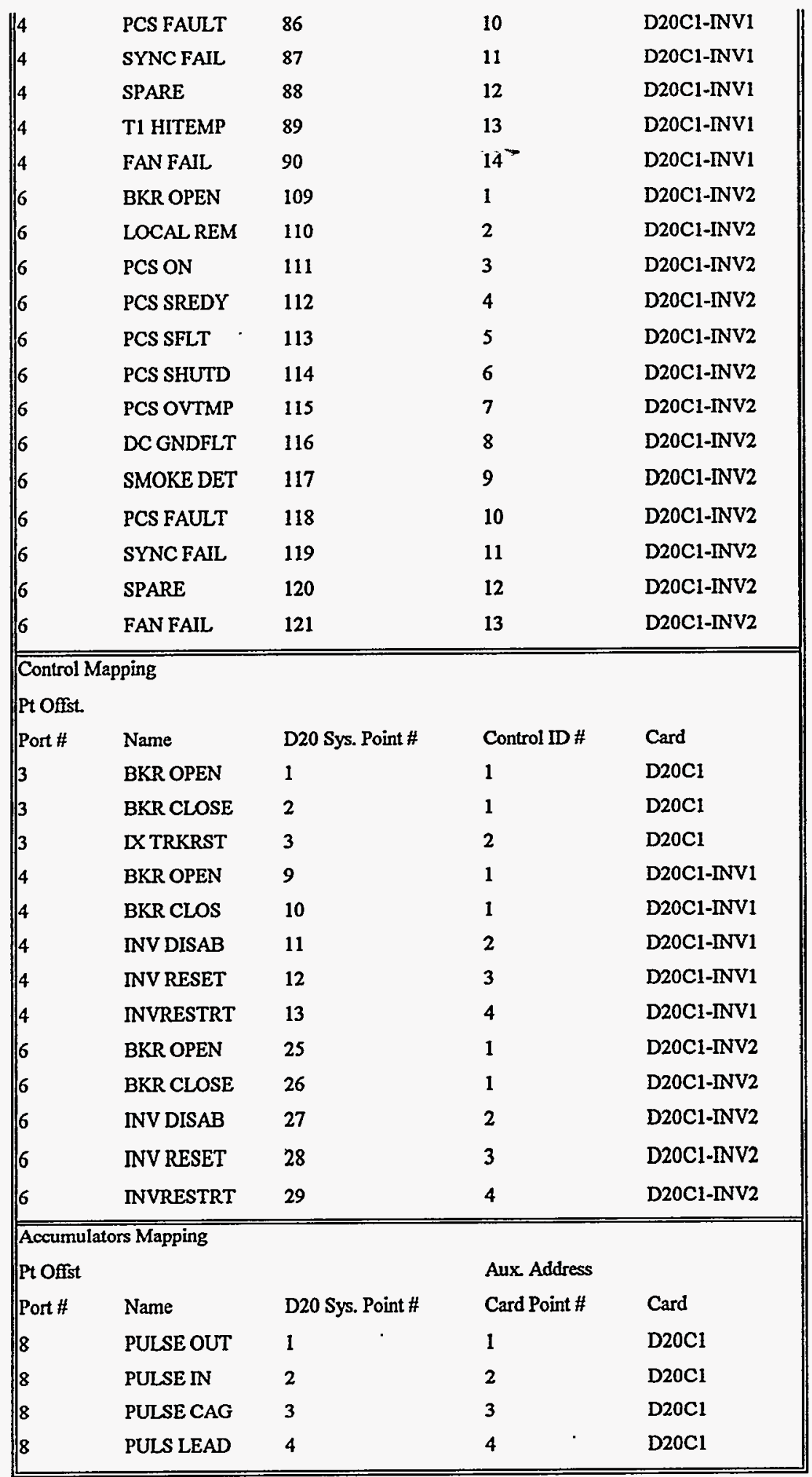


Appendix G

\section{BATCH FILE LISTINGS}




\title{
BATCH FILE LISTINGS
}

\section{G.1. rundaily.bat}

\author{
"Put date into datefile in the format DD-MMM-YY - HH:MM xm \\ date $>3: /$ realflex/data/Kerman.batch/datefile \\ "Use led to parse and convert time string in datefile to the format \\ "MMDDYYkm.csv and put that in the file runl0.bat like this: \\ " cat MMDDYYkm.csv 10minuteaveragefile > MMDDYYkm.csv \\ led 3:/realflex/data/Kerman.batch/datefile $<3: /$ realflex/data/Kerman.batch/ledcmds $>$ \\ 3:/realflex/data/Kerman.batch/errs \\ "Make run10.bat executable. \\ chattr $a=+e$ 3:/realflex/data/Kerman.batch/run10.bat
}

\section{G.2. Example datefile}

24-Nov-93 12:05:00 am

\section{G.3. ledcmds}

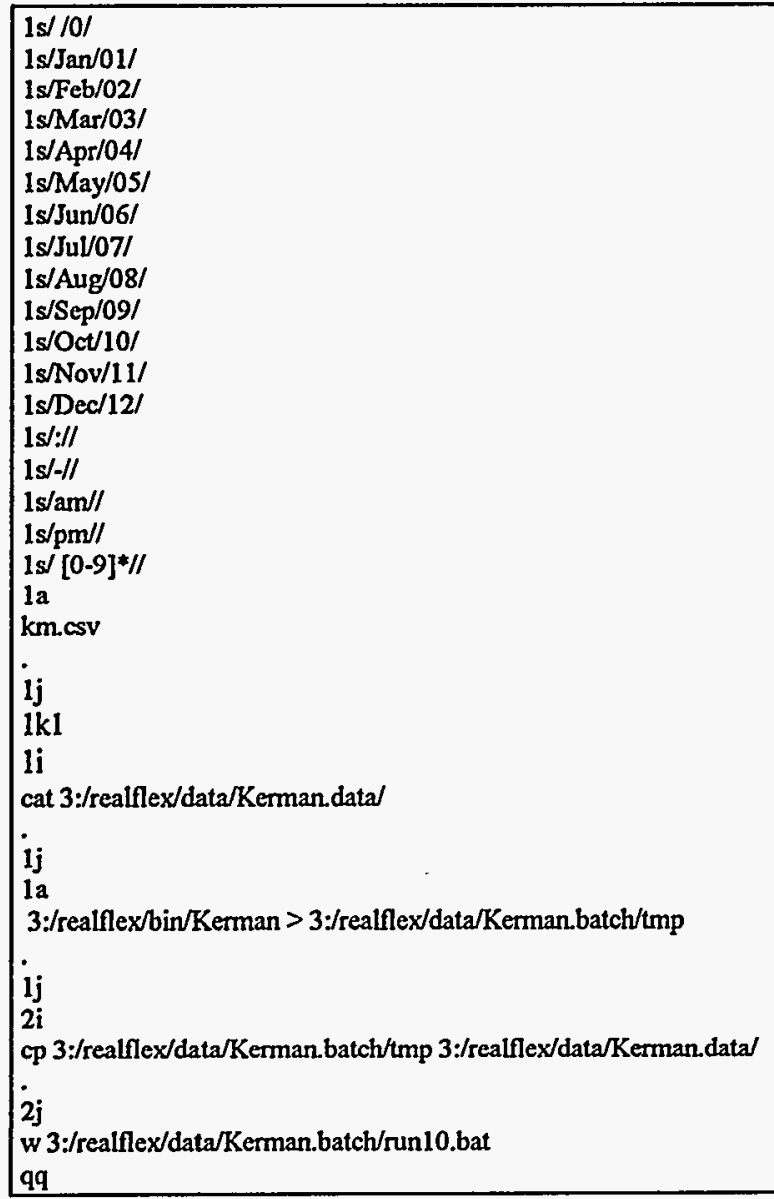




\section{G.4. Example run10.bat}

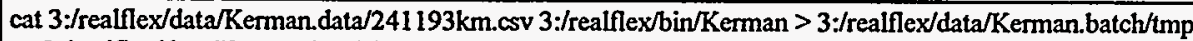

cp 3:/realflex/data/Kerman.batch/tmp 3:/realflex/data/Kerman.data/241 193km.csv

\section{G.5.TDCBACK_INCR}

3:/tdcback/aha \&

files 3:/ $-\mathrm{v}+\mathrm{m} \mathrm{p}=$ ×itmap $\mid$ 3:/tdcback/tdcback aha0 1 continue "save incremental"

3:tdcback/tdc aha0 1 quit

\section{G.6.TDCBACK_FULL}

/tdcback/aha \&

echo "/tmp/dir.report" >/tmp/list

files $3: /-v+d \mid /$ tdcback/tdcdir report $>/$ tmp/dir.report

tddcback/tdcback aha0 1 save $<$ /tmp/list

$\mathrm{rm} / \mathrm{mp} / \mathrm{dir} / \mathrm{report} / \mathrm{tmp} /$ ist

files $3: /-v_{p}=$ bitmap $\mid /$ tdcback/tdcback aha0 1 continue "errors /tdcback/enrors" save verify

Addcback/tdc aha0 1 quit

\section{G.7.TDCBACK_HIST}

tdcback/aha \&

echo "/tmp/dir.report" >/tmp/list

files 3:/realflex/data/hist.save $-v+d \mid$ /ddcback/tdcdir report $>/$ tmp/dir.report

/tdcback/dcback aha0 1 save $</$ tmp/list

$\mathrm{mm} / \mathrm{tmp} / \mathrm{dir}$.report /tmp/list

files 3:/realflex/data/hist.save $-\mathrm{v} p=\wedge$ bitmap $\mid /$ tdcback/tdcback aha 01 continue "errors /tdcback/errors" verbose save verify

$>$ tddcback/verify_log

ttdcback/tde aha0 1 quit

\section{G.8.TDCBACK_CSV}

tdcback/aha \&

files $3: /$ realflex/data/Kerman.data $-v+c p=$ bitmap $/$ /ddcback/tdcback aha0 1 continue "errors/tdcback/errors" verbose save verify $>$ ttdcback/verify_log

/tdcback/tdc aha0 I quit

\section{G.9.TDCBACK_LIST}

tdcback/aha \&

/dcback/tdcback aha $\mathrm{I}$ verbose continue "errors $/$ tdcback/errors" list $>/$ tdcback/list

/tdcback/tde aha0 I quit 
Appendix $\mathrm{H}$

CONTACTS 


\section{CONTACTS}

\begin{tabular}{|l|l|l|l|}
\hline \multicolumn{1}{|c|}{ Item } & Company Responsible & \multicolumn{1}{c|}{ Contact } & \multicolumn{1}{c|}{ Telephone \& FAX } \\
\hline Harris/Westonics Hardware & Harris Corporation & Adam Caballero & $\begin{array}{l}\text { ph: (206) 828-2716 } \\
\text { fx: (206) 828-2149 }\end{array}$ \\
\hline Weather Instrumentation & Augustine + Co. & Jim Augustine & $\begin{array}{l}\text { hm: (510) 525-0464 } \\
\text { fx: (510) 525-9410 }\end{array}$ \\
\hline Database Calibration & Endecon & Paul Hutchinson & $\begin{array}{l}\text { ph: (916) 753-0796 } \\
\text { fx: (916) 753-8469 }\end{array}$ \\
\hline $\begin{array}{l}\text { Database Calibration } \\
\text { Maintenance }\end{array}$ & PG\&E & Dan Divine & ph: (209) 263-7115 \\
\hline R\&D Computer & Endecon & Ben Valega & $\begin{array}{l}\text { ph: (510) 867-3360 } \\
\text { fx: (510) 867-3362 }\end{array}$ \\
\hline General Site Questions & PG\&E & Brian Farmer & $\begin{array}{l}\text { ph: (510) 866-5259 } \\
\text { fx: (510) 866-5447 }\end{array}$ \\
\hline
\end{tabular}

